\title{
The Social Support for International Graduate Students to Obtain Academic Success
}

Ping $\mathrm{He}$

West Virginia University

Follow this and additional works at: https://researchrepository.wvu.edu/etd

\section{Recommended Citation}

$\mathrm{He}$, Ping, "The Social Support for International Graduate Students to Obtain Academic Success" (2013). Graduate Theses, Dissertations, and Problem Reports. 520.

https://researchrepository.wvu.edu/etd/520

This Dissertation is protected by copyright and/or related rights. It has been brought to you by the The Research Repository @ WVU with permission from the rights-holder(s). You are free to use this Dissertation in any way that is permitted by the copyright and related rights legislation that applies to your use. For other uses you must obtain permission from the rights-holder(s) directly, unless additional rights are indicated by a Creative Commons license in the record and/ or on the work itself. This Dissertation has been accepted for inclusion in WVU Graduate Theses, Dissertations, and Problem Reports collection by an authorized administrator of The Research Repository @ WVU.

For more information, please contact researchrepository@mail.wvu.edu. 
The Social Support for International Graduate Students to Obtain Academic Success

Ping He

\begin{abstract}
A dissertation submitted to the
College of Human Resources and Education

at West Virginia University

in partial fulfillment of the requirement for the degree of
\end{abstract}

Doctor of Philosophy in Education
in

Joy Saab, Ed.D., Chair

Paul Chapman, Ph.D.

Sam Stack, Ph.D.

Patricia Obenauf, Ed.D.

Michael Lastinger, Ph.D.

Department of Educational Leadership studies

Morgantown, West Virginia

2012

Keywords: Social support, international graduate students, academic success 


\section{ABSTRACT \\ The Social Support for International Graduate Students to Obtain Academic Success}

\section{Ping He}

Previous studies suggest that international graduate students' academic success is significantly associated with the average grade point (GPA), and this measure is closely related with international graduate students' received academic and financial supports. However, international graduate students' academic success can involve a multidimensional measurement besides the average grade point (GPA). Hence, the current study explored the correlation between international graduate students' perceived social support and perceived academic success from an inclusive perspective. The perceived social support included the categories of academic support, financial support, emotional support and spiritual support. The perceived academic success included the aspects of knowledge learning, research skills, employability, potential earning, professional development and comparative ability. 


\section{Contents}

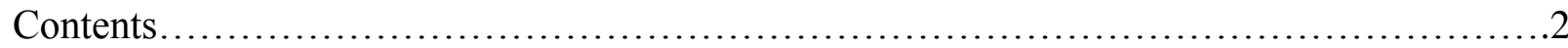

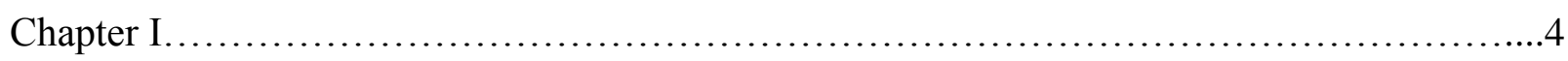

Purpose of the Study....................................................... 10

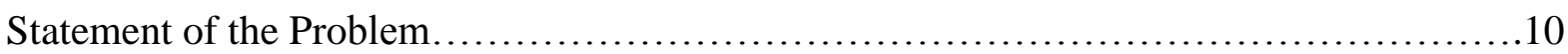

Research Questions..........................................................11

Significance of the Study.................................................... 11

Terms and Definitions......................................................... 12

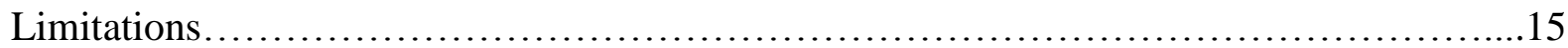

Chapter II................................................................... 16

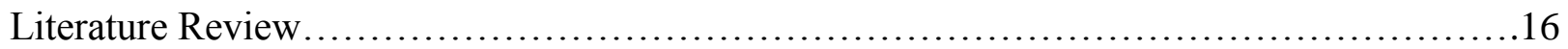

International Graduate Students.................................................. 16

Concept of Social Support..................................................30

Social Support Theories.....................................................39

Academic Success.........................................................57

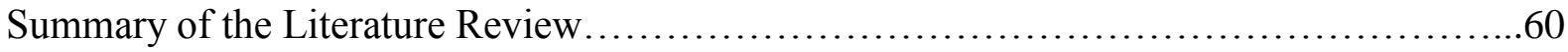

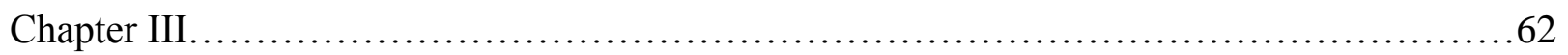

Research Method..............................................................62

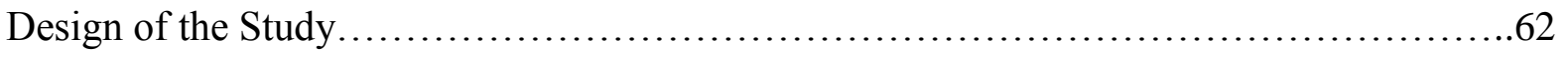

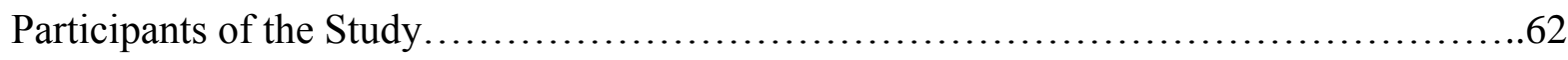

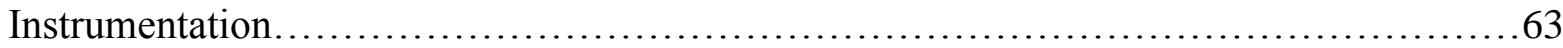

Data Collection..............................................................65

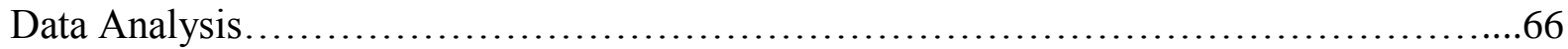




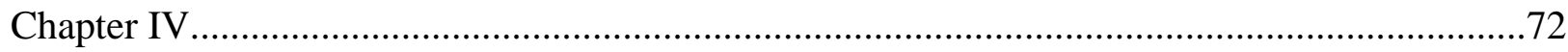

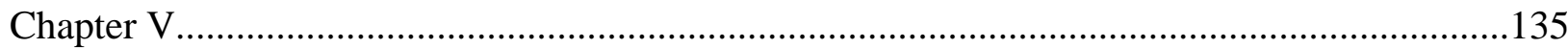

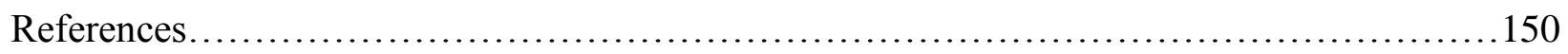

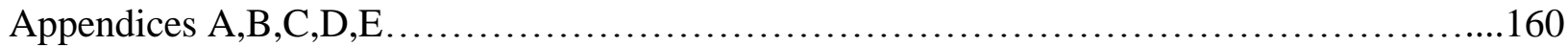

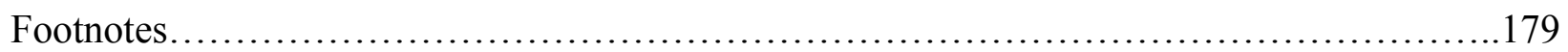




\section{The Social Support for International Graduate Students to Obtain Academic Success}

\section{Chapter I}

\section{Introduction}

As early as the $13^{\text {th }}$ century, the University of Paris already started internationalized education by opening its door to overseas students. Since then, higher educational institutions have realized the importance of internationalized education. Some people believe that the University of Paris has always been the avant-garde of globalizing higher education. Thus they pointed out the necessity of utilizing a common language of instruction like English to meet the academic demands of internationalization of higher education (Altbach, 1998).

In recent decades, colleges and universities have encountered unprecedented challenges created by the forces of globalization. “...the borders of universities have opened in new ways for their services and products..." (Gumport \& Sporn, 1999). The momentum of internationalization of higher education has driven colleges and universities all over the world to make adaptations both in structure and culture in order to catch up with the globalization trend (Bartell, 2003). At the very beginning of twenty-first century, many colleges and universities in North American has started to put internationalization in agenda and even consider internationalization of their curriculum as a strategic priority for the purpose of academic development (Bartell, 2003). Take the recent years for example, "In the 2003-2004 academic year, international students comprised $4.3 \%$ of the total enrollment in U.S. institutions of higher education, accounting for more than 570,000 students (Institute of International Education, 2004)."

With more and more international students flowing into U.S. higher educational institutions, the social problem of international students' adjustment into American society has 
drawn increasing attention of researchers and professionals. Higher educational researchers found out that very few international students would be able to achieve their degrees without encountering any difficulties such as poor socialization with peer domestic students, academic isolation, financial stress, homesickness, role conflicts, spiritual anxiety and even psychological depression and so forth (Mallinckrodt \& Leong, 1992; Rahman \& Rollock, 2004; Swagler \& Ellis, 2003; Yang \& Clum, 1995; Ying, 2005).

However, some researchers have argued that there's actually no difference between the problems encountered by international students and their domestic counterparts, and in the sense of academic adjustment, international students can be regarded actually more as "students" than as "foreigners" (Walton, 1968). These researchers believe that these problems are generally met by both international students and domestic students and they are essentially the same in nature. Nevertheless, most higher educational researchers agree that the problems encountered by international students are unique to this group and thus they require correspondent strategies to deal with these issues in order to complete their studies abroad. According this point of view, distresses international students are experiencing have a significant correlation with the foreign cultural context they are adapting to. Klineberg (1980), Ying and Liese (1991) have also argued that the experienced difficulties of international students originated from their living experiences in the different cultural background in which extra adaptation pressures are exposed to international students compared to their domestic counterparts (Sam, 2001).

International students will first encounter the challenge of cultural adaptation, Furham (2004) illustrated the problems of cultural difference international students may experience when they study abroad. At the moment that they arrive at the host country, international students will immediately meet the problem of language barrier. In the perplexing all-English 
environment, they are distant from family, friends, and other familiar social supports. Usually there's a significant discrepancy between the host culture and their own culture in terms of cultural elements such as attitudes, interpretations, expectations and values. And this difference will inevitably result in negative experiences of confusion, anxiety, stress and so forth. In addition, many international students are constrained to their own cultural group, and thus lack opportunities of communicating and interacting with domestic students. And this will undoubtedly limit their access to more social support resources, thus hinder their acquisition of supportive information in related to their overseas studies (Furham \& Bochner, 1986; Ward \& Kennedy, 1996; Volet \& Ang, 1998; Furham, 2004).

Bochner (1982) has argued that cultural-learning can be an effective media through which international students will be able to successfully adjust to a new cultural environment. In many cases, international students prefer to maintain their own cultural behavior patterns while studying abroad. They are also willing to learn the characteristics of the host cultural system so that they will be able to fit into the new cultural groups for the purpose of achieving their academic success. For short-term learners, such as visiting scholars, keeping their own cultural behavior patterns is particularly important because they don't want to get in trouble when they try to shift back into their own cultural environment. Furnham and Bochner (1986) suggested individuals will successfully adapt to a new cultural environment without experiencing too many distresses through the practice of cultural learning. They also believe that individuals will encounter less cultural anxiety if they acquired more advanced social skills in the new society. In the study of visiting Asian students at Australian universities, Barker et al (1991) found out that emotional support such as friendship, socialization skills training will to a great extent improve international students' adaptation ability (McKinlay, Pattison \& Gross, 1996). 
Some researchers are concerned about the relationship between international graduate students and their professors. Loewenberg (1969) argued there's inequality in the relationship between faculty and students. This may also be applied to the relationship between international graduate students and faculty members. In terms of accessibility of academic resources such as references, academic recording, financial support, faculty members are entitled authority over international graduate students. Professors are powerful and directly influential in international graduate students' studies. International graduate students are usually highly dependent on professors academically and even financially. Some international graduate students have strong self-awareness and adaptive skills. However, in many cases we need to concern about international graduate students' adaptation situation in related to demoralization and attrition (Loewenberg, 1969).

Experiencing discrimination is another problem that international graduate students may face when they study abroad. Perrucci and Hu (1995) did a quantitative study applying regression model considering international graduate students' life satisfaction when they study in American college and universities. They found out that among the 12 predictors, experiencing little or no discrimination is highly correlated with satisfaction. This predictor comes in fourth just after the predictors of being married, having good language skills and having extensive exposure to U.S. culture. This implies that adaptation to a new culture requires multiple and complicated socially supportive elements for international students to obtain their academic success. Perrucci and $\mathrm{Hu}(1995)$ also pointed out that cultural adaptation for international graduate students doesn't necessarily mean that they need to change their own cultural behavior patterns. The host culture also has the responsibility to make changes to integrate international students into the new cultural circumstances. Perrucci and Hu (1995) also argued that 
international students will encounter abundant challenges such as language barrier, cultural conflict, financial anxiety, socialization isolation, emotional stress, spiritual anxiety and so forth. There's a great possibility that international students will not be satisfied with their study abroad. Carr, Koyama and Thiagarajan (2003) designed a research on the values of Asian international women graduate students. They also developed a support group program and a message board for this group to deal with their specific issues. The support group program provided a socializing circumstance for these Asian women international graduate students. They could actually obtain supportive resources from this group and also from the counselors when they are addressing their adaptation issues. They found out that group support is advantageous over individual counseling because group support resources can decrease individuals' feelings of being helpless, isolated. And at the same time, individuals can also develop their sense of belonging within this support group so that they will be able to more effectively address their adaptation issues. This program successfully achieved its goal of relieving these Asian women international graduate students' adaptive stresses, and thus promoted their academic success.

Studies have found out that international graduate students seek for help through utilizing social support resources for various reasons in spite of the fact that there are many support programs available to them. In the study of Frey and Roysircar (2006), they made a comparison between Chinese international graduate students and non-Chinese international graduate students concerning the way of dealing with stresses. They found out that Chinese international graduate students tend to seek information rather than try to deal with their stressful situation independently. They also found out that many South Asian international graduate students prefer to read books or other printed materials to seek help for their health issues. They finally argued 
that international graduate students tend to seek help from their own national group instead of from domestic group (Frey \& Roysircar, 2006).

Frey and Roysircar (2006) further pointed out that for Asian international graduate students increased interaction with domestic graduate students was associated with increased adaptation and decreased stress. However, international graduate students reported a very small proportion of domestic friends compared to their own national group friends. In addition, there's no clear evidence that this is due to the fact that international graduate students' preference to seek social support from their own national group or that domestic students have a low need to make international friends (Frey \& Roysircar, 2006).

Generally, the problems encountered by international graduate students can be categorized into four types: academic problems, emotional problems, financial problems and spiritual problems. It's highly possible for international graduate students to encounter these problems when they study abroad. The situation is probably even worse for this population. Hence, social support seems very important for international graduate students to achieve their academic success. Social support provides a safe social background for international graduate students to confirm their experiences and cope with adaptation problems. Usually, international graduate students obtain supportive information through the informal channels within their own ethnic or national groups. However, research found out that the developed connections between international graduate students and other ethnic groups or between international graduate students and domestic students will relieve the adaptation stresses. Professional counselors found out that it is important to do research on the international graduate students' socialization situation before counseling them. For example, is he or she experiencing language barrier? Is he 
or she experiencing social integration problems and so forth (Carr, Koyama \& Thiagarajan, 2003)?

\section{Purpose of the study}

Therefore, the purpose of this study was to investigate the relationship between the social support perceived by the international graduate students and their academic success in MidAtlantic Land Grant Research University. This study conducted multiple regression analysis and phenomenological research methods to see if there's correlation between social support and academic success for international graduate students at Mid-Atlantic Land Grant Research University. The goal of this study was to help higher educational administrators and researchers to further understand the problem of international graduate students' adjustment in Mid-Atlantic Land Grant Research University so that more measures will be taken to facilitate international graduate students' adjustment to the new learning and living environment in Mid-Atlantic Land Grant Research University.

\section{Statement of the problem}

According to previous studies, over half a million international graduate students are studying in U.S. higher educational institutions nowadays. Rose and Redwood (2010) argued that there is an immediate academic need for higher educational researchers to make further studies of international graduate students' adaptation experiences when they study in U.S. colleges and universities. Meanwhile, many American colleges and universities have realized the important contributions made by international graduate students in promoting cross-cultural interaction for the purpose of keeping pace with the trend of economic and cultural globalization. Many American higher educational institutions have started to build up international students and scholars' office or center through which campus diversity initiatives are promoted by 
organizing various international events or festivals. International graduate students can also seek information related to addressing their concerns and issues when they study in these colleges and universities (Rose-Redwood, 2010).

Undeniably, international graduate students are playing a significant role in enhancing the campus diversity across the United States. These students have become an essential component of the overall multicultural structure of U.S. colleges and universities. Previous studies also found out that the researches about international graduate students usually emphasize the field of cultural adjustment, academic stress, emotional anxiety, life satisfaction and so forth when they study in U.S. colleges and universities. These studies examined international graduate students' overall interpretations of American culture and their learning and adaptation experiences. However, very few studies provide a deep explanation of the framework of social support theories related to international graduate students' academic success, and the inclusive analysis of the correlation between the predictors of social support and international graduate students' academic success while studying at U.S. higher educational institutions (Rose-Redwood, 2010).

\section{Research questions}

To identify and analyze the relationship between social support for international graduate students and their academic success, the following questions were asked:

1. Can we predict international graduate students' GPA from measures of gender, age, region, year, academic, emotional, financial and spiritual supports?

2. Can we predict international graduate students' perceived academic success from measures of gender, age, region, year, academic, emotional, financial and spiritual supports? 
3. How do international graduate students perceive their academic success and social support system at Mid-Atlantic Land Grant Research University?

\section{Significance of the study}

This study will contribute to the body of knowledge in the area of international graduate students, social support and academic success. The study will enlighten higher education institution's administrators in the application of administrative measures to assist international graduate students to achieve their academic success. Form another perspective, this study will also help international graduate students realize and face their current situation and offer information to international graduate students to seek support for obtaining their academic success.

This study will also add to the existing knowledge on academic success of international graduate students by analyzing the correlation between social support and academic success. The regression will also be analyzed to predict the relationships between variables under perceived academic success and perceived social support. The analysis of perceived academic success is significant because the objective measurement of academic success (GPA) is not reliable to measure the relationship between social support and academic success. The subjective measurement of perceived academic success will be categorized into six areas: knowledge learning, research skills, employability, potential earning, professional development and comparative ability. The perceived social support includes academic support, emotional support, financial support and spiritual support.

The current study will also advise higher institution administrators on the effect of their administrative measures to assist a specific and important student group (international graduate 
students) on campus to adjust to the new cultural environment quickly and obtain academic success.

\section{Terms and definitions}

The following terms are defined to assist in understanding the research study:

1. Internationalization- Ellingboe (1998) defined internationalization as “... as the process of integrating an international perspective into a college or university system. It is an ongoing, future-oriented, multidimensional, interdisciplinary, leadership-driven vision that involves many stakeholders working to change the internal dynamics of an institution to respond and adapt appropriately to an increasingly diverse, globally focused, ever-changing external environment".

2. International graduate students- Graduate students who are neither citizens nor permanent residents of the host country.

3. Regions- "The geographic areas of the world (Africa, Asia, Europe/Canada, Latin America, Middle East/India) which produced international graduate students for this study (Feizi, 1990)."

4. Social support- Cohen (2000) has identified three types of social support titled emotional support, informational support and instrumental support. Emotional support refers to supportive resources through which individuals will be able to seek emotional comfort. Informational support refers to supportive resources through which individuals will be able to seek information for problem solving. Instrumental support refers to material or financial supportive resources through which individuals will be able to solve problems.

5. Social integration- Brissette et al. (2000) defined social integration as "participation in a broad range of social relationships. It is a multidimensional construct thought to include a 
behavioral component - active engagement in a wide range of social activities or relationships - and a cognitive component - a sense of communality and identification with one's social roles ."

6. Social network- Smith and Christakis (2008) defined social network as human relationship of " both (a) egocentric (or local) networks, in which an individual is located at the hub of a wheel, with the rim delineating his/her social contacts and the spokes the ties that connect them; and (b) socio-centric (or socio-metric, complete, or global) networks, in which all or nearly all members of a community or group and their linkages to each other are represented."

7. Social identity- Tajfel (1978) defined social identity as "that part of an individual's selfconcept which derives from his knowledge of his membership of a social group (or groups) together with the value and emotional significance attached to that membership" (p. 63).

8. Attachment- Prior and Glaser (2006) believed that attachment means "an affectional bond or tie between an individual and an attachment figure (usually a caregiver). Such bonds may be reciprocal between two adults, but between a child and a caregiver these bonds are based on the child's need for safety, security and protection, paramount in infancy and childhood. The theory proposes that children attach to caregivers instinctively, for the purpose of survival and, ultimately, genetic replication."

9. Academic success- Prevatt et al (2011) argued that it is hard to accurately define academic success. However, they listed a variety of measures that " focus on discrete aspects of academic success in students, such as motivation, career decidedness, academic skills, emotional and psychological factors, and social and interpersonal factors." 
10. Knowledge Obtaining- Refers to the process through which individuals obtain knowledge or information by employing various methods such as observation, imitation, memorizing, presentation, communication, case-studying, modeling, problem-solving and so forth.

11. Research skill- Research skills include skills of critical thinking, problem solving, analysis, dissemination and so forth.

12. Employability- Hind and Moss (2011) defined employability as "In simple terms, employability is about being capable of getting and keeping fulfilling work. More comprehensively, employability is the capability to move self-sufficiently within the labour market to realize potential through sustainable employment. For individuals, employability depends on the knowledge, skills and abilities they possess, the way they use those assets and upresent them to employers, and the context (e.g. personal circumstances and labor market environment) within which they seek work."

13. Potential earning- Potentiality of making money in the future.

14. Professional development- Speck and Knipe (2005) defined professional development as "skills and knowledge attained for both personal development and career advancement. Professional development encompasses all types of facilitated learning opportunities, ranging from college degrees to formal coursework, conferences and informal learning opportunities situated in practice. It has been described as intensive and collaborative, ideally incorporating an evaluative stage."

15. Comparative ability- In this study, comparative ability refers to international graduate students' ability to achieve academic success compared to other international graduate students.

\section{Limitations}


This study was limited to the international graduate students in Mid-Atlantic Land Grant Research University. Hence this study is institutionally specific and the findings of this study may be limitedly applicable to other higher educational institutions.

In addition, this study applied multiple regression models for the quantitative part to analyze the research results. Different statistical methods may produce different interpretation of the research results. 


\section{Chapter II}

\section{Literature Review}

This chapter reviews the concept of social support and international graduate students as they pertain to academic success. More specifically the literature will address: international graduate students' issues; the concept of social support; social support theories and the concept of academic success.

\section{International Graduate Students}

Internationalization of higher education. To understand international graduate students, we must first analyze the phenomenon of internationalization of higher education all over the world. Habu (2000) has identified three motivational factors that have activated the internationalization of higher education. Commercialism is believed to be the first motivational factor that has promoted the globalization of higher education. This motivational factor is due to the fact that many higher educational institutions have integrated market values and imperatives into the academic world. From this perspective, higher educational institutions regard themselves as commercial businesses competing for student resources with each other or at the global level for the purpose of making profits. On the other hand, higher educational institutions consider their students as consumers or customers and they are not necessarily part of the academic community. The problem of this argument is that one of the main characteristics of the academic community, that is students, are separated from this context. The relationship between higher educational institutions and students are commercialized and thus hinder authentic academic interactions between students and the academic institutions.

Habu (2000) further argued that the second motivational factor of internationalization of higher education is associated with the necessity of establishing cross-border academic 
communities for the purpose of promoting free ideas exchanges at the global level. According to this vision, the purpose of academic globalization is to enhance the conversation between the East and West, and thus achieve the goal of establishing a stable global development environment. The conception of this vision has originated from the European enlightenment movement, however, it is indisputable that the demand of exchanging ideas globally is capable of uniting people of different nationalities together and thus creating a global learning community. And this undoubtedly has also inspired American higher educational institutions to actively engage in the activities of internationalization of higher education.

Habu (2000) finally pointed out that promoting diversity is the third motivational factor activating internationalization of higher education. This motivation is rooted in the European Enlightenment movement from which U.S. has developed its Enlightenment ideals. In addition, this vision also has some relation with the post-modernism movement in the western world. Accordingly, we may argue that the academic requirement of establishing a global learning community and the need to enhance diversity have been the most powerful forces driving U.S. higher educational institutions to invest and involve in the internationalization movement. However, unfortunately, the belief in the motivational factor of commercialization has once dominated the higher educational community. Higher educational administrators once believed that colleges and universities are virtually commercial businesses competing for student consumers, and it is applicable to the global level. However, this vision is conflicting with higher educational institutions' aims of pursuing knowledge through cooperative learning and appreciate multicultural diversity in the global learning community. Under this view, the globalization of higher education will be headed to a wrong direction (Habu, 2000). 
International graduate students. International graduate students are an essential outcome of the internationalization of higher education movement. They are playing a significant role in the efforts of achieving U.S. Enlightenment goal of establishing a friendly global learning community and thus promoting the academic diversity on campus. At the personal level, international graduate students travel overseas to pursue knowledge and personal achievement. They contribute importantly to the development of graduate education in U.S. higher educational institutions with regard to academic, cultural and economic aspects. They also serve as the multicultural messengers conveying information through the international academic channels. (Chen, 2007).

Undoubtedly, international graduate students have contributed significantly to promote the cultural diversity at colleges and universities in U.S. In the United States, we have seen the improvement of cultural diversity on most higher educational institutions' campuses in related to the internationalization aspect. Higher educational researchers have realized the importance of developing corresponding policies at the institutional level on behalf of promoting the academic communication and interaction between the international students and domestic students. In practice, many U.S. colleges and universities are putting on the internationalized learning and teaching activities to meet the goal of promoting multicultural diversity on campus. According to Milem (2003) and Chang (2001), there are three elements of campus diversity: structural diversity, diversity-related initiatives and diverse interactions. Undeniably, international graduate students have enriched the three elements of diversity on U.S. college and university campuses.

However, most international graduate students will encounter adjustment problems on their way to achieve academic success. Feizi (1990) has argued that the most common problems international graduate students will encounter will be the difference cultural context, the different 
value systems and the different educational systems. These adjustment problems can be generalized into the following categories: academic problems, emotional problems, financial problems and religious problems.

Academic stress. Previous studies have identified three types of academic stress. The first type is related with social adjustment. According to this vision, academic stress will lead to healthy issues such as anxiety or even depression. The second type of academic stress is defined as a failure of adaptation to the academic situation. In the third interpretation of academic stress, Lazarus and Cohen (1977) argued that academic stress is associated with individuals' cognitive ability through which individuals make cognitive responses to the situational demands. Wan, Chapman and Biggs (1992) also argued that "within a cognitive framework, academic stress occurs when the environmental demands are evaluated as exceeding a student's resources for coping with them."

Lazarus (1976) correlated academic stress with individuals' cognitive level of appraisals. He believed that there are two levels of cognitive appraisals through which individuals make responses to the academic situational demands. The first type is called primary appraisal through which individuals make evaluations of learning experiences in correspondence to the situational demands. The second type is called secondary appraisal through which individuals identify the available supportive resources and employ these resources to deal with the academic stresses (Lazarus, 1976). According to this explanation, we can believe that international graduate students respond to the academic situational demands differently through utilizing their different levels of cognitive appraisals.

International graduate students who study in U.S. colleges and universities may encounter various academic stresses because of the highly challenging educational environment. And this 
may consequently impact international graduate students' learning experiences and thus they will have difficulty understanding and applying their knowledge. Previous studies found out that international graduate students are the group who are frequently attacked by academic stress. Additionally, compared with social and personal problems, problems caused by academic stress last longer and have the strongest negative impact on international graduate students' learning experiences. Therefore, we can believe that academic stress has a significantly negative correlation with international graduate students' learning experience, psychological health, physical health, life satisfaction and evaluation of living experiences in the host country (Wan, Chapman \& Biggs, 1992).

Classroom struggle. Previous studies also found out that for international graduate students, classroom culture is one of the most difficult things to deal with in U.S. educational environment. Most international graduate students have struggled when they adjust to the new learning environment. According to Sarkodie-Mensah (1998), this struggle is due to most international graduate students' completely different learning environment in their home country where professors are regarded as academic authorities. In their country, professors are highly respected and must be addressed with their titles. However, in the U.S. educational environment, the learning atmosphere is very informal. Students are allowed to speak up anytime, have food, and even walk around. Students can also call their professors by the first name. Most international graduate students feel uncomfortable and even dilemmatic in such educational environment. American professors also expect their students to participate actively in the learning activities, and participation usually will be taken into account in students' final grades. However, most international graduate students come from a learning environment in which lecture is the main teaching method. Thus they are used to the corresponding learning strategy of 
listening and taking notes in class instead of expressing their own opinions in class. Thus most international graduate students will have a hard time trying to transfer to a new learning mode (Sarkodie-Mensah, 1998).

Another learning difficulty for international graduate students in the classroom is cooperative learning in groups. For Asian international graduate students, the challenge of group learning is the creativity of individual thinking that is expected to contribute to the group work. Most international graduate students prefer to work with students of their own nationality or other international graduate students. They feel more comfortable to work in this way because they are usually afraid to work with their competitive and aggressive American classmates. They can feel learning empathy in this learning experience and thus rebuild up their learning confidence. However, this way will isolate them from their domestic classmates and block them from benefitting from their domestic learning partners. Another possible embarrassing learning situation is that they don't have international counterparts in the classroom. Thus they don't have other choices and have to work with their domestic classmates. However, in such learning situation, the domestic graduate students usually dominate the whole cooperative learning process. And this will inevitably frustrate international graduate students' learning motivation and efficiency (Sarkodie-Mensah, 1998).

Language barrier. Although most international graduate students have passed the language test TOEFL (Test of English as Foreign Language) before they entered the programs in U.S. colleges or universities, they still have language barriers when they study and live in an all English academic environment. Previous studies found out that language ability will significantly affect the level of international graduate students' level of academic and social adjustment to the new learning environment. English-language proficiency has been found to be importantly 
associated with international graduate students' academic performance and achievement. In White and Brown's (1983) study on international graduate students, they found out that one of the most important obstacles of international graduate students' academic performance is English-language barrier. They also found out English-language ability is significantly associated with the level of international graduate students' life satisfaction and learning experiences in the host country (Lee, Abd-Ella, \& Burks, 1981; Fletcher \& Stren, 1989).

Perrucci and $\mathrm{Hu}(1195)$ have also argued that international graduate students' level of social adaptation to the host society is importantly dependent on their English-language skills. International graduate students are forced to learn and understand the new norms, values and expectations system in the dominant culture on behalf of achieving their academic success. In this process, English-language barrier will undoubtedly impede their effective adaptation to the new social behaving patterns. Consequently, the mal-adaptation to the new society will lead to the possibility of international graduate students' poor academic performance and achievement. Therefore, it is of essential importance for higher educational institutions in U.S. to effectively respond to international graduate students' adaptation difficulties and employ appropriately corresponding measures or policies with regard to dealing with these problems (Perrucci \& Hu, 1995).

Culture shock. Fernandez (1988) has defined culture shock as "all those beliefs and expectations about how people should speak and act; the social structure and organization; the relationship and rules that govern kinship systems; one's ethnicity; one's socioeconomic status; and the ideals, customs, and learned behavior that have become second nature to a person". Under the situation of experiencing culture shock, an individual will feel lost in the foreign cultural background and thus feelings of being left out and disoriented will be created. These 
feelings will negatively influence international graduate students' adaptation responses to the new environment in which they find out that their previous stress-dealing strategies are no longer applicable in the new society. However, it usually takes a while for international graduate students to adjust to the new social behaving system. On the other hand, there is an urgent academic demand for international graduate students to quickly and effectively fit into the new cultural background. Thus international graduate students need to deal with extra stressful issues compared to domestic graduate students who find out that graduate study itself has been very stress provoking. As a result, for international graduate students, it's more likely to undergo negative adaptation experiences which consequently lead to mental anxiety and so forth when they are encountering cultural discrepancies (Dao, Lee \& Huang, 2006).

Cultural adjustment. Nearly all international graduate students need to address the problem of cultural adjustment in order to achieve academic success. Kagan and Cohen (1990) have argued that acculturation and assimilation must be considered when we discuss cultural adjustment. Although we don’t have specific definitions of acculturation, assimilation and adjustment, these terms are frequently applied in the studies of behavior, value and attitude changes with regard to mental health and social integration.

Cultural adjustment occurs when one starts living in a foreign cultural background. Individuals also start to interact with the members of the new society. Previous researchers have developed and designed many cultural adjustment models which include "behavioral", "personal" and "social network elements". Goldlust and Richmond (1974) developed one of the earliest cultural adjustment models. Their model investigated the elements of "internalization", “identification", "value and attitude change”, and "pre-migration influences”. Olmedo (1979) developed a cultural adjustment model called "full measurement model". This model measured 
individuals" "linguistic", "psychological" and "social-cultural" changes when they are placed in a foreign cultural background. Rosenthal and Hrynevich (1985) also developed a comprehensive cultural adjustment model which examined individuals" "ethnic identity" when they live in a foreign culture. Their model included the discussion of the elements of "language", "religion", “social activity", "maintenance of cultural traditions", "family life" and "physical characteristics" (Kagan \& Cohen, 1990).

These models are basically applicable to the situation of international graduate students with regard to cultural adjustment. For example, international graduate students may experience "cultural resistance" when they study abroad. Inevitably, there will be significant disparity between international graduate students' home culture and the host culture. Many international graduate students tend to keep their own cultural behaving traditions in the new system. Thus they feel uncomfortable to adapt their behavioral patterns in order to fit into the new cultural context. In this process, "cultural resistance" occurs which will subsequently lead to international graduate students' cultural adaptive issues such as hesitation, anxiety, distress, depression and so forth. Another example may involve international graduate students' group or ethnic identity when they study abroad. International graduate students prefer to associate with people of their own ethnicity. However, social integration issues may occur to international graduate students in this process. They may isolate themselves from the major cultural community. Consequently, they may run up against cultural barriers to achieve the goal of academic success.

Social marginalization. McClure (2007) has argued that international graduate students may experience social marginalization as a result of the failure of cultural adjustment to the new cultural community. Many international graduate students will go through the period of feeling confused and disoriented when they start living in a new society. This discomfort contributes 
significantly to international graduate students' negative experiences such as feelings of being lonely, helpless, isolated, marginalized and so forth. Before going further to discuss the implication of social marginalization to international graduate students, we need to introduce the definition of social marginalization.

McClure (2007) stated that "social marginalization is, firstly, a feeling of a sense of loss and deprivation concerning all that is familiar, particularly home, family, friends and culture. Secondly, it is the experience of being isolated from or feeling rejected by members of the new social and academic culture. Thirdly, it is the psychological reaction to cultural differences ranging from stress to indignation. Finally, it is the experience of constraints and sacrifice concerning one's future choice due to traditional gender roles”. For example, international graduate students may find it difficult to make domestic friends because of the cultural difference. It may take a long time for international graduate students to feel accepted by the domestic major cultural community.

Emotional sustenance. Previous studies found out that friendship contributes importantly to international graduate students' success in cultural adaptation to the new cultural environment. Most international graduate students have a strong need to make friends concerning dealing with negative experiences such as feeling lonely, homesick, helpless and so forth. Researchers also found out that a stable friendship network will provide effective supporting resources for international graduate students to reduce negative feelings. Thus this will also help international graduate students to maintain a healthy physical and psychological being for the sake of achieving academic success. Accordingly, the absence of a supportive friendship network will hinder internal graduate students' successful academic performance (Storti, 1990). 
Previous studies have identified three types of friendship: the domestic friends, other nationality friends and the same nationality friends. The domestic friends are an important informational resource for international graduate students to attain information about the major cultural and linguistic learning. Other nationality friends provide a supplemental social resource for international graduate students to enrich their socialization experiences. Finally, the same nationality friends provide international graduate students with an essential support to establish their group identity. Researchers found out that socialization with domestic students is significantly associated with international graduate students' level of satisfaction. Unfortunately, most international graduate students tend to make much more home cultural contacts than host cultural interactions. Although it is undeniable that the home cultural group will support international graduate students' adaptive efforts, it is also inevitable that in the long run this preference will negatively impact international graduate students' adaptive health. For example, international graduate students who constrain their socialization in the home cultural group will frequently communicate in their own language. This will hinder the improvement of their English-language skills. Thus researchers have argued that "the multicultural bond offers the opportunity to learn about new cultures and to develop cross-cultural competence, leading to improved career prospects and arguably greater social cohesion both locally and globally" (Huntington, 2004; Brown, 2009).

Financial anxiety. Although, most international graduate students have obtained various financial supports from their departments or colleges, for example, teaching assistantship or research assistantship, the income from their assistantship can only meet their very basic expenses. For instance, the average annual income from assistantship for the international graduate students in WVU (Mid-Atlantic Land Grant Research University, U.S.) is around \$ 
11,000 to $\$ 15,000$. This is far below the poverty line income in America which is around $\$$

27,000 . We can believe that international graduate students are actually one of the poorest groups of people who live in U.S.

Although most international graduate students have tuition waiver, there may be anxieties about "hidden costs". For example, in WVU international graduate students have to pay special fees (including PRT transportation, library service, rec-center service and so forth). Additionally, this fee has been increasing very fast. For example, for the time period from 2008 to 2012, special fee has doubled from around \$ 400 in 2008 to \$ 800 in 2012. However, their income from assistantship still remains the same. At the same time, they are not allowed to work more than 20 hours a week. This means they are not qualified to apply for most of the off- campus jobs. The assistantship income is their only money for living. Thus, most international graduate students have to spend a lot of time concerning about their financial issues. Unfortunately most institutions choose to ignore such individual concerns. There's even some worse situation for this concern in which international graduate students frequently encounter structural difficulties related with their financial issues created by the institutional staff (Harris, 1995).

Financial anxiety has become one of many international graduate students' major impediments on their way to achieve academic success. Take Chinese international graduate students for example. In Chinese culture, there's a fundamental common belief that grown-up children are supposed to support their old parents. Many Chinese international graduate students have received financial support from their parents for their undergraduate study. Thus they are expected to provide similar support to their family after they are financially independent. Therefore, many Chinese international graduate students' scholarship or assistantship income not only supports their own living expenses, but also contributes to their family expenses. This 
undoubtedly has added weights to Chinese international graduate students' financial burden on their shoulder. Thus Harris (1995) has argued that "this sense of obligation is fundamental in Chinese culture, where the western individualized 'ego' is culturally alien and, in more traditional families, the notion of 'I' and 'family' as distinct entities are without meaning'.

Religious identity. International graduate students are very diverse in religion, and this often puts them in a dilemmatic situation in related with their religious practice. Take Muslim international graduate students as an example, actually they are a big proportion of the international graduate students population in U.S. Brown (2009) argued that Muslim people only appreciate their own faith thus many of them are obstructed from opportunities to openly communicate with diverse informational resources. However, people from other countries may share the same religion, for example, Christianity, thus these people can build up bonds through practicing their same religious activities. However, previous studies found out that there are barely religious interactions between Muslim people and outside people from other religions (Brown, 2009). Thus Muslin international graduate students are very restricted to their own religious group, and to a big extent isolated from other national or international groups both spiritually and emotionally.

Christian, Hindu and Buddhist international graduate students can also encounter this dilemma when they practice their religion. This is even true for atheist international graduate students. Take Chinese international graduate students for example, most of them are atheists when they first come to study in U.S. They felt bound to families and friends and had a strong sense of security in emotion when they were in China. However, everything has dramatically changed at the moment they arrived in U.S. They had to seek new emotional bond and figured out that religion is actually an effective way here to make new friends. However, since they 
didn't grow up in a religious cultural background, it's very hard for them to make transformation in faith. Most of them feel very lost and are to a certain degree struggling in this dilemmatic situation.

Researchers also argued that people understand and appreciate life through religious activities from which they will be able to find the meaning of life. However, can we actually believe that Muslim international graduate students can seek more comfort from their religion than students from other nationalities and religions? It can be a challenge for Muslim international graduate students to keep their religious identity in a different cultural background. When they practice their religion in their homeland, their religious identity will come naturally. However, if they live in a different society, even an open one like U.S., they may need to fight for and claim their religious identity. No matter how religiously friendly a society is, there may be intangible discriminative power influencing people's attitudes towards an extremely exclusive religion (Brown, 2009).

There have been many studies focused on the contribution of religion to the meaning and quality of life. In Fife, Adegoke, Mccoy \& Brewer's (2011) research, they reviewed different studies and found out that people's life satisfaction was significantly associated with the meaning and purpose of life which people can find through religion. They also found out that however for younger people who are seeking meaning and purpose of life through different activities, life satisfaction is less related with religion. They pointed out that younger people pay more attention to their social identity than religious identity such as romantic relationship, work relationship, family relationship etc. They are more interested in improving their productivity and creativity in related with the overall social activities through which they will be able to find the meaning and purpose of their life. However, for older people, they tend to be more religiously dependent for 
the purpose of seeking and appreciating the meaning of life. Thus there's a tendency that older people are more satisfied with their life than younger people. In the previous studies related with the quality of life, very few cases were focused on the sample of older people and religion. Thus there's a need for researchers to include younger people and more diverse populations in related with the study of religion. And there're even fewer studies concerning international graduate students' religious issues and identity (Fife, Adegoke, Mccoy \& Brewer, 2011).

\section{Concept of Social Support}

In the Encyclopedia of Applied Psychology, Social support (Social Support, 2004) is defined as "information that leads individuals to believe that they are valued, respected and loved and that helps them to cope with major life stressors and the challenges of everyday life. Social support is a special kind of social interaction that can appear in different ways and that can be both a psychological and a tangible resource provided by a social network, for example, by friends, family members or colleagues".

According to the Encyclopedia of Applied Psychology (Social Support, 2004), people’s physical and mental health is strongly related with social support through which people are accessible to supportive resources and thus associated with the group and society. Social support has positively affected people's well-being at the very early stage of human history. Researchers have long been realizing the importance of social support contributing to people's healthy life. However, the theoretical and pragmatic implication of this field still needs to be explored and conceptualized on behalf of improving the quality of people's life. "During the past 30 years or so of research, social support has become one of the central variables in many fields of psychology, epidemiology, public health and education. It seems to be a potent variable for 
helping to explain the beneficial or detrimental effects of other people being around an individual who is coping with the challenges of daily life or major life stressors (Social support, 2004)."

Sociological researchers have defined "social support" in many different ways. However, they generally agree that "social support is a group of family, friends, neighbors and colleagues who are available all the time for psychological, physical and financial support (Liebler \& Sandefur, 1998)". They also confirm the important contribution of social support system to people's physical and psychological health by improving people's adaptive ability and coping strategies. Social integration and social network are essential factors affecting the availability of social support resources. Individuals build up supportive social relationships through social integration. Also individuals perform social interactions in the established social networks for the purpose of being socially accepted and integrated into the society. On the contrary, individuals who are isolated from necessary social support resources will not be able to deal with stressful situations effectively and thus develop negative feelings such as confusion, anxiety, stress, depression and so forth. The findings of social support studies have not only been applied to the psychological area, but also many other social areas such as education, business, politics and so forth. Researchers believe that individuals' physical and mental well-being is significantly associated with the availability of stable social support resources (Al-Jenaibi, 2011).

In Lakey and Cohen's (2000) studies of social support, the major theory is about the correlation between stress coping strategies and social support. They argued that individuals' failure to effectively deal with external stresses will result in negative emotional situations. Conversely, an effectively established social support system around individuals will decrease individuals negative feelings and thus enhance individuals' adaptive abilities and coping strategies. 
In another study of college students' problem-focused coping strategies, Chao (2011) found out that there's a significant positive relationship between social support and the decrease of mental problems. The researcher also found out that students who actively look for social support resources from their families or friends tend to be able to more effectively cope with their stressful situations than their counterparts who lack access to necessary social supports. The quality and quantity of available social support is also significantly related with the degree to which individuals effectively resolve their stress issues. The low quality and amount of social support resources will not be capable of improving individuals' negative emotional situations. Previous studies also found out that low level of quality and quantity of social support will negatively impact individuals' physical and mental well-being. Individuals accessible to social support resource low in quality and quantity tend to be involved in more unhealthy activities including abuse of drugs, alcohol, sleep and diet disorder, and so forth. More seriously, the low quality and quantity of social support may even lead to individuals' deteriorating behaviors such as dissatisfaction and hatred of life, and even suicide. Thus, we can believe that the level of the quality and quantity of social support will decide the extent to which individuals get rid of life stressors and maintain well-being. This is also applicable to international graduate students' stress-coping situations. International graduate students who are exposed to sufficient social support resources with high level of quality and quantity will be able to achieve a balance between life stress and well-being. On the contrary, those with low social support will lose the balance and this may consequently lead to these international graduate students' detrimental life situations. Thus, we can believe that social support can effectively promote international graduate students' stress-coping and adaptive ability when they adjust their behaving patterns to the host society (Chao, 2011). The disconnection between social support resources available to 
international graduate students and their perceived life stress will inevitably lead to negative impact on well-being and mal-adaptive behaviors associated with their academic failures.

Maulik, Eaton and Bradshaw (2010) argued that social support is strongly associated with the maturity of individual's social networks through which one's well-being will be fully developed and maintained. In addition, they also believed that social support is relatively of more qualitative characteristics. Through the application of social support resources, individuals cope with life stresses and achieve personal and social development. Thus the conceptualization of social support has been developed in a cultural background of social network and is inextricably related with it.

Maulik, Eaton and Bradshaw (2010) also discussed the functionality of social support in their study. They suggested two functions of social support. One is termed as "stress-reduction function, and the other is as "referral function". They believed that social support is capable of buffering individuals' life stresses by providing individuals with supportive consults and other assistances. Through utilizing various social support resources including both tangible and intangible resources, individuals will be able to attain empathy and understanding from others, and develop effective stress-coping strategies, and thus reduce the negative impact of life stresses. Previous studies also found out that the level of social support utilization is significantly related with the reduction in the usage of health services. Maulik, Eaton and Bradshaw (2010) also offered a specific example of the "stress-reduction" function of social support. They argued that this function of social support will be clearly displayed in the situation of unexpectedly arising stresses. Under such condition, all involved aspects of individuals' available social support resources will be activated, leading to the effect of "stress-buffering" during this process ( Maulik, Eaton \& Bradshaw, 2010). 
The other function of social support, termed as "referral function" has been demonstrated in both positive and negative aspects, depending on the different way that individuals respond to life stresses. If individuals are actively engaged in the application of various social support resources and positively assess the effect of social supportive assistances, individuals may be able to efficiently decrease life stresses. Conversely, if individuals negatively evaluate social support resources and thus resist assistances, individuals may not be able to deal with life stresses effectively ( Maulik, Eaton \& Bradshaw, 2010).

According to Ozkahraman et al. (2011), social support is defined as "interpersonal transactions that involve the expression of positive effect, the affirmation or endorsement of the person's belief or values, and the provision of aid or assistance". Individuals' mental well-being can be well maintained through the application of social support resources. The possible negative impact of life events can be blocked away from individuals' life experiences if social support resources are employed appropriately. Previous researchers found out that under extremely stressful conditions, for example, going through a divorce, losing a job and so forth, individuals will experience less distress with the assistance of social support resources. In the case of international graduate students, it is not arguable that international graduate students are dealing with a highly stressful situation in which they are not only required to commit their academic responsibilities, but also involve in the complicated process of cultural adaptation. During this process, social support seems essentially important to help international graduate students improve stress-coping strategies and adaptive abilities in a new society. Accordingly, higher educational institutions with international graduate students should provide sufficient social support resources to help them identify problems and develop strategies to deal with life stresses. Recent studies of social support for international graduate students have realized the significant 
correlation between the effective application of various social support resources and academic performance and life satisfaction. However, the realization of the theoretical implication of social support doesn't necessarily mean the effective practice of this theory in the pragmatic sense (Ozkahraman et al., 2011).

Other researchers have studied social support from different perspectives. For example, Wilks and Spivey (2010) studied the relationship between social support and resilience. They argued that social support functions as "stress-buffering" through its relationship with individuals' behaviors through which individuals' characteristics such as "stress", "anxiety", "self-efficacy" will be measured effectively. Resilience is one of the most important features of effective stress-coping strategies. They also believed that the quality of being resilient of individuals' behaving patterns will be built up if individuals successfully coped with previous stresses. The quality of resilience in one's stress-coping behaving system means that an individual has successfully adapted in the stressful situation and it also implies that this specific individual has obtained the capability to deal with future stresses. Wilks and Spivey (2010) further noted that the quality of resilience is a necessity for individuals to successfully develop effective stress-coping strategies and survive the harsh situation. The researchers argued that in the case of international graduate students, social support can promote the quality of resilience in international graduate students' stress-coping behaving system and help them go through the stressful period of their academic life. However, they also pointed out that the accurate mechanism of the production of resilience through social support resources still needs further exploration. One thing is certain that under stressful situations, the sufficient availability of social support resource will be able to promote individuals' stress-coping strategy development and thus enhance individuals' stress-coping resilience. 
Other researchers studied the resources of social support. For example, in the study of Rosenthal et al. (2011), they found out that "perceived social support from close friends and family members" is an essential available social support resource for individuals to deal with life stresses. However, for international graduate students lacking support from family members, they argued that support from close friends is a very import factor for them to handle academic challenges. They also stressed that the academic department and institution can be another important provider of social support for international graduate students. For example, a supportive mentoring relationship and a friendly academic environment can help international graduate students develop sense of belonging in the academia. The researchers finally pointed out that "social support resources from both within and outside the academic environment may serve as important socializing agents in determining which fields of study to pursue, and how to balance multiple demands of life and academics over time (Rosenthal et al., 2011)”.

Baqutayan (2011) researched the relationship between students' academic stress and social support. The researcher believed that it's very possible for individuals to develop psychological issues in a long period of continuous experiences of stress. Even if individuals have developed a responsive strategy to deal with the stress, there's still possibility that this strategy is not capable of relieving individuals' stresses. The researcher also pointed out that the conceptions of stress and coping strategy have been mistakenly understood by people. People usually believe that the concept of stress is negative, and the concept of coping strategy is positive. However, the researcher argued that the situation is much more complicated than this. Previous studies have found out that both stress and coping strategy can be conversely displayed, being either negative or positive depended on individuals' different psychological situations. Thus, in the case of international graduate students, it is important to help them decrease the 
negative impact of stress and ineffective coping strategies and take advantage of the positive impact of stress and effective coping strategies. The researcher further argued that there's a big discrepancy in the effect of stress management between international graduate students who are accessible to sufficient social support resources and those who are socially isolated. In her study, the researcher found out that international graduate students who reported more social support availability reported distinguishingly less academic stress in their adaptation process.

The social support model developed by Cohen and Mckay (1984) provided a multidimensional perspective to examine the correlation between individuals' stress-coping strategies and the social support resources available to them. The researchers reviewed previous studies of social support which have generalized social support into two types of "psychological" and "non-psychological" support. They argued that "psychological" support can be further divided into "appraisal" support and "emotional" support which focuses on the provision of intangible knowledge or information to individuals who are dealing with stressful situations. "Non-psychological" support can also be termed as "tangible" support which focuses on the provision of material support to individuals in a stressful situation. Therefore, they believed that the categorization of social support should be put into three types including "tangible support", “appraisal support" and "emotional support".

Cohen and McKay (1984) further pointed out that "tangible support" seems less effective than "psychological support" including "appraisal support" and "emotional support" in the process of "stress-buffering" for individuals. The researchers then offered an example of this argument. They contended that it seems more likely for individuals from poor families to feel more stressful than those from rich families when they are coping with similar situations. This implies that the effect of "tangible support" on "stress-buffering" may differ among individuals 
from different social backgrounds. Thus it may be unnecessary to distinguish between material support and non-material support. Accordingly, "tangible support" resources have been merely considered in the studies of social support effect on individuals' stress-coping situations.

Cohen and McKay (1984) further argued that the experience of psychological stress is more subjective than objective depended on the situation in which individuals evaluate stress as harmful or harmless. Individuals' "psychological structure" and the stress stimulus' "cognitive feature" are essential in the process in which individuals decide the "stimulus presentation" as threatening or non-threatening. If an individual regards the stress stimulus as threatening, he will employ responsive coping strategies to deal with the situation. Thus it is important for individuals to evaluate the stress stimulus first and then apply appropriate stress-coping strategies in the process of determining and handling stress experiences (Cohen \& McKay, 1984).

The researchers also believed that the evaluation of one's stress stimulus is to some extent dependent on one's interactions with other individuals. They stressed that social support is the provision of information within an interactive social network in which individuals develop the positive feelings of respect, love, responsibility, belongingness and so forth through various interactive activities. The researchers also pointed out that the mechanism of "appraisal support" focuses on external assessment, on the contrary the mechanism of "emotional support" focuses on internal evaluations such as individual's personal feelings and so forth (Cohen \& Mckay, 1984).

Finally, Cohen and Mckay (1984) pointed out that the "emotional support mechanism" can function as "stress-buffering" predictor under the condition that individuals believe that their positive feelings and evaluations have been reduced by stresses. They also believed that social support resources have the capacity of hindering these reductions in individuals' emotional 
system. The studies of the effect of social support resources should involve both individuals' physical and psychological evaluations (Cohen \& McKay, 1984).

\section{Social Support Theories}

After the discussion of the conceptualization of social support, we need to explore the theoretical orientation of social support theories. This will basically relate to social identity theory, social integration theory, social network theory and attachment theory.

Social identity theory. Henri Tajfel and John Turner (1979) were the first developers of social identity theory. They argued that individuals identify other people as "in-group" or "outgroup" members through three important psychological stages. These three stages include "categorization", "adoption" and "comparison". In the first stage, individuals put themselves and other people into different categories according to various standards such as race, region, religion, gender, profession and so forth. Through this way, individuals interact with their social environment and develop the sense of belongingness. During this process, different social norms are employed by individuals inside the same group to make a decision if one's behavior is appropriate or not and make responses to such behavior. However, appropriate behavior in one group doesn't necessarily meet behavior standards in another group. In the second stage, individuals understand and internalize the social norms developed by their social group they belong to. Take Muslim people for example, they first identify themselves as this group members, then they will realize that it is important to conform to social norms in this group. For example, women are supposed to wear veil and cover their body up in public places. During this process, individuals will develop their sense of "self-esteem" and a bounded relationship with group members. In the final stage, individuals tend to make comparison between "in-group" and "out-group" members. And this is often the origination of social bias and discrimination, and 
even social conflict. The reason is that social individuals have the need to maintain their social "self-esteem" and group identity between different social groups. However, quite frequently, there's difference or even controversy in the understanding of social norms and values between different groups. Thus social groups with conflicting social norms tend to compete and fight for their social identities in order to survive in the culturally competitive society (Mcleod, 2008).

Trepte (2006) argued that individuals achieve their social identification through establishing the social status of their own group's norms and values and at the same time challenging and limiting other groups' norms and values. Thus individuals develop their "selfesteem" and "self-enhancement" during this process. The researcher believed that individuals tend to categorize themselves into one or two social groups and discriminate against other social groups whose norms and values they believe rival and threaten their own. The consequence of this prejudice will be the extinction of minority or weak group cultures and the tendency of homogeneity of the cultural variances. And this in the long term will hurt the overall development of the social culture. In addition, social identity theory emphasizes the process of social identification from the perspective of a social group instead of a specific individual in that social group. In other words, social identity theory stresses the importance of collectivity rather than individuality during this process. Tajfel and Turner (1979) argued that "a social group consists of a number of people who feel and perceive themselves as belonging to this group and who are said to be in the group by others". This implies that the interpersonal interactions among social group members occur on behalf of the group benefits as a whole. Additionally, it is not necessary for "in-group" members to understand and accept the beliefs and values of "outgroup" members for the purpose of establishing a social group. Tajfel and Turner (1979) also proposed four fundamental components of social identity theory including "social 
categorization", "social comparison", "social identity" and "self-esteem" based on their definition of three stages of individuals' social identity process. They believed that individuals develop their social identification through the understanding, evaluating and emotionally associating with their social memberships with other social group members in the group through applying the four fundamental principles of social identity theory.

Other researchers focused on understanding the implications of the function of social identification. For example, Ashforth and Mael (1989) argued that social identity theory emphasizes the classification of individuals according to various characteristics. Individuals prefer to define their classification based on their related features such as age, gender, education, religion and so forth and put themselves and other people into different social groups. This implies that it is highly likely that individuals will belong to multiple social groups according to different "categorization schemas", which have been developed on the foundation of individuals" generalized typical characteristics. Ashforth and Mael (1989) further pointed out that there are two fundamental functions of social identification. The first function is related with the structure and classification of the society. Through social identification, individuals are not only able to define themselves, but also able to obtain socially practical understandings of other people. At the same time, the society is divided into different social categories. However, the characteristics of this social classification may change over time, and this will result in the restructure of the social classification according the social identity theory.

Ashforth and Mael (1989) further argued that the second function of social identification is related with individuals' development of self-esteem or self-location in the society. Through social identification, individuals will be able to find their location in the society. The researchers contended that the development of individuals' self-esteem or self-location in the society is not 
only depended on the individualistic characteristics, but also the obvious group characteristics which individuals locate themselves in.

Chen and $\mathrm{Li}$ (2009) argued that the understanding of "intergroup discrimination" is the main part of social identity theory. They also believed that there are three fundamental components of social identity theory including categorization, identification and comparison. During the process of categorization, individuals identify the similarities with other people, and put themselves into the social groups they believe they belong to. This is a process in which individuals define and label themselves and other people in their social group. As a result, individuals' self-concept will be derived from the social group they locate themselves in. Chen and Li (2009) pointed out individuals tend to easily categorize themselves and other people around them. During the process of identification, the researchers believed that individuals make social connections with in-group people, and distinguish themselves from out-group people. This is a process in which individuals make social meanings of themselves and other people. During the process of comparison, individuals further understand the difference between in-group and out-group people, and thus develop a discrimination against people outside their social categories.

Chen and $\mathrm{Li}$ (2009) also believed that one of the important implications of social identity theory is that individuals find meanings of themselves through the social activities in their group. At the moment individuals locate themselves in a social group, they start to develop their selfesteem in this group. Chen and Li (2009) also pointed out that individuals' behavior will be shaped by their group identification. Thus individuals tend to interact with people inside their group and outside their group in different ways. Previous studies found out that individuals develop favoritism toward in-group members and discrimination against out-group members. 
They also prefer to "create in-group enhancement in ways that favor the in-group at the expense of the out-group". For example, in an interesting experiment carried out by some previous researchers, they found out that participants who were randomly put in different groups awarded more points to people they identified as in-group members (Chen \& Li, 2009).

Social integration theory. According to Hardy (2006), social integration is an essential socializing process in which individuals make interactions and connections with each other. He further argued that individuals will benefit from the process of their social integration both physically and psychologically when they believe that they are accepted by their society. Conversely, individuals lacking the sense of social integration will develop negative feelings such as anxiety, depression, isolation and so forth.

Hardy (2006) also believed that persistent negative feelings of depression and isolation will lead to serious health problems such as mental, physical illness and even suicide. He cited the result of the study of the Common Cold study (Cohen, 2004) at Carnegie Mellon University with Children's Hospital and the University of Pittsburg School of Medicine, and pointed out that the decreasing rates of illness was significantly associated with one's increasing social network. Individuals realize their social value through the perception of their social roles such as parent, friend, coworker and so forth, and this will help them develop positive physical and psychological conditions. The researchers believed that positive self-evaluation of individuals' social integration will stimulate the production of hormones to promote one's immune function. The researchers finally pointed out that individuals' multiple social roles are significantly related with the development of positive feelings and healthy situations (Hardy, 2006).

Cohen (2004) defined social integration as "participation in a broad range of social relationships". The researcher argued that both "behavioral component" and "cognitive 
component" should be included in the discussion of individuals' social integration process. The "behavioral component" refers to "active engagement in a wide range of social activities or relationships", and the "cognitive component" refers to "a sense of communality and identification with one's social roles". The researcher also believed that individuals' behaving patterns are developed in a society with widely accepted social norms. A stable social structure is beneficial to the development of individuals' behavior regulations.

Berkman et al. (2000) analyzed the result of one of the most important works on social integration. That is Durkheim's (1951) Suicide. He argued that Durkheim (1951) successfully examined the relationship between individual pathology and the function of social dynamics. He believed that the development of individuals' behaving patterns is based on social facts rather than individual psychological foundations. He pointed out that there's a significant correlation between the changes of social facts and individuals' behavior patterning changes such as the tendency toward suicide. The researcher further argued that, according to Durkheim (1951) who observed social groups with stable suicide rates, the cause of suicide is significantly associated with the level of social integration in that society (Berkman et al., 2000).

Besides Durkheim's systematic theory of social integration, which focused on explaining how social dynamics impact individual pathology, there are many other theorists who interpreted social integration. Blau (1960) developed a tentative theory of social integration in which he focused on individuals' social integration process in their social groups. The researcher believed that other people's consideration and evaluation is essential in determining one's integration into a social group. If people in that social group decide that a person is qualified to be accepted as their group member, this individual will successfully achieve his social integration into this specific social group. Blau (1960) further argued that "the central sociological problem is what 
social processes are set in motion as every member of the group seeks to become or remain attractive to the others". During the process of social integration, individuals need to keep frequent interaction with other group members and thus maintain their attractive status in this specific social group. As a result, this group's social cohesiveness strongly depends upon its members' social interaction and social integration maintenance.

Blau (1960) further pointed out that it's very important for individuals to keep attractive to other group members in order to maintain their status in this group. Individuals with a high social status seem easier to keep themselves attractive than those with a low social status. In addition, individuals who have similar values with other group members are more likely to be accepted and integrated into the specific social group. Thirdly, individuals who are able to meet other group members' social needs through interactive activities are more appreciated and valued by other group members in this group. Simply put, it is important for individuals to develop characteristics that will be empathized and valued by other group members so that they will be accepted and integrated into that social group. Nevertheless, what is also important is that individuals need to identify and regulate their personal qualities that will fit into that social group. (Blau, 1960).

Social network theory. According to Berkman et al. (2000), the concept of social networks has been developed to meet the demands of interpreting individuals' social behaviors such as working behaving, family activities, political activities and so forth. And this interpretation has been based on a network of social connections and relationships. Social network theories have played a significant role in analyzing social relationships beyond the limitation of specific social groups. 
Berkman et al. (2000) further argued that the focus of social network theories is on the collective characteristics of the whole social systematic actors instead of the individual characteristics of individual actors in that social system. And also social network theories will analyze the influence of group activities on individual behaviors. These researchers believed that "network analysis focuses on the structure and composition of the network, and the contents or specific resources which flow through those networks. Social network analysis includes analysis of both egocentric networks with an individual at the center as well as entire networks of networks at the level of communities or workplaces (Berkman et al. 2000)".

Berkman et al. (2000) further pointed out that social network theory has reasonably assumed that individuals' behaving patterns are decided and shaped by the social network system which distributes social resources to individuals and thus encourage or constrain individuals' behaviors. The social network theories have shared many similar assumptions with functionalist theories. For example, they both agree that the structure of a social group determines how social resources will be allocated to individuals and thus influences and shapes individuals' emotions and behaviors. Social network theorists also believed that the actual social network system may not be necessarily built up on the foundation of geographic or kinship connections (Berkman et al., 2000).

Other social network theorists have focused on the research of social network from a different perspective. For example, Smith and Christakis (2008) examined interpersonal relationships privileged by social networks. They believed that social connections have the function of promoting social interactions by providing individuals with information and other social resources. In addition, they pointed out that social relationships are an essential component of social capital that is capable of enhancing social productivities. Previous studies have defined 
social network as a structural social system that is composed of various individual social contacts reported by individuals. Smith and Christakis (2008) further argued that "helpfulness in this context is generally construed as the perceived ability or willingness to provide instrumental support (financial support or aid with practical tasks), informational support, appraisal support (help in evaluating options and making decisions) or emotional support”.

Smith and Christakis (2008) also believed that there's both similarity and difference between social support studies and social network studies. Similarly, both studies emphasize the importance of social interactions among individuals in a society. However, social network studies focus on the social connections around an individual and also analyze the nature of the social connections. For instance, social network researchers may focus on the study of the features of a social network rather than individuals themselves. Therefore, we can believe that social network researchers study the quality of individuals' social connections and also the influence of these social connections on individuals' experiences.

Smith and Christakis (2008) also pointed out that social network theories are based on two types of studies including "ego-centric study" and "socio-centric study". They argued that ego-centric study focuses on social networks "in which an individual is located at the hub of a wheel, with the rim delineating his or her social contacts and the spokes that connect them; and socio-centric networks, in which all or nearly all members of a community or group and their linkages to each other are represented". They believed that the main difference between these two studies is that the former only focuses on social connections that are directly pointed to individuals while the latter includes social connections that are both directly and indirectly associated with individuals. As a result, social network researchers who are doing ego-centric studies will be able to put an individual's social network together by analyzing his social 
contacts information. However, socio-centric network researchers need to observe and analyze the information from both individuals and their social context in order to complete their social network studies (Smith \& Christakis, 2008).

Salvini (2010) argued that the emphasis of social network theories is to analyze the impact of social networks on individual behaviors. He also believed that this influence is measurable with regard to the verification of the ways that social networks affect individual behaving patterns. The researchers further pointed out that one of the most important achievements of social network theories is the decreased limitation of previous social network studies that have been described as techniques instead of theories. In spite of the fact that social network researchers nowadays are still highly dependent on various technical methods such as statistic data collection, data analysis, graph visualization and so forth, they also have improved social network studies from a pure technical level to a research level through generalizing and conceptualizing their research result and developed "social network theories".

Salvini (2010) also pointed out that previous researchers have offered a definition of social network studies as the researches of the structural social patterning and its influence. Social network researchers believe that the structure of a society is formed by basic social units, that is, social activity performers or actors and the relationship between these performers or actors. The performers or actors include all social units, ranging from individual level to national level, or even international level. And the relationships between these performers or actors refer to situations that are controlling, depending or cooperative through the allocation of social resources. The major goal of social network studies is to analyze these situations in which individual behaviors are restricted or promoted. Social network researchers study the characteristics of social relationships through which social resources are distributed to different 
levels of the social system. They argued that the understanding of individual performers or actors and the understanding of social relationships are mutually complementary and beneficial to one another's development (Salvini, 2010).

The researcher further pointed out that the logical order of social network studies is from macroscopic level to microscopic level, that is from the whole social structure to specific individual behavior. Social network researchers strongly oppose the separation of social relations and individual behaviors. Social network theories have derived from studies that are multidimensional and that focus on how structural social patterns affect individual behaviors through various social connections or relations (Salvini, 2010).

Finally, Salvini (2010) also stressed that the behavioral interaction patterns should be the focus of social network studies. He argued that to a great extent individual behaving patterns are shaped by the larger social system he lives in. On the other hand, many social network researchers contend that the combination of individual behaving culture in a society is essential to determine societal success or failure. They believe that social network studies include two fundamental research principles involved in the study of individual behaviors. One is that social network research is guided by formal mathematical theories; the other is that social network research is performed through collecting and analyzing empirical data (Salvini, 2010).

Attachment theory. Bowlby (1969) has proposed the basic ideas of attachment theory. He argued that "the attached figure, most often but not necessarily the mother, creates a secure base from which an infant or toddler can explore and venture forth". He believed that attachment is an essential component of individuals' behaving system. The attachment mechanism has the similar function of other human beings' biological system, such as blood circulation and so forth. The attachment mechanism provides individuals with a stable psychological environment in 
which individuals will be able to keep the metabolic stability. The researcher also pointed out that individuals' attachment formation starts from one's childhood and goes further into adulthood, and builds up a stable social foundation for individuals' later social activities. He argued that the establishment of a stable attachment mechanism in individuals will enable them to constantly maintain a healthy and supportive connection with the larger social group they live in (Berkman et al., 2000).

Bowlby (1969) further pointed out marriage can be an important attachment mechanism for adult individuals. Stable marriage is capable of providing individuals with a secure psychological environment from which individuals will be able to explore the world. The researcher also believed that through attachment mechanism individuals will be satisfied for the need of feeling being loved and respected, and will also develop the sense of security and reliability. Subsequently, this will help individuals build up his self-esteem which is essential for individuals to develop a healthy and promoting connection with the external social system (Berkman et al. 2000).

Bresnahan and Mitroff (2007) argued that attachment theory has provided us with a theoretical framework to understand how childhood attachment mechanism affects adult behaviors. They pointed out that attachment theory indicates that individuals' attachment mechanism formed during the early stage of one's life has a significant and lasting influence on adult living experiences. That is to say, individuals internalize the interpersonal relationships during his early life period and apply this mechanism to deal with social issues he encounters in his adult life. Nowadays, attachment theorists are still exploring the field of adult behavior related with childhood influence such as the development of security, trust, cognitive behavior and so forth. Bresnahan and Mitroff (2007) believed that the internalized attachment mechanism 
is essential for individuals to understand themselves and other people in and outside their social group and thus help them establish self-esteem and promote interactive activities with other people (Bresnahan \& Mitroff, 2007).

Connors (2011) argued that the formation of an attachment mechanism through the intimate bonding relationship between infant and caregiver is essential to individuals' later adult development. The establishment of an attachment relationship needs to meet specific standards. First, there's a stable and important emotional connection between two particular individuals including the care-receiver and the caregiver. Second, the care-receiver persistently seeks help from the caregiver. Third, the bonding relationship is an emotional foundation through which individuals will be able to develop the sense of security and further explore and deal with stressful life situations. Connors (2011) also pointed out that the purpose of establishing an attachment relationship is for individuals to develop the sense of security. Thus the quality of the caregiver being accessible and responsive is of great importance for individuals to maintain his attachment mechanism. Undeniably, the attachment mechanism contributes significantly to human being's meaningful living parallel to other important biological mechanism of human being.

Connors (2011) also believed that attachment theory implies that individuals increasingly learn how to regulate emotional and social issues through attachment mechanism that is formed in the early stage of their life. During this process, individuals gradually build up self-esteem and recognize the importance of social connections with other people. The researcher further argued that the function of attachment mechanism extends to individuals' adult life and significantly influence their later social behaving patterns. The successful development of secure sense during 
individuals' childhood will help them build up the sense of trust in other people in their later adult life.

Connors (2011) also stressed the importance of the further exploration of adult individuals' psychological condition of attachment process. However, he also pointed out the potential difficulty that attachment researchers may meet when they do researches on adult individuals. For example, adult individuals may be asked to cooperate with the researcher and express their experiences related with attachment relationships. This may pose problems for attachment researchers. Nevertheless, the researcher found out that adult individuals will feel more comfortable to express their intimate relationship issues if they are assured of an autonomous situation. Conversely, adult individuals who lack the sense of security will report vague memory of their childhood experiences and respond briefly to the assertion of normal development experiences. These adult individuals are reported to lack a mature internal attachment mechanism, thus they show dysfunctional symptoms in their later adult life when they deal with complicated social issues. For example, they feel confused, angry, depressed in a socially stressful situation. Connors (2011) finally argued that "disorganized or unresolved adults appear to remain significantly affected by unresolved loss or trauma and show lapses in reasoning and collaborative discourse ".

Other attachment researchers including Riggs et al. (2002) also argued that attachment theory focuses on studying the development of individuals' attachment mechanism, or in another term "individuals' internal working model", which has been formed in the relatively early stage of one's life and continues its significant influence on later adult behaviors. They believed that individual's attachment mechanism affects adult behaviors especially in stressful and emergent situations throughout one's life despite of the fact that this mechanism is usually developed in 
one's early years of life. Attachment researchers have unanimously agreed that individuals in early life stage such as childhood, teenage constantly interact with their caregivers or particular individuals they have an intimate relationship with. Through this interactive process, individuals will develop understanding of themselves and other people and thus establish a profoundly influential "internal working model” throughout their adult life. Some attachment researchers also argued that since the influence of attachment mechanism is especially obvious in stressful situations, there should be an important correlation between individuals' attachment behavior and the social support seeking response. Nevertheless, the researchers found out that individuals will make sure that other people are reliable and trustworthy support-providing resources before they make decision to display their attachment behaving responses in front of life stresses, and such responses appear differently at individuals' different attachment stages (Riggs et al., 2002). Riggs et al. (2002) also discussed the definition of adult attachment. They argued that there are two types of adult attachment including "secure attachment strategies" and "insecure attachment strategies". In most cases, during the process of individuals' physical and psychological development from childhood, adolescence to adulthood, the "secure attachment strategies" will also be developed and function as stress buffers through individuals' interactive activities with their friends, family members and other social support resources. Individuals with developed "secure attachment strategies" are capable of understanding and interpreting the self realistically and establishing healthy social relationships. In addition, individuals with developed "secure attachment strategies" tend to openly communicate with people and interpret external information more realistically and objectively. Individuals with developed "secure attachment strategies" also tend to show their attachment needs when they are in stressful situations and this will subsequently decrease the risk of mental problems. For example, compared with individuals 
with "insecure attachment strategies", individuals with "secure attachment strategies" seem to more frequently take advantage of various professional counseling resources when they are experiencing life stresses that go beyond their stress-coping capabilities (Riggs et al., 2002).

On the contrary, individuals with "insecure attachment strategies" will not be able to deal with stressful situations effectively and tend to be more likely to develop various mental problems. The researchers further argued that it also seems more likely for individuals with "insecure attachment strategies" to develop an unrealistic image of the self and other people and thus result in the cognitive disorder inside these individuals. And this may destructively impact individuals' current and future behavior. Consequently, individuals with "insecure attachment strategies" will find it very difficult to establish trustworthy interpersonal relationships. And in extremely stressful situations, they are not willing to seek assistance from various social support resources. Their insecure internal mechanisms will be reinforced by the occurrence of negative life events in their later life (Riggs et al., 2002).

Social support theory. Cohen (1985) is one of the most representative social support theorists. He has defined the concept of social support and categorized social support into esteem support, informational support and instrumental support. He has also developed complete social support models that interpreted the cause and occurrence of stress-buffering effects. These models were named main effect model and stress-buffering model.

Cohen (1985) defined esteem support as "information that a person is esteemed and accepted". He argued that self-esteem will be developed and promoted if individuals maintain constant communication with other people and feel accepted by other people. There are also other alternative terms for esteem support such as emotional support, expressive support, selfesteem support, ventilation and close support and so forth. Cohen (1985) defined informational 
support as "help in defining, understanding and coping with problematic events. It has also been called advice, appraisal support and cognitive guidance". He believed that interpersonal relationship is an essential component of this type of social support. Individuals develop various social connections through daily activities. Those connections will help them relieve life stresses and develop positive internal working models. This type of social support can also be termed as diffuse support and belongingness. Finally, Cohen (1985) defined instrumental support as "the provision of financial aid, material resources and needed services”. Instrumental support can be provided to individuals by giving time, materials or financial aids to individuals who are in a stressful life situation. This type of social support can be also termed as material support and tangible support (Cohen, 1985).

According to Cohen (1985), the first type of social support model, that is the main effect model of social support, functions in the way of benefiting individuals generally in the condition that individuals are accessible to stable social support resources and positive interpersonal experiences in the social networks. In the main effect model, social support is believed to be a main influential factor that is significantly associated with individuals' overall well-being by providing individuals with a stable and predictable life situation. In this model, social integration is also regarded as an important element that may help individuals reduce various negative social experiences that contribute importantly to mental problems. Cohen (1985) believed that this type of social support views individuals' social role as significant aspect of individuals' social adaptability such as "regularized social interaction" and "embeddedness" and so forth. And also this type of social support will help individuals develop a healthy psychological environment through social interaction, social integration, relational rewards and status support ect. 
Cohen (1985) further pointed out that in the second type of social support model, that is stress-buffering model, individuals' define the term of stress differently, and the way of this definition may affect individuals' helplessness experiences and the development of self-esteem. Individuals experience helplessness because individuals lack the confidentiality to deal with stressful situations effectively. Individuals may also fail in establishing self-esteem because they attribute the unsuccessful coping with stresses to their internal capabilities or personality.

As it is shown in figure 1, Cohen (1985) has interpreted the social support mechanism of stress buffering. He believed that social support will significantly affect the possibility that stress is associated with illness. He argued that social support may function as the mediator between individuals' encountering with a stressful event and his response. This means that individuals exposed to supportive social resources will be able to deal with stressful situations more effectively. He perceives that various social support resources will be available to him, and he has confidence in his own stress-coping abilities. Thus he evaluates the stressful situation realistically and is ready to deal with it. Cohen (1985) further pointed out that "adequate support may intervene between the experience of stress and the onset of the pathological outcome by reducing or eliminating the stress reaction or by directly influencing physiological processes". Social support has the capability of relieving negative impact of life stress on individuals. For example, with the assistance of social support, individuals may perceive the problems less seriously, or individuals may be less sensitive and responsive to stressful situations, or individuals may engage in healthier stress-coping behaviors and so forth (Cohen, 1985). Figure 2.1 Social Support and the Buffering Hypothesis 


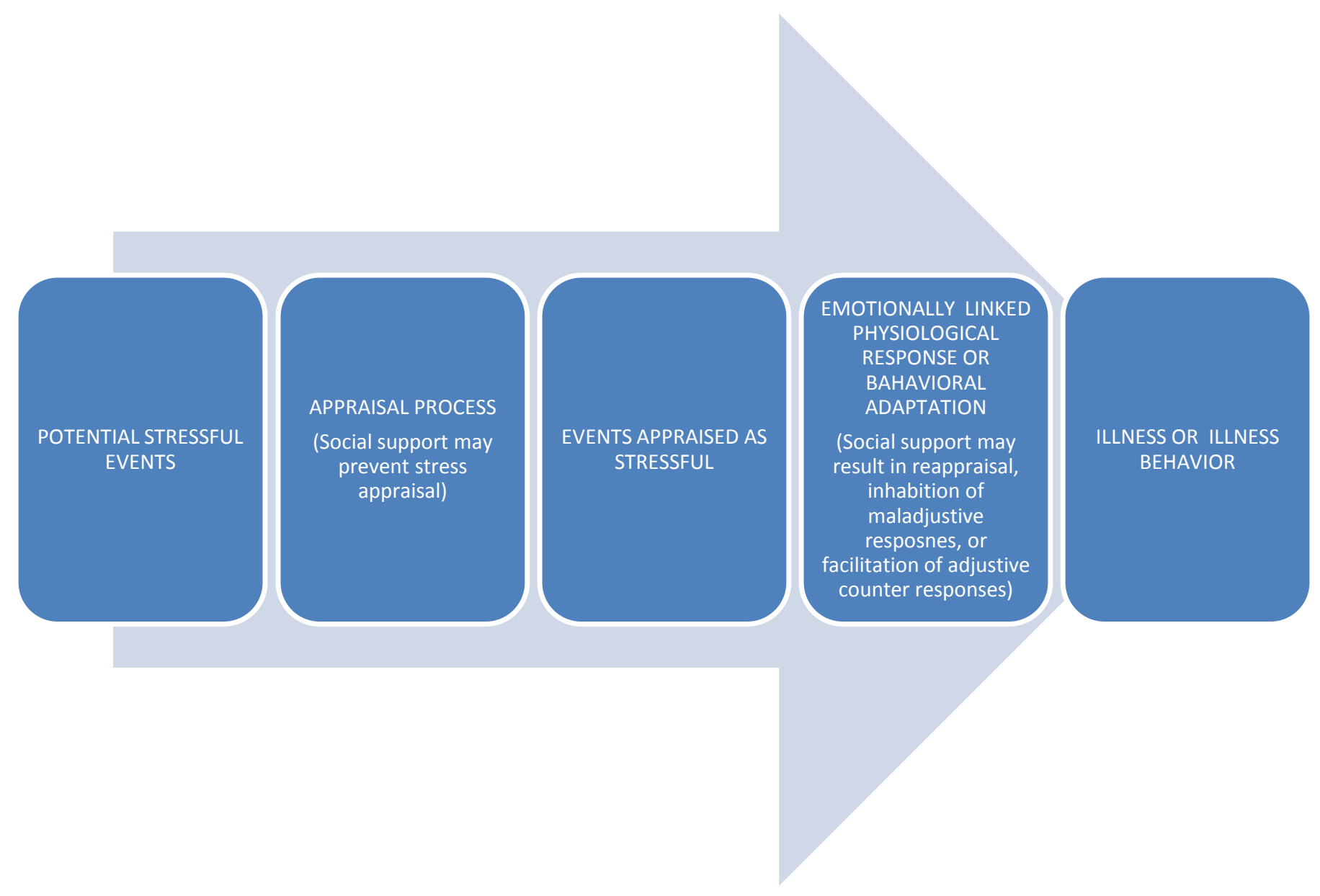

Note: Two points at which social support may interfere with the hypothesized casual link between stressful events and illness. Adapted from "Social Support Measurement and Intervention: A guide for health and social scientists" by Sheldon Cohen, Lynn G. Underwood and Benjamin H. Gottlieb, 2000, Copy right 2000 by Oxford ; New York : Oxford University Press. Adapted with permission (see footnote 1).

\section{Academic Success}


Researchers have found out that it is difficult to make an accurate definition of academic success, especially in graduate study. Nevertheless, educational researchers and higher educational institutions agree that two measurements contribute importantly to graduate students' academic success including GPA (grade point average) and completion of graduate degree. These two measures have been frequently used to assess graduate students' academic success. Previous researchers believed that these two measures evaluate different aspects of graduate students' academic success (Wimberley, McCloud \& Flinn, 1992 ). However only GPA cannot necessarily predict a graduate student's academic success which is an inclusive concept involving the elements of knowledge learning, research skills, employability, potential earning, professional development, comparative ability and so forth.

Previous researchers, for example, Hlebec, Kogovsek \& Ferligoj (2011) also studied the potential predictors of graduate students' academic success. They reviewed the educational persistence models and motivational theories which performed meta-analysis of previous studies concerning graduate students' academic success. In these studies, nine categories were included to predict graduate students' academic success including achievement motivation, academic goals, institutional commitment, perceived social support, social involvement, academic selfefficacy, general self-concept, academic-related skills and contextual influences (including financial support, size of institution and institutional selectivity). They found out that none of these categories were reciprocal to academic success. However, they considered GPA as the main measure of academic success. They also found out that among all these predictors, academic self-efficacy and achievement motivation were most significantly associated with graduate students' GPA. This means that these two factors were the best predictors for GPA. 
However, the rest seven factors were not significantly associated with graduate students' academic success (Hlebec, Kogovsek \& Ferligoj, 2011 ).

Nathan Kuncel and Sarah Hezlett (2007) used standardized test such as GRE (Graduate Record Examination), GMAT (Graduate Management Admissions Test) and so forth as the predictors of graduate students' academic success. They believed that these standardized tests effectively predict graduate students' subsequent academic performance during their graduate study. However, they also pointed out that these standardized predictors are not capable of evaluating more subjective elements of graduate students' academic performance such as learning motivation or research interest which are essential for graduate students to maintain and complete their graduate study. They argued that more reliable measures of graduate students' academic success must be developed for future research such as knowledge learning, research skills, employability, potential earning, professional development, comparative ability and so forth.

Nathan Kuncel and Sarah Hezlett (2007) further argued that it is very important to explore the "actual nature" of graduate students' academic performance. By discussing the "actual nature", they referred to graduate students more subjective aspects of academic performance as we have mentioned above. For example, graduate students' obtaining of knowledge, research skills (ability of research and publication), employability, administrative performance and so forth. They pointed out that previous studies have examined important outcomes of graduate students' academic performance but they have barely involved these aspects that are significantly related with graduate students' academic success. Thus it is necessary for us to develop an inclusive measurement of graduate students' academic performance for future research. 
This inclusive measurement of graduate students' academic success will effectively assess the essential characteristics of a successful graduate student, including international graduate students. It is true that the definition of "academic success", especially for the group of international graduate students, is hard to make. However, there's no doubt that GPA is only one aspect of this multidimensional measurement. International graduate students need to do much more than a good GPA in order to achieve academic success. For example, successful international graduate students must have the ability of performing effective self-regulated learning in order to build up a strong knowledge background for their research. International graduate students also need to effectively practice interpersonal communicative skills in a foreign language learning community. They should have a complicated and practical researchrelated skill capacity including qualitative and quantitative research skills, computer and technical skills, foreign languages, historical knowledge, global awareness and so forth. They should be capable of collaboratively working in a multicultural academic environment. They also need to develop leadership for the purpose of managing their research projects through to completion, and take responsibility to make decisions during this process with strong selfmotivation and participating desirability.

International graduate students' academic success is perceived from a multidimensional perspective involving complicated social elements. However, it is agreeable that successful international graduate students have learned to "effectively balance the social and academic aspects of their graduate study, expect to succeed, and may be described as social proficient, goal oriented, and intrinsically motivated" (Ellis \& Worthington, 1994; Scheuermann, 2000). Previous studies also found out that academically successful international graduate students tend 
to be more employable, have higher self-esteem (Filozof, Albertin \& Jones, 1998) and pose fewer problems to the host society.

\section{Summary of the Literature Review}

This chapter has reviewed literature on: internationalization of higher education, international graduate students, concept of social support, social identity theory, social integration theory, social network theory, attachment theory, social support theory and concept of academic success. More specifically, the social support theories have been broadly reviewed. The literature also has reviewed the studies of international graduate students' specific social adjustment issues.

The literature has provided a theoretical framework for the analysis of the correlation between international graduate students' perceived social support (as independent variable) and perceived academic success (as dependent variable) in this research. The five related social support theories (social identity theory, social integration theory, social network theory, attachment and social support theory) have been systematically outlined for the purpose of establishing a supportive theoretical foundation for the quantitative study of the social support for international graduate students to obtain academic success in this research. 


\section{Chapter III}

\section{Research Method}

Five main sections have been discussed in this chapter including Research design, participants in this study, instrumentation, collection of data and data analysis. The goal of this study was to provide a research concerning international graduate students' perceived social support and perceived academic success. The instrument designed by the researcher was applied: International Graduate Students Social Support Survey (IGSSSS) (see appendix C). This instrument contains three parts: Demographic Information, Perceived Social Support and Perceived Academic Success. Interview questions were designed according to research question three for the qualitative part of this study.

\section{Design of the Study}

A mixed methodology was applied in this study and it provided both descriptive and inferential analysis to investigate how international graduate students perceive their academic success and social support system in Mid-Atlantic Land Grant Research University and identify if there is a correlation between international graduate students' social support and academic success. This study is composed of qualitative and quantitative researches. For the qualitative part, the phenomenological research method was conducted to analyze international graduate students' lived experiences of academic success and social support when they study in MidAtlantic Land Grant Research University. The researcher interviewed 4 individual international graduate students ( 2 females and 2 males) from five areas (South-East Asia, Middle-East, Africa, Europe and Latin-America) to collect the descriptive data about international graduate students' study abroad experiences related with their academic success and social support. For the quantitative part, the demographic factors (including gender, age, region, year) and the perceived 
social support categories (including academic support, emotional support, financial support and spiritual support) are the independent variables. GPA and the perceived academic success are the dependent variables. The statistical data were collected through the application of the structured questionnaires in the instrument (IGSSSS). This instrument were delivered to international graduate students in Mid-Atlantic Land Grant Research University (WVU) to evaluate their perception of the social support as measured by the second part of this survey (Perceived Social Support) and the academic success as measured by the third part of this survey (Perceived Academic Success). A demographic section precedes these two parts (Perceived Social Support and Perceived Academic Success) of this survey to identify the gender, age, region, academic year, GPA information of the participants.

The phenomenological research method has been chosen for the qualitative research part of this study because the researcher wants to know how international graduate student perceive their academic success and social support system when they study in Mid-Atlantic Land Grant Research University. According to Lester (1999), the phenomenological research method aims to interpret individuals' (or actors') lived experiences in a situation and how individuals (or actors) perceive those experiences. In this specific study, the individuals (or actors) were the international graduate students in Mid-Atlantic Land Grant Research University. The situation was the international graduate students' living experiences in WVU. Lester (1999) also argued that the purpose of phenomenological research method is to collect important information about how individuals perceive their living experiences. The method to apply phenomenological research is qualitative including interviews, discussions, and participant observations. The finding result will be represented from the perspective of the research participants. For this study, individual interviews were conducted to collect information about international graduate 
students' perception of academic success and social support system when they study in WVU.

The finding result for the qualitative part was represented from the perspective of the international graduate students participated in this study.

\section{Participants of the study}

The population of this study was the international graduate students in Mid-Atlantic Land Grant Research University (WVU). This university has been considered because it has an attractive academic diversity on campus. It is offering nearly 200 degree programs including undergraduate and graduate levels. It has an international student population (currently 1300 international students) from 109 countries all over the world (http://oiss.wvu.edu, 2012). Thus it has provided a reliable research sample for this study.

In addition, there's a professional organization named the Office of International Students and Scholars (OISS) in this university to provide important information about international graduate students for this study. This organization is playing an essential role in helping international graduate students successfully adjust to the new learning and living environment. It offers assistance to international graduate students "by providing social, cultural and educational programs, and by answering questions and giving advice about personal concerns, cultural differences, immigration regulations, and financial matters" (http://oiss.wvu.edu, 2012). This research was performed with the assistance of this office.

100 international graduate students from five regions (South-East Asia, Middle-East, Europe, Africa, Latin-America) in Mid-Atlantic Land Grant Research University (WVU) participated in the quantitative part of this research. The list of the contact information of international graduate students was obtained from the Office of International Students and Scholars (OISS). The survey was also delivered to international graduate students during the 
international activities, events and festivals organized by the Office of International Students and Scholars (OISS).

4 international graduate students ( 2 male and 2 female) from four different areas (Europe, Africa, Asia and Latin-America) participated in the qualitative part of this research.

\section{Instrumentation}

An instrument designed by the researcher was applied to collect data on international graduate students' perceived social support and perceived academic success. This instrument is self-reported and it was administered to international graduate students in Mid-Atlantic Land Grant Research University (WVU). This instrument is composed of three parts: Demographic Information, Perceived Social Support and Perceived Academic Success. The Perceived Social Support section includes 20 five-point Likert Scale questions that require respondents to indicate the degree to which each of the statements is true of them (1-Strongly disagree, 2- Disagree, 3Neutral, 4- Agree, and 5- Strongly agree). This section is designed to measure four separate dimensions of international graduate students' perceived social support including academic support, emotional support, financial support and spiritual support. Responses to these 20 questions indicated the degree to which that respondents have perceived the availability of social support. Categories with averages of 4.0 or over were considered as the most satisfying perceived social support of the respondents. Categories with averages of 3 to 4 were considered as normal perceived social support. Categories with averages lower than 3 were considered as problematic perceived social support.

The Perceived Academic Success section includes 6 four-point ordinal scale questions which describe six aspects of international graduate students' perceived academic success including knowledge learning, research skills, employability, potential earning, professional 
development and comparative ability. Respondents need to choose the option that best describes their situation of perceived academic success (1-Unsuccessful, 2-Somewhat successful, 3Successful, 4- Very successful). An average over 3.5 was considered as satisfying perceived academic success in this specific aspect.

The Perceived Social Support and the Perceived Academic Success sections of this instrument was preceded by a demographic section that is designed by the researcher to collect data concerning the participants' gender, age, region, academic year, and GPA information. These demographic data of the participants was essential for the analysis of this research.

This instrument (International Graduate Students Social Support Survey) has been designed for this study according to international graduate students' possible adjustment problems when they study abroad. It has been tested for internal reliability and has been identified to have high levels of reliability. The Perceived Social Support section of this instrument has demonstrated high reliability with academic support frame having a Cronbach alpha of 0.85 ; emotional support frame of 0.83 ; financial support frame of 0.86 and spiritual support frame of 0.83. The Perceived Academic Success section of this instrument has also demonstrated high reliability with knowledge learning item having a Cronbach alpha of 0.80; research skills item of 0.85 ; employability item of 0.82 ; potential earning item of 0.83 ; professional development item of 0.85 and comparative ability item of 0.80 . According to Field (2009), if the value of Cronbach alpha is around or higher than .8, there's no need to concern about the reliability of the survey items of an instrument. This instrument can be considered to be reliable to be applied in a research. The value of Cronbach alpha of each item in this instrument has been tested through SPSS (Statistical Package for Social Sciences), and they are all around or higher than 0.80 . Thus this instrument can be considered to be reliable without any item greatly 
affecting the overall reliability, and no item should cause a substantial decrease in the value of Cronbach alpha.

For the qualitative part, 20 interview questions were designed based on research question three. These questions were grouped into five categories including Academic support, Emotional support, Financial support, Spiritual support and GPA.

\section{Data Collection}

The researcher created a mailing list of the international graduate students from five regions in the world (South-East Asia, Middle-East, Europe, Africa and Latin-America) in MidAtlantic Land Grant Research University (WVU). The international graduate students' contact information was obtained from the Office of International Students and Scholars (OISS) in MidAtlantic Land Grant Research University (WVU). This list included the participants' name, email address and phone number. Through the application of survey monkey software, participants were sent an electronic version of the International Graduate Students Social Support Survey (IGSSSS). An email requesting acceptance to participate in this survey was sent and it demonstrated the purpose of this study and its objectives and also assured participants of data confidentiality (see appendix A for international graduate students' cover letter).

Responses from participants were expected to be received within two weeks, and those participants who haven't responded within this set time received automatic reminder from the researcher. Participants received follow-up emails twice in these two weeks after the initial email (see appendix B for international graduate students' reminder). Data collection for this research lasted four months after the initial date of this research. Participant confidentiality was ensured through the application of identity protection function of the survey monkey software and through the Internal Review Board (IRB) research corporation training. 
Interviews for the qualitative part of this research lasted two weeks. Transcribing work for the interviews lasted for another two weeks before the qualitative data analysis started.

\section{Data Analysis}

For research questions 1 and 2, the responses collected through the survey instrument were processed in an SPSS (Statistical Package for the Social Sciences) data file. The statistical method to analyze the correlation between international graduate students' demographic variables and perceived social support and academic success (GPA and perceived academic success) were determined by the following research questions:

1. Can we predict international graduate students' GPA from measures of gender, age, region, year, academic support, emotional support, financial support and spiritual support?

Descriptive statistics were applied to analyze this research question. Pearson Correlations were also applied to determine if there's a significant correlation between international graduate students' GPA and the predictors. A multiple regression model was conducted to determine if international graduate students' GPA can be predicted from measures of gender, age, region, year, academic support, emotional support, financial support and spiritual support.

2. Can we predict international graduate students' perceived academic success from measures of gender, age, region, year, academic, emotional, financial and spiritual supports?

Descriptive statistics were applied to analyze this research question. Pearson Correlations was applied to determine if there's a significant correlation between international graduate students' perceived social support and perceived academic success. A multiple regression model was also conducted to determine if international graduate students' perceived academic success 
can be predicted from measures of gender, age, region, year, academic support, emotional support, financial support and spiritual support.

Research question 3 was designed for the qualitative part of this study. In this section, a phenomenological method was applied to interpret international graduate students' perception of their academic success and social support system in Mid-Atlantic Land Grant Research University.

3. How do international graduate students perceive their academic success and social support system at Mid-Atlantic Land Grant Research University?

The phenomenological research method was conducted to analyze international graduate students' lived experiences of academic success and social support when they study in MidAtlantic Land Grant Research University. The researcher interviewed 4 individual international graduate students ( 2 males and 2 females) from four areas (Middle-East, Africa, Europe and Latin-America) to collect the descriptive data about international graduate students' study abroad experiences related with their academic success and social support.

Table 3.1

Research Questions and Models

\begin{tabular}{|l|l|}
\hline Research Questions & \\
\hline $\begin{array}{l}\text { Q1. Can we predict } \\
\text { international graduate }\end{array}$ & \\
students' GPA from measures & Model 1. GPA $=$ \\
of gender, age, region, year, & $\mathrm{b}_{0}+\mathrm{B}_{1}$ gender $+\mathrm{B}_{2}$ age $+\mathrm{B}_{3}$ region $+\mathrm{B}_{4}$ year $+\mathrm{B}_{5}$ academicsupport $+\mathrm{B}_{6}{\text { emotionalsupport }+\mathrm{B}_{7} \text { financialsupport }+\mathrm{B}_{8} \text { spiritualsupport }}^{\text {academic support, emotional }}$ \\
support, financial support and & \\
spiritual support? & \\
\hline
\end{tabular}




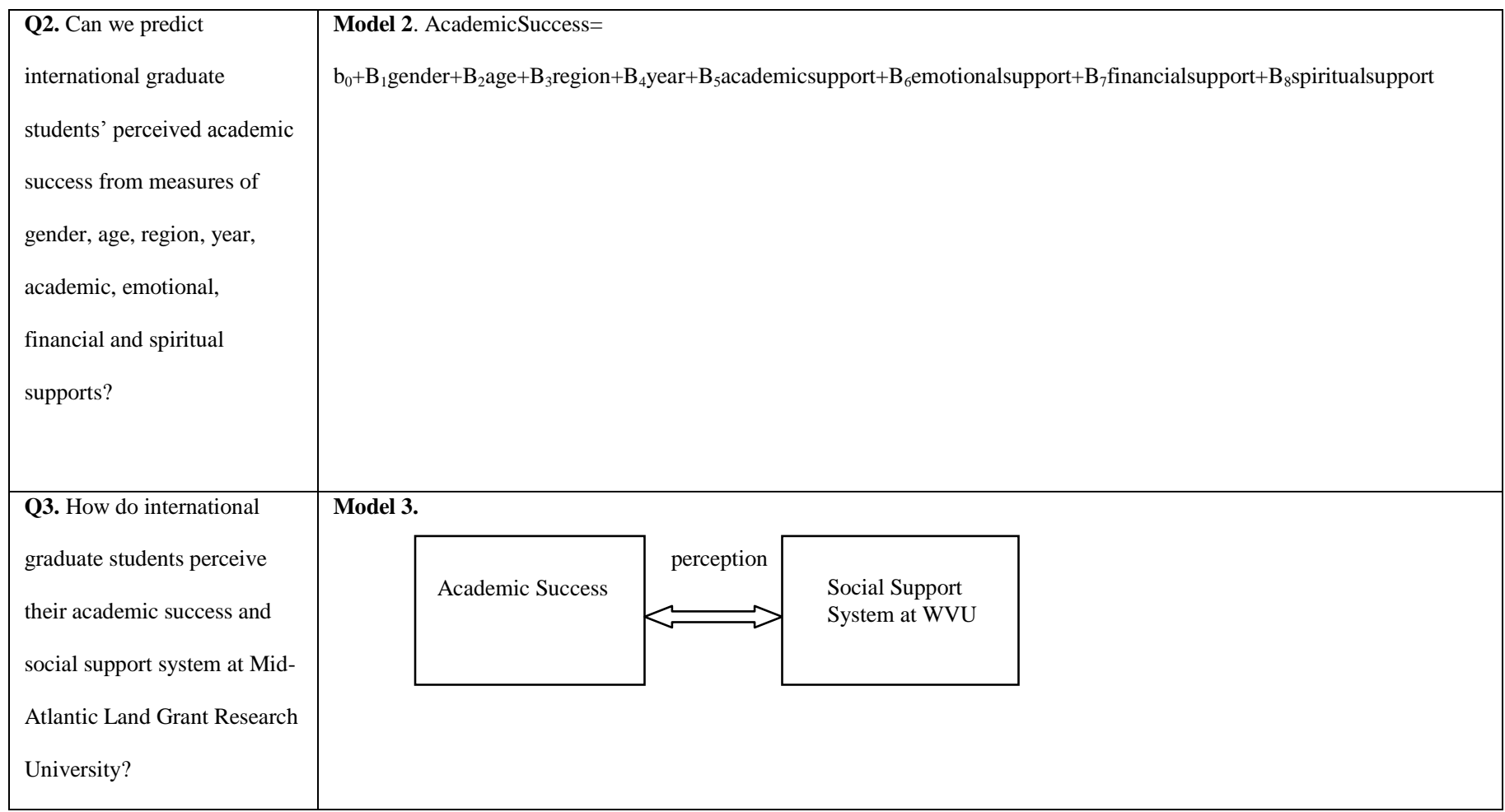

Note: 1. Model 1.and Model 2.are multiple regression models based on the general equation of multiple regression formula: $Y_{i}=b_{0}+b_{1} X_{1}+b_{2} X_{2}+\cdots+b_{n} X_{n}$

2. Predictors: $b_{0}$ (Constant); Gender; Age; Region; Year; Academic Support; Emotional Support; Financial Support; Spiritual Support.

3. Dependent Variables: GPA; Academic Success.

4. Unstandardized Regression Coefficients: Bs.

5. Model 3.is about international graduate students' perception of academic success and social support system at WVU. 


\section{Chapter IV}

\section{Findings}

\section{Introduction}

This study examined whether correlations existed between the dependent variables (GPA and perceived academic success) and the independent variables (gender, age, region, year, academic support, emotional support, financial support and spiritual support). International graduate students' understanding of academic success and social support system in Mid-Atlantic Land Grant Research University was also analyzed through the application of phenomenological method.

The population for the quantitative research part of this study included 100 international graduate students. They all completed and returned the survey. Thus all of the 100 cases were assessed as valid data and were all used in this study. The population for the qualitative research part of this study involved 4 international graduate students ( 2 males and 2 females). Interviews were conducted to collect descriptive data for this section.

For the quantitative part, two multiple regression models were conducted to present research question 1 and 2 . This part has been organized into 5 sections according to these two research questions. Section 1 interpreted the descriptive findings of the independent variables and dependent variables about their means and standard deviations. Section 2 applied the Pearson correlation analysis to examine the relationship between the independent variables (predictors) and dependent variables (outcomes). Section 3 conducted two multiple regression models according to research question 1 and 2 to examine the significance of the model, the significance and contribution of the predictor variables to the outcome variables. Section 4 
examined the validity of the multiple regression models. Finally section 5 assessed the generalization of the multiple regression models.

\section{Descriptive Findings}

Independent variables. As we can see in Table 4.1below, the sample size of this study was 100 international graduate students at Mid-Atlantic Land Grant Research University. The means of the independent variables were Gender (.53), Age (2.00), Region (2.83), Year (2.40), AcademicSupport (2.9087), EmotionalSupport (2.8700), FinancialSupport (3.5280), SpiritualSupport (2.8840). The standard deviations of the independent variables were Gender (.502), Age (.711), Region (1.349), Year (.899), AcademicSupport (.63183), EmotionalSupport (.65157), FinancialSupport(.34527), SpiritualSupport (.60914).

Table 4.1

Means and Standard Deviations of Independent Variables $(\mathrm{N}=100)$

\begin{tabular}{|l|l|l|l|}
\hline Independent Variables & Mean & Std. Deviation & N \\
\hline Gender & .53 & .502 & 100 \\
Age & 2.00 & .711 & 100 \\
Region & 2.83 & 1.349 & 100 \\
Year & 2.40 & .899 & 100 \\
AcademicSupport & 2.9087 & .63183 & 100 \\
EmotionalSupport & 2.8700 & .65157 & 100 \\
FinancialSupport & 3.5280 & .34527 & 100 \\
SpiritualSupport & 2.8840 & .60914 & 100 \\
\hline
\end{tabular}

Note: Results were produced based on untransformed data.

The descriptive findings of independent variables indicated that:

1. International graduate students participated in this research were about half males and half females. 
2. Most of the international graduate students participated in this research were at the age range of 25 to 35 .

3. $57 \%$ of the international graduate students participated in this research were from South, East Asian; Middle-East; European countries. Another 43\% were from African and Latin-American countries.

4. $60 \%$ of the international graduate students participated in this research have stayed in U.S. for 1 to 5 years.

5. $58 \%$ of the international graduate students participated in this research found it not easy to register for the courses through WVU STAR system; and there was no ample support for them to enhance their English language skills; and they have been able to find an international support group to talk about their exam anxiety; and they were not concerned about their writing ability in English; and the academic advising for international graduate students was not considered to be excellent.

6. $56 \%$ of the international graduate students participated in this research believed that WVU had considered the emotional support that international graduate students would need; and international graduate students would not feel inferior to others because of the cultural differences; and finding friends was not easy; and many international graduate students would not feel uneasy about the sexual customs in U.S.; and there was no transition program that helped them to build a good relationship with their roommate $(\mathrm{s})$.

7. $70 \%$ of the international graduate students participated in this research believed that the tuition waiver program for international graduate students at WVU was excellent; and there were ample graduate assistantships for international graduate students at 
WVU; and there was a shortage of part-time jobs on campus for international graduate students at WVU; and there were a good number of part-time jobs off campus for international graduate students at WVU; and many international graduate students didn't have enough money to meet their financial expenses.

8. $56 \%$ of the international graduate students participated in this research believed that accesses to multiple faiths of the world were not limited in and around WVU; and the multiple faiths of U.S. didn't make it confusing for international graduate students; and they didn't find it difficult to hear people criticize a certain faith in an open discussion; and they would know where to go if they need spiritual guidance.

Dependent variables. As we can see in Table 4.2 below, the means of the dependent variables were GPA (3.5650), AcademicSuccess (3.2601). The standard deviations of the dependent variables were GPA (.17137), AcademicSuccess (1.88572).

Table 4.2

Means and Standard Deviations of dependent Variables $(\mathrm{N}=100)$

\begin{tabular}{|l|l|l|l|}
\hline Dependent Variables & Mean & Std. Deviation & N \\
\hline GPA & 3.5650 & .17137 & 100 \\
AcademicSuccess & 3.2601 & 1.88572 & 100 \\
\hline
\end{tabular}

Note: Results were produced based on untransformed data.

The descriptive findings of dependent variables indicated that:

1. $90 \%$ of the international graduate students participated in this research had a GPA of 3.5 to 4.0 . 
2. $82 \%$ of the international graduate students participated in this research believed that the level of their knowledge learning through their program at WVU was successful; and their research skills were successful; and their program at WVU to improve their employability was successful; and their study related to their potential earning was successful; and their study at WVU related to their professional development was successful; and their study at WVU was successful compared to other international graduate students.

\section{Pearson Correlation Analysis}

The Pearson correlation analysis was conducted to examine the relationships between the independent variables or predictor variables (Gender, Age, Region, Year, AcademicSupport, EmotionalSupport, FinancialSuppport and EmotionalSupport) and the dependent variables or outcome variables (GPA and AcademicSuccess). Thus Pearson's correlation matrix (Table 4.3) and significant levels of correlation coefficients were reported at $a$-level of .50 .

As we can see in Table 4.3, the results of the Pearson correlation analysis indicated that:

1. The outcome variable GPA- this dependent or outcome variable was significantly related with six out of the eight independent or predictor variables, with correlation coefficients of .28 (GPA/Gender), .26 (GPA/Year), .48 (GPA/AcademicSupport), .26 (GPA/EmotionalSupport), .37 (GPA/FinancialSupport), .16 (GPA/SpiritualSupport).

Six Pearson correlation coefficients (Gender, Year, AcademicSupport, EmotionalSupport, FinancialSupport and SpiritualSupport) were positively significant at $a$-level of $<.05$; However, the predictor variables of Age and Region were not significantly correlated with the outcome variable GPA with correlation coefficients of .14 (GPA/Age), .16 (GPA/Region), at $a$-level >.05. 
2. The outcome variable AcademicSuccess- this dependent or outcome variable was significantly related with all the independent or predictor variables, with correlation coefficients of .36 (AcademicSuccess/Gender), .17 (AcademicSuccess/Age), .13 (AcademicSuccess/Region), .23 (AcademicSuccess/Year), .78 (AcademicSuccess/AcademicSupport), .38 (AcademicSucess/EmotionalSupport), .59 (AcademicSuccess/FinancialSupport), .22 (AcademicSuccess/SpiritualSupport). All the Pearson correlation coefficients were positively significant at $a$-level of <.05.

Table 4.3

Pearson Correlation Matrix $(N=100)$

\begin{tabular}{|c|c|c|c|c|c|c|c|c|c|}
\hline & & 1 & 2 & 3 & 4 & 5 & 6 & 7 & 8 \\
\hline 1 & Gender & & & & & & & & \\
\hline 2 & Age & $.20 *$ & & & & & & & \\
\hline 3 & Region & $.19 *$ & $.16^{*}$ & & & & & & \\
\hline 4 & Year & $.24 *$ & $.12 *$ & $.08 *$ & & & & & \\
\hline 5 & AcademicSupport & $.26 * *$ & $.20 *$ & $.10 *$ & $.16 * *$ & & & & \\
\hline 6 & EmotionalSupport & $.40 * *$ & $.22 *$ & $.15^{*}$ & $.14 * *$ & $.12 * *$ & & & \\
\hline 7 & FinancialSupport & $.27 * *$ & $.25 *$ & $.18^{*}$ & $.12 * *$ & $.15^{* *}$ & $.06^{*}$ & & \\
\hline 8 & SpiritualSupport & $.45^{* *}$ & $.39 * *$ & $.20 *$ & $.09 * *$ & $.06 *$ & $.05^{*}$ & $.07 * *$ & \\
\hline 9 & GPA & $.28 *$ & .14 & .16 & $.26^{*}$ & $.48 * * *$ & $.26 * *$ & $.37 * *$ & $.16^{* *}$ \\
\hline 10 & AcademicSuccess & $.36 *$ & $.17 * *$ & $.13 * *$ & $.23 * *$ & $.78 * * *$ & $.38 * * *$ & $.59 * * *$ & $.22 * *$ \\
\hline
\end{tabular}

Note: Dependent Variables: GPA and AcademicSuccess. Significant Levels: * $p<.05 ; * * p<.01$; $* * * p<.001$ (one-tailed). 
Thus, according to the Pearson correlation analysis results, we can reach the conclusion that six out of the eight predictor variables were significant (Gender, Year, AcademicSupport, EmotionalSupport, FinancialSupport, SpiritualSupport ) to predict the outcome variable GPA; Two predictor variables (Age, Region) may not be good predictors for the outcome variable GPA. All of the predictor variables (Gender, Age, Region, Year, AcademicSupport, EmotionalSupport, FinancialSupport, SpiritualSupport) were significant to predict the outcome variable AcademicSuccess. Hence, multiple regression models have been established to further examine the relationships between the predictor variables and outcome variables.

\section{Multiple Regression Models}

Multiple regression model 1. The first multiple regression model was established according to research question one which stated: Can we predict international graduate students' GPA from measures of gender, age, region, year, academic support, emotional support, financial support and spiritual support? Regression model 1 was expressed as: GPA= $\mathrm{b}_{0}+\mathrm{B}_{1}$ gender $+\mathrm{B}_{2}$ age $+\mathrm{B}_{3}$ region $+\mathrm{B}_{4}$ year $+\mathrm{B}_{5}$ academicsupport $+\mathrm{B}_{6}$ emotionalsupport $+\mathrm{B}_{7}$ financialsup port $+\mathrm{B}_{8}$ spiritualsupport

The independent variables in multiple regression model 1 were Gender, Age, Region, Year, AcademicSupport, EmotionalSupport, FinancialSupport and SpiritualSupport; and the dependent variable was GPA.

The equation of multiple regression model 1. The equation of multiple regression model 1 was developed according to the values of constant and unstandardized coefficients $(B \mathrm{~s})$ of the eight predictors. As we can see in Table 4.4 below, the constant was 0.988 ; and the unstandardized coefficients for the eight predictors were 0.24 (Gender), 0.18 (Age), 0.12 
(Region), 0.20 (Year), 0.88 (AcademicSupport), 0.35 (EmotionalSupport), 0.50

(FinancialSuppport), 0.16 (SpiritualSupport).

Table 4.4

Results of Standard Multiple Regression Analysis for the Regression Model $1(N=100)$

\begin{tabular}{|l|ccc|}
\hline Variables & $B$ & $S E B$ & $\beta$ \\
Constant & 0.988 & 0.17 & $.18^{*}$ \\
Age & 0.24 & 0.05 & .16 \\
Region & 0.18 & 0.07 & .14 \\
Year & 0.12 & 0.03 & .19 \\
AcademicSupport & 0.20 & 0.06 & $.45^{* *}$ \\
EmotionalSupport & 0.88 & 0.17 & $.26^{*}$ \\
FinancialSupport & 0.35 & 0.04 & $.30^{*}$ \\
SpiritualSupport & 0.50 & 0.08 & $.12^{*}$ \\
\hline
\end{tabular}

Note: Predictors: (Constant), Gender, Age, Region, Year, AcademicSupport, EmotionalSupport, FinancialSupport, SpiritualSupport. Dependent Variable: GPA. Significant Levels: * $p<.05$; ** $p<.01 ; * * * p<.001$.

As I discussed above, multiple regression model 1 of this study was expressed as: $\mathrm{GPA}=\mathrm{b}_{0}+\mathrm{B}_{1}$ gender $+\mathrm{B}_{2}$ age $+\mathrm{B}_{3}$ region $+\mathrm{B}_{4}$ year $+\mathrm{B}_{5}$ academicsupport $+\mathrm{B}_{6}$ emotionalsupport $+\mathrm{B}_{7}$ finan cialsupport $+\mathrm{B}_{8}$ spiritualsupport. Thus the formulation of this model was: GPA= 
$0.988+0.24$ gender+0.18age+0.12region+0.20year+0.88academicsupport+0.35emotionalsupport+ 0.50financialsupport+0.16spiritualsupport.

The significance of regression model 1. As we can see in Table 4.5 below, the results of the summary of multiple regression model 1 indicated that this model was statistically significant with $\mathrm{R}=.622, \mathrm{R}^{2}=.386$, adjusted $\mathrm{R}^{2}=.364, \mathrm{~F}(8,91)=22.089, p<.05$. The eight predictors together accounted for $36.4 \%$ of variance on the outcome variable GPA.

Table 4.5

Summary of Multiple Regression Model $1(N=100)$

\begin{tabular}{|l|l|l|l|l|l|l|l|l|}
\hline Model & R & R Square & $\begin{array}{l}\text { Adjusted } \\
\text { R Square }\end{array}$ & $\begin{array}{l}\text { R Square } \\
\text { Change }\end{array}$ & df1 & df2 & F & Durbin- \\
\hline 1 & .622 & .386 & .364 & .386 & 8 & 91 & $22.089^{*}$ & 1.876 \\
\hline
\end{tabular}

Note: Predictors: (Constant), Gender, Age, Region, Year, AcademicSupport, EmotionalSupport, FinancialSupport, SpiritualSupport. Dependent Variable: GPA. Significant Levels: * $p<.05$; ** $p<.01 ; * * * p<.001$

The significance of predictor variables. As we can see in Table 4.4, the values of unstandardized coefficients $B$, the standardized coefficients $\beta$, and t-tests with significant $a$-level of .05 were examined to evaluate the significance of the predictor variables.

The results of Table 4.4 told us that five out of eight predictor variables (Gender, AcademicSupport, EmotionalSupport, FinancialSupport, SpiritualSupport) had significant relationships with the outcome variable (GPA) with the values of standardized coefficients $\beta$ with $a$-level of <.05. However, three predictor variables (Age, Region, Year) had non-significant 
relationships with the outcome variable (GPA) with the values of standardized coefficients $\beta$ with $a$-level of $>.05$.

The regression correlations between the predictor variables and the outcome variable were further interpreted as below:

1. The predictor of Gender had a small size of regression correlation with the outcome variable GPA, with $\beta=.18, \mathrm{t}(100)=0.822$, and this correlation was positively significant with $a$-level of $<.05$.

2. The predictor of Age had non-significant regression correlation with the outcome variable GPA, with $\beta=.16, \mathrm{t}(100)=0.602$ with a-level of $>.05$.

3. The predictor of Region had non-significant regression correlation with the outcome variable GPA, with $\beta=.14, \mathrm{t}(100)=0.700$ with a-level of $>.05$.

4. The predictor of Year had non-significant regression correlation with the outcome variable GPA, with $\beta=.19, \mathrm{t}(100)=0.802$ with a-level of $>.05$.

5. The predictor of Academic Support had a big size of regression correlation with the outcome variable GPA, with $\beta=.45, \mathrm{t}(100)=4.889$, and this correlation was positively significant with $a$-level of <.01.

6. The predictor of Emotional Support had a small size of regression correlation with the outcome variable GPA, with $\beta=.26$, $\mathrm{t}(100)=3.609$, and this correlation was positively significant with $a$-level of $<.05$.

7. The predictor of Financial Support had a small size of regression correlation with the outcome variable GPA, with $\beta=.30, \mathrm{t}(100)=3.822$, and this correlation was positively significant with $a$-level of <.05. 
8. The predictor of Spiritual Support had a small size of regression correlation with the outcome variable GPA, with $\beta=.12, \mathrm{t}(100)=0.904$, and this correlation was positively significant with $a$-level of $<.05$.

As I have discussed above, the results of the Pearson correlation analysis (see Table 4.3) told us that there was no significant correlation between the predictor variable of Age and the outcome variable GPA with $r=.14, p>.05$; and there was also no significant correlation between the predictor variable of Region and the outcome variable GPA with $r=.16, p>.05$. Thus these two predictors were deleted from multiple regression model 1 . However, the results of the Pearson correlation analysis indicated that there was a significant correlation between the predictor variable of Year and the outcome variable GPA with $r=.26, p<.05$. Thus the post hoc evaluation was further conducted to see if the predictor variable of Year should be deleted from regression model 1.

As we can see in Table 4.6, it was found out that there was a significant regression correlation between the predictor variable of Year and the outcome variable GPA with $\beta=.28, \mathrm{t}$ $(100)=3.745$ at $a$-level of $<.05$ with this predictor variable served as the only independent variable in the regression model. This indicated that the regression correlation between the predictor variable of Year and the outcome variable GPA seemed to be mediated by the relationships between other predictor variables and the outcome variable GPA in regression model 1. Thus, the predictor variable of Year remained in multiple regression model 1. Table 4.6

\begin{tabular}{|l|c|c|c|c|}
\hline Variables & $B$ & $S E B$ & $\beta$ & $t$ \\
\hline Constant & 0.957 & 0.14 & & 16.552 \\
Year & 0.37 & 0.06 & $.28^{*}$ & 3.745 \\
\hline
\end{tabular}


Note: Predictors: (Constant), Year. Dependent Variable: GPA. Significant Levels: ${ }^{*} p<.05 ; * * p<$ $.01 ; * * * p<.001$

As a result, multiple regression model 1 was operated for the second time with six predictor variables including Gender, Year, AcademicSupport, EmotionalSupport, FinancialSupport and SpiritualSupport. The predictor variables of Age and Region were deleted from multiple regression model 1. As we can see in Table 4.7 below, the results of the summary of re-operated multiple regression model 1 indicated that this model was statistically significant with $\mathrm{R}=.624, \mathrm{R}^{2}=.389$, adjusted $\mathrm{R}^{2}=.375, \mathrm{~F}(6,93)=30.558, p<.01$. The six predictors together accounted for $37.5 \%$ of variance on the outcome variable GPA.

Table 4.7

Summary of Multiple Regression Model $1(N=100)$

\begin{tabular}{|l|l|l|l|l|l|l|l|l|}
\hline Model & R & R Square & Adjusted & R Square & df1 & df2 & F & Durbin- \\
R Square & Change & & & & & Watson \\
\hline 1 & .624 & .389 & .375 & .389 & 6 & 93 & $30.558^{* *}$ & 1.873 \\
\hline
\end{tabular}

Note: Predictors: (Constant), Gender, Year, AcademicSupport, EmotionalSupport, FinancialSupport, SpiritualSupport. Dependent Variable: GPA. Significant Levels: * $p<.05$; ** $p<.01 ; * * * p<.001$

The results of standard multiple regression analysis for the re-operated multiple regression model 2 (see Table 4.8) indicated that four of the predictor variables including AcademicSupport, EmotionalSupport, FinancialSupport and SpiritualSupport had significant regression correlation with the outcome variable GPA with $a$-level of <.05. However, two 
predictors variables including Gender and Year had non-significant regression correlation with the outcome variable GPA with $a$-level of $>.05$.

Table 4.8

Results of Standard Multiple Regression Analysis for the Regression Model $1(N=100)$

\begin{tabular}{|l|ccc|}
\hline Variables & $B$ & $S E B$ & $\beta$ \\
\hline Constant & 0.872 & 0.14 & .12 \\
Year & 0.20 & 0.04 & .15 \\
AcademicSupport & 0.18 & 0.03 & $.44^{* *}$ \\
EmotionalSupport & 0.86 & 0.14 & $.24^{*}$ \\
FinancialSupport & 0.28 & 0.03 & $.32^{* *}$ \\
SpiritualSupport & 0.54 & 0.12 & $.10^{*}$ \\
\hline
\end{tabular}

Note: Predictors: (Constant), Gender, Year, AcademicSupport, EmotionalSupport,

FinancialSupport, SpiritualSupport. Dependent Variable: GPA. Significant Levels: * $p<.05$; ** $p<.01 ; * * * p<.001$.

The results of Table 4.8 also told us that the constant was 0.872 , and unstandardized coefficients $(B)$ were 0.20 (Gender), 0.18 (Year), 0.86 (AcademicSupport), 0.28 (EmotionalSupport), 0.54 (FFinancialSupport), 0.12 (SpiritualSupport). Thus the formulation of the multiple regression model 1 was finally expressed as: GPA= 
$0.872+0.20$ gender+0.18year+0.86academicsupport+0.28emotionalsupport+0.54financialsupport +0.12 spiritualsupport.

The contribution of predictor variables. Pearson's correlation coefficients $R, R^{2}$, adjusted $R^{2}$ (see Table4.7), part correlation coefficients, and squared semi-partial correlation coefficients $\left(s r^{2}\right)$ (see Table 4.9) were examined in order to check the contribution of each predictor variable.

As we can see in Table 4.7, the value of adjusted $R^{2}=0.375$, and this indicated that the six predictors (Gender, Year, AcademicSupport, EmotionalSupport, FinancialSupport and SpiritualSupport) together accounted for $37.5 \%$ of variance on the outcome variable GPA.

The results of the summary of part and partial correlation analysis (see Table 4.9) told us that the part correlation coefficients for the six predictor variables were 0.067 (Gender), 0.062 (Year), 0.366 (AcademicSupport), 0.226 (EmotionalSupport), 0.305 (FinancialSupport), 0.102 (SpiritualSupport). And the squared semi-partial correlation coefficients for the four significant predictor variables were 0.085 (AcademicSupport), 0.035 (EmotionalSupport), 0.062 (FinancialSupport), 0.014 (SpiritualSupport). This indicated that (1) the significant predictor variable of AcademicSupport contributed to $8.5 \%$ of variance to the observed $\mathrm{R}^{2} ;(2)$ the significant predictor variable of EmotionalSupport contributed to $3.5 \%$ of variance to the observed $\mathrm{R}^{2}$; (3) the significant predictor variable of FinancialSupport contributed to $6.2 \%$ of variance to the observed $\mathrm{R}^{2}$; (4) the significant predictor variable of SpiritualSupport contributed to $1.4 \%$ of variance to the observed $\mathrm{R}^{2}$;

Additionally, we can also tell from Table 4.9 that the sum of significant $s r^{2}=.196$, and the observed $R^{2}=.389$, thus the difference between the observed $R^{2}$ and the significant $s r^{2}$ was 
.193. This indicated that the six predictor variables contributed $19.3 \%$ of variance to the observed $R^{2}$.

In summary, six out of eight predictor variable including Gender, Year, AcademicSupport, EmotionalSupport, FinancialSupport and SpiritualSupport had significant correlation with the outcome variable GPA through Pearson correlation analysis. After the reoperation of multiple regression model 1 without the predictor variables of Age and Region, four predictors including AcademicSupport, EmotionalSupport, FinancialSupport and SpiritualSupport had significant regression correlation with the outcome variable GPA.

Table 4.9

Summary of Part and Partial Correlation Coefficients $(N=100)$

\begin{tabular}{|lccc|}
\hline Variables & \multicolumn{3}{c|}{ Correlation } \\
\cline { 2 - 4 } & Partial & Part & $\mathrm{sr}^{2}$ \\
Gender & .092 & .067 & .062 \\
AcademicSupport & .084 & .366 & $.085^{* *}$ \\
EmotionalSupport & .506 & .226 & $.035^{*}$ \\
FinancialSupport & .320 & .305 & $.014 *$ \\
SpiritualSupport & .458 & .102 & \\
\hline
\end{tabular}

Note: Dependent Variable: GPA. Significant Levels: $* p<.05 ; * * p<.01 ; * * * p<.001$.

The accuracy of multiple regression model 1. In order to assess the accuracy of multiple regression model 1 , diagnostics of residuals and influential cases were examined; and 
also the analysis of standardized residuals, Cook's distance, centered leverage, Mahalanobis distances, standardized DFBeta, and the covariance ratio was selected on SPSS and examined.

Diagnostics of residuals. As we can see in table 4.10 below, three cases including case 29, case 40 and case 41 had standardized residuals greater than $2(2.018,-2.099,-2.016)$.

However, none of them had a value greater than 3.29. According to Andy Field (2009), if cases with standard residual values greater than 3.29, those cases should cause concern. Thus, we can believe that diagnostics of residuals in multiple regression model 1 were good enough not to cause any concern.

Table 4.10

Casewise Diagnostics for the Multiple Regression Model $1(N=100)$

\begin{tabular}{|l|r|r|r|r|}
\hline Case Number & Std. Residual & GPA & Predicted Value & Residual \\
\hline 29 & 2.018 & 3.90 & 3.0525 & .34746 \\
40 & -2.099 & 3.30 & 3.0613 & -.36133 \\
41 & -2.016 & 3.20 & 3.0471 & -.34708 \\
\hline
\end{tabular}

a. Dependent Variable: GPA

Diagnostics of influential cases. In order to evaluate the stability of multiple regression model 1, it was essential to check the inappropriate influence of any case in the regression model. Thus, Cook's distance, centered leverage, Mahalanobis distances, standardized DFBeta, and the covariance ratio were examined and reported as below:

1. Cook's distance. In multiple regression model 1, no values of Cook's distance of the cases were greater than 1 , and this indicated that there should be no concern about inappropriate overall influence of a case on the model.

2. Centered leverage. In multiple regression model 1, all of the leverage values were close to the value of $\mathrm{k}+1 / \mathrm{n}$, which was calculated as $6+1 / 100=0.07$ ( $\mathrm{k}$ represents the 
number of predictors which is 6 in this model, and $n$ represents the number of participants which is 100 in this model). This indicated that there was no inappropriate influence of cases over the model.

3. Mahalanobis distances. According to Barnett and Lewis (1978), a multiple regression model with a sample of 100, and predictors over 5, the value of Mahalanobis distances greater than 25 should cause concern. In this model, no values of Mahalanobis distances were beyond 25.

4. Standardized DFBeta. According to Stevens (2002), cases with absolute values of standardized DFBeta greater than 2 should cause concern. In this model, all absolute values of standard DFBeta were lower than 1.

5. Covariance ratio. According to Field (2009), the value of covariance ratio is close to 1 , the case is having very little influence on the variances of the model parameters. In this model, the values of covariance ratio were all close to 1 , ranging from 0.84 to 0.92 .

In summary, according to the analysis of the results of case wise diagnostics and diagnostics of influential cases, we can believe that multiple regression model 1 was fairly accurate and was stable across the sample.

The generalization of multiple regression model 1. To assess the generalization of multiple regression model 1, assumptions including variable types, Multicollinearity, Homoscedasticity, normality and linearity, independent errors and cross-validation were examined.

Assumptions of multiple regression model 1. To make conclusions about multiple regression model 1 , the following assumptions have been checked: 
1. Variable types. All predictor variables (Gender, Year, AcademicSupport, EmotionalSuppport, FinancialSupport, SpiritualSupport) were quantitative and categorical; and all outcome variables (GPA, AcademicSuccess) were quantitative and continuous. All predictor variables had some variation in value (no zero variance).

2. Multicollinearity. According to Field (2009), a correlation above 0.80 or 0.90 between two or more variables should cause concern for the multicollinearity of the regression model. As we can see in table 4.3, no Pearson correlation was higher than 0.80. Moreover, according to Myers (1990), a value of the variance inflation factor (VIF) higher than 10 and the average VIF greater than 1 should cause concern for the multicollinearity of the regression model. In multiple regression model 1 , the values of VIF were far lower than 10; and the VIF was lower than 1. Also, according to Menard (1995), the value of tolerance statistic below 0.2 should cause concern. The values of tolerance statistic were higher than 0.2 in this regression model.

3. Homoscedasticity, normality and linearity. As we can tell from the results of histogram of standard residuals and the normal P-P plot of regression standardized residuals (see appendix D), the homoscedasticity, normality and linearity of multiple regression model 1 were roughly met.

4. Independent errors. As we can see in Table 4.7, the value of Durbin-Watson was 1.873 which was close to 2 . This indicated that the residuals were independent from each other and there should be no concern for the independence of the residuals. Cross-Validation. Cross-validation of multiple regression model 1 was examined to see if this model can predict the outcome in a different sample. According to Field (2009), if a model 
can be generalized, it must be capable of accurately predicting the same outcome variable from the same set of predictors in a different group of people.

As we can see in Table 4.7, in this regression model, adjusted $R^{2}=0.375$. This means that the six predictors together accounted for $37.5 \%$ of variance in the outcome variable GPA. Additionally, the difference between the observed $R^{2}(0.389)$ and the adjusted $R^{2}(0.375)$ was 0.014, which was lower than 0.05 . This indicated that the cross-validation of multiple regression model 1 should not cause concern.

Sample size. According to Field (2009), there should be at least 10 cases of data for each predictor in the regression model. Green (1991) further suggested two rules for the minimum acceptable sample size for a multiple regression model. First, to check the overall significance of the multiple regression model, the recommended minimum sample size should be $50+8 \mathrm{k}$, where $\mathrm{k}$ represents the number of predictors; Second, to check the significance of individual predictors, the recommended minimum sample size should be $104+\mathrm{k}$.

In this multiple regression model, there were 6 predictors after deleting 2 predictors (Age and Region). Thus it met the requirements of the minimum sample size $(50+8 \times 6=98)$ to check the overall significance of this regression model. However, it didn't meet the requirements of the minimum sample size $(104+6=110)$ to check the significance of individual predictors.

In summary, the results of checking the accuracy and the generalization of multiple regression model 1 told us that this regression model was stable and could be roughly generalized and the predictor variables including Gender, Year, AcademicSupport, EmotionalSupport, FinancialSupport and Spiritual support could be applied to predict the outcome variable GPA . 
Multiple regression model 2. The second multiple regression model was established according to research question two which stated: Can we predict international graduate students' academic success from measures of gender, age, region, year, academic support, emotional support, financial support and spiritual support? Regression model 2 was expressed as: AcademicSuccess $=$ $\mathrm{b}_{0}+\mathrm{B}_{1}$ gender $+\mathrm{B}_{2}$ age $+\mathrm{B}_{3}$ region $+\mathrm{B}_{4}$ year $+\mathrm{B}_{5}$ academicsupport $+\mathrm{B}_{6}$ emotionalsupport $+\mathrm{B}_{7}$ financialsup port $+\mathrm{B}_{8}$ spiritualsupport

The independent variables in multiple regression model 2 were Gender, Age, Region, Year, AcademicSupport, EmotionalSupport, FinancialSupport and SpiritualSupport; and the dependent variable was AcademicSuccess.

The equation of multiple regression model 2. The equation of multiple regression model 2 was developed according to the values of constant and unstandardized coefficients $(B \mathrm{~s})$ of the eight predictors. As we can see in Table 4.11 below, the constant was 0.967 ; and the unstandardized coefficients for the eight predictors were 0.28 (Gender), 0.14 (Age), 0.17 (Region), 0.26 (Year), 0.89 (AcademicSupport), 0.36 (EmotionalSupport), 0.48 (FinancialSuppport), 0.26 (SpiritualSupport).

Table 4.11

Results of Standard Multiple Regression Analysis for the Regression Model $2(N=100)$

\begin{tabular}{|l|ccc|}
\hline Variables & $B$ & $S E B$ & $\beta$ \\
\hline Constant & 0.967 & 0.15 & .09 \\
Gender & 0.28 & 0.08 & .07 \\
Age & 0.14 & 0.05 & .10 \\
\hline
\end{tabular}




\begin{tabular}{|l|ccc|}
\hline Year & 0.26 & 0.06 & $.19 *$ \\
AcademicSupport & 0.89 & 0.18 & $.56^{* *}$ \\
EmotionalSupport & 0.36 & 0.09 & $.24 * *$ \\
FinancialSupport & 0.48 & 0.08 & $.28^{* *}$ \\
SpiritualSupport & 0.26 & 0.05 & $.10^{* *}$ \\
\hline
\end{tabular}

Note: Predictors: (Constant), Gender, Age, Region, Year, AcademicSupport, EmotionalSupport, FinancialSupport, SpiritualSupport. Dependent Variable: AcademicSuccess. Significant Levels: $* p<.05 ; * * p<.01 ; * * * p<.001$.

As I discussed above, multiple regression model 2 of this study was expressed as: AcademicSuccess $=\mathrm{b}_{0}+\mathrm{B}_{1}$ gender $+\mathrm{B}_{2}$ age $+\mathrm{B}_{3}$ region $+\mathrm{B}_{4}$ year $+\mathrm{B}_{5}$ academicsupport $+\mathrm{B}_{6}$ emotionalsup port $+\mathrm{B}_{7}$ financialsupport $+\mathrm{B}_{8}$ spiritualsupport. Thus the formulation of this model was:

AcademicSuccess $=$

$0.967+0.28$ gender+0.14age+0.17region +0.26 year+0.89academicsupport +0.36 emotionalsupport + 0.48financialsupport+0.26spiritualsupport.

The significance of regression model 2. As we can see in Table 4.12 below, the results of the summary of multiple regression model 2 indicated that this model was statistically significant with $\mathrm{R}=.735, \mathrm{R}^{2}=.540$, adjusted $\mathrm{R}^{2}=.536, \mathrm{~F}(8,91)=32.056, p<.01$. The eight predictors together accounted for $53.6 \%$ of variance on the outcome variable AcademicSuccess. Table 4.12 Summary of Multiple Regression Model $2(N=100)$

\begin{tabular}{|l|l|l|l|l|l|l|l|l|}
\hline Model & R & R Square & Adjusted & R Square & df1 & df2 & F & Durbin- \\
& & & R Square & Change & & & Watson \\
\hline
\end{tabular}




\begin{tabular}{|l|l|l|l|l|l|l|l|l|}
\hline 1 & .735 & .540 & .536 & .540 & 8 & 91 & $32.056^{* *}$ & 1.882 \\
\hline
\end{tabular}

Note: Predictors: (Constant), Gender, Age, Region, Year, AcademicSupport, EmotionalSupport, FinancialSupport, SpiritualSupport. Dependent Variable: AcademicSuccess. Significant Levels: $* p<.05 ; * * p<.01 ; * * * p<.001$.

The significance of predictor variables. As we can see in Table 4.11, the values of unstandardized coefficients $B$, the standardized coefficients $\beta$, and t-tests with significant $a$-level of .05 were examined to evaluate the significance of the predictor variables.

The results of Table 4.11 told us that five out of eight predictor variables (Year, AcademicSupport, EmotionalSupport, FinancialSupport, SpiritualSupport) had significant relationships with the outcome variable (AcademicSuccess) with the values of standardized coefficients $\beta$ with $a$-level of <.05. However, three predictor variables (Gender, Age, Region) had non-significant relationships with the outcome variable (AcademicSuccess) with the values of standardized coefficients $\beta$ with $a$-level of $>.05$.

The regression correlations between the predictor variables and the outcome variable were further interpreted as below:

1. The predictor of Gender had non-significant regression correlation with the outcome variable AcademicSuccess, with $\beta=.09, \mathrm{t}(100)=0.548$ with a-level of $>.05$.

2. The predictor of Age had non-significant regression correlation with the outcome variable AcademicSuccess, with $\beta=.07, \mathrm{t}(100)=0.608$ with a-level of $>.05$.

3. The predictor of Region had non-significant regression correlation with the outcome variable AcademicSuccess, with $\beta=.10, \mathrm{t}(100)=0.640$ with a-level of $>.05$. 
4. The predictor of Year had a small size of regression correlation with the outcome variable AcademicSuccess, with $\beta=.19, \mathrm{t}(100)=0.746$, and this correlation was positively significant with $a$-level of $<.05$.

5. The predictor of Academic Support had a big size of regression correlation with the outcome variable AcademicSuccess, with $\beta=.56, \mathrm{t}(100)=5.058$, and this correlation was positively significant with $a$-level of $<.01$.

6. The predictor of Emotional Support had a small size of regression correlation with the outcome variable AcademicSuccess, with $\beta=.24, \mathrm{t}(100)=3.580$, and this correlation was positively significant with $a$-level of $<.01$.

7. The predictor of Financial Support had a small size of regression correlation with the outcome variable AcademicSuccess, with $\beta=.28, \mathrm{t}(100)=3.663$, and this correlation was positively significant with $a$-level of $<.01$.

8. The predictor of Spiritual Support had a small size of regression correlation with the outcome variable AcademicSuccess, with $\beta=.10, \mathrm{t}(100)=0.899$, and this correlation was positively significant with $a$-level of $<.01$.

As I have discussed above, the results of the Pearson correlation analysis (see Table 4.3) told us that there were significant correlations between the predictor variables of Gender, Age, Region and the outcome variable AcademicSuccess with $r=.36, .17, .13$ respectively, $p<.05$. Thus the post hoc evaluation was further conducted to see if the predictor variables of Gender, Age, Region should be deleted from regression model 2.

As we can see in Table 4.13, 4.14, 4.15, it was found out that there was a significant regression correlation between the predictor variable of Gender and the outcome variable AcademicSuccess with $\beta=.14, \mathrm{t}(100)=4.028$ at $a$-level of $<.05$ with this predictor variable 
served as the only independent variable in the regression model. There was a significant regression correlation between the predictor variable of Age and the outcome variable AcademicSuccess with $\beta=.10, \mathrm{t}(100)=3.588$ at $a$-level of $<.05$ with this predictor variable served as the only independent variable in the regression model. There was a significant regression correlation between the predictor variable of Region and the outcome variable AcademicSuccess with $\beta=.19, \mathrm{t}(100)=4.922$ at $a$-level of $<.05$ with this predictor variable served as the only independent variable in the regression model. This indicated that the regression correlation between the predictor variables of Gender, Age, Region and the outcome variable AcademicSuccess seemed to be mediated by the relationships between other predictor variables and the outcome variable AcademicSuccess in regression model 2. Thus, the predictor variables of Gender, Age, Region remained in multiple regression model 2.

Table 4.13

\begin{tabular}{|l|c|c|c|c|}
\hline Variables & $B$ & SE B & $\beta$ & $t$ \\
\hline Constant & 0.948 & 0.12 & & 15.447 \\
Gender & 0.34 & 0.04 & $.14^{*}$ & 4.028 \\
\hline
\end{tabular}

Note: Predictors: (Constant), Gender. Dependent Variable: AcademicSuccess. Significant Levels: $* p<.05 ; * * p<.01 ; * * * p<.001$.

Table 4.14

\begin{tabular}{|l|c|c|c|c|}
\hline Variables & $B$ & $S E B$ & $\beta$ & $t$ \\
\hline Constant & 0.933 & 0.10 & & 14.049 \\
Age & 0.28 & 0.03 & $.10^{*}$ & 3.588 \\
\hline
\end{tabular}


Note: Predictors: (Constant), Age. Dependent Variable: AcademicSuccess. Significant Levels: * $p<.05 ; * * p<.01 ; * * * p<.001$.

Table 4.15

\begin{tabular}{|l|c|c|c|c|}
\hline Variables & $B$ & $S E B$ & $\beta$ & $t$ \\
\hline Constant & 0.992 & 0.17 & & 17.024 \\
Region & 0.39 & 0.08 & $.19 *$ & 4.922 \\
\hline
\end{tabular}

Note: Predictors: (Constant), Region. Dependent Variable: AcademicSuccess. Significant Levels: $* p<.05 ; * * p<.01 ; * * * p<.001$.

The contribution of predictor variables. Pearson's correlation coefficients $R, R^{2}$, adjusted $R^{2}$ (see Table 4.12), part correlation coefficients, and squared semi-partial correlation coefficients $\left(s r^{2}\right)$ (see Table 4.16) were examined in order to check the contribution of each predictor variable.

As we can see in Table 4.12, the value of adjusted $R^{2}=0.536$, and this indicated that the eight predictors (Gender, Age, Region, Year, AcademicSupport, EmotionalSupport, FinancialSupport and SpiritualSupport) together accounted for 53.6\% of variance on the outcome variable AcademicSuccess.

The results of the summary of part and partial correlation analysis (see Table 4.16) told us that the part correlation coefficients for the eight predictor variables were 0.059 (Gender), 
0.060 (Age), 0.063 (Region), 0.092 (Year), 0.376 (AcademicSupport), 0.208

(EmotionalSupport), 0.313 (FinancialSupport), 0.120 (SpiritualSupport). And the squared semipartial correlation coefficients for the five significant predictor variables were 0.012 (Year), 0.122 (AcademicSupport), 0.068 (EmotionalSupport), 0.104 (FinancialSupport), 0.096 (SpiritualSupport). This indicated that (1)the significant predictor variable of Year contributed to $1.2 \%$ of variance to the observed $\mathrm{R}^{2}$; (2) the significant predictor variable of AcademicSupport contributed to $12.2 \%$ of variance to the observed $\mathrm{R}^{2}$; (3) the significant predictor variable of EmotionalSupport contributed to $6.8 \%$ of variance to the observed $\mathrm{R}^{2}$; (4) the significant predictor variable of FinancialSupport contributed to $10.4 \%$ of variance to the observed $\mathrm{R}^{2} ;(5)$ the significant predictor variable of SpiritualSupport contributed to $9.6 \%$ of variance to the observed $\mathrm{R}^{2}$;

Additionally, we can also tell from Table 4.16 that the sum of significant $s r^{2}=.402$, and the observed $R^{2}=.540$, thus the difference between the observed $R^{2}$ and the significant $s r^{2}$ was .138. This indicated that the eight predictor variables contributed $13.8 \%$ of variance to the observed $R^{2}$.

In summary, the eight predictor variable including Gender, Age, Region, Year, AcademicSupport, EmotionalSupport, FinancialSupport and SpiritualSupport all had significant correlation with the outcome variable AcademicSuccess through Pearson correlation analysis. After the operation of multiple regression model 2, five predictors including Year, AcademicSupport, EmotionalSupport, FinancialSupport and SpiritualSupport had significant regression correlation with the outcome variable AcademicSuccess. 
Table 4.16

Summary of Part and Partial Correlation Coefficients $(N=100)$

\begin{tabular}{|c|c|c|c|}
\hline \multirow[t]{2}{*}{ Variables } & \multicolumn{3}{|c|}{ Correlation } \\
\hline & Partial & Part & $\mathrm{sr}^{2}$ \\
\hline Gender & .088 & .059 & \\
\hline Age & .090 & .060 & \\
\hline Region & .095 & .063 & \\
\hline Year & .150 & .092 & $.012 *$ \\
\hline AcademicSupport & .528 & .376 & $.122 * *$ \\
\hline EmotionalSupport & .306 & .208 & $.068 * *$ \\
\hline FinancialSupport & .469 & .313 & $.104 * *$ \\
\hline SpiritualSupport & .180 & .120 & $.096 *$ \\
\hline
\end{tabular}

Note: Dependent Variable: AcademicSuccess. Significant Levels: $* p<.05 ; * * p<.01 ; * * * p<$ .001.

The accuracy of multiple regression model 2. In order to assess the accuracy of multiple regression model 2, diagnostics of residuals and influential cases were examined; and 
also the analysis of standardized residuals, Cook's distance, centered leverage, Mahalanobis distances, standardized DFBeta, and the covariance ratio was selected on SPSS and examined.

Diagnostics of residuals. As we can see in table 4.17 below, two cases including case 1 and case 43 had standardized residuals greater than $2(-2.173,2.153)$. However, none of them had a value greater than 3.29. According to Andy Field (2009), if cases with standard residual values greater than 3.29 , those cases should cause concern. Thus, we can believe that diagnostics of residuals in multiple regression model 2 were good enough not to cause any concern. Table 4.17

\section{Casewise Diagnostics ${ }^{\mathrm{a}}$}

\begin{tabular}{|l|r|r|r|r|}
\hline Case Number & Std. Residual & AcademicSuccess & Predicted Value & Residual \\
\hline 1 & -2.173 & 1.43 & 5.0376 & -3.60804 \\
43 & 2.153 & 7.69 & 2.7970 & 4.89602 \\
\hline
\end{tabular}

a. Dependent Variable: AcademicSuccess

Diagnostics of influential cases. In order to evaluate the stability of multiple regression model 2, it was essential to check the inappropriate influence of any case in the regression model. Thus, Cook's distance, centered leverage, Mahalanobis distances, standardized DFBeta, and the covariance ratio were examined and reported as below:

1. Cook's distance. In multiple regression model 2, no values of Cook's distance of the cases were greater than 1 , and this indicated that there should be no concern about inappropriate overall influence of a case on the model.

2. Centered leverage. In multiple regression model 2, all of the leverage values were close to the value of $\mathrm{k}+1 / \mathrm{n}$, which was calculated as $8+1 / 100=0.09$ ( $\mathrm{k}$ represents the number of predictors which is 8 in this model, and $n$ represents the number of participants which is 100 in this model). This indicated that there was no inappropriate influence of cases over the model. 
3. Mahalanobis distances. According to Barnett and Lewis (1978), a multiple regression model with a sample of 100, and predictors over 5, the value of Mahalanobis distances greater than 25 should cause concern. In this model, no values of Mahalanobis distances were beyond 25.

4. Standardized DFBeta. According to Stevens (2002), cases with absolute values of standardized DFBeta greater than 2 should cause concern. In this model, all absolute values of standard DFBeta were lower than 1.

5. Covariance ratio. According to Field (2009), the value of covariance ratio is close to 1 , the case is having very little influence on the variances of the model parameters. In this model, the values of covariance ratio were all close to 1 , ranging from 0.88 to 0.95 .

In summary, according to the analysis of the results of case wise diagnostics and diagnostics of influential cases, we can believe that multiple regression model 2 was fairly accurate and was stable across the sample.

The generalization of multiple regression model 2. To assess the generalization of multiple regression model 2, assumptions including variable types, Multicollinearity, Homoscedasticity, normality and linearity, independent errors and cross-validation were examined.

Assumptions of multiple regression model 2. To make conclusions about multiple regression model 2 , the following assumptions have been checked:

1. Variable types. All predictor variables (Gender, Age, Region, Year, AcademicSupport, EmotionalSuppport, FinancialSupport, SpiritualSupport) were quantitative and categorical; and all outcome variables (GPA, AcademicSuccess) were quantitative 
and continuous. All predictor variables had some variation in value (no zero variance).

2. Multicollinearity. According to Field (2009), a correlation above 0.80 or 0.90 between two or more variables should cause concern for the multicollinearity of the regression model. As we can see in table 4.3, no Pearson correlation was higher than 0.80. Moreover, according to Myers (1990), a value of the variance inflation factor (VIF) higher than 10 and the average VIF greater than 1 should cause concern for the multicollinearity of the regression model. In multiple regression model 2 , the values of VIF were far lower than 10; and the VIF was lower than 1. Also, according to Menard (1995), the value of tolerance statistic below 0.2 should cause concern. The values of tolerance statistic were higher than 0.2 in this regression model.

3. Homoscedasticity, normality and linearity. As we can tell from the results of histogram of standard residuals and the normal P-P plot of regression standardized residuals (see appendix D), the homoscedasticity, normality and linearity of multiple regression model 2 were roughly met.

4. Independent errors. As we can see in Table 4.12, the value of Durbin-Watson was 1.882 which was close to 2 . This indicated that the residuals were independent from each other and there should be no concern for the independence of the residuals.

Cross-Validation. Cross-validation of multiple regression model 2 was examined to see if this model can predict the outcome in a different sample. According to Field (2009), if a model can be generalized, it must be capable of accurately predicting the same outcome variable from the same set of predictors in a different group of people. 
As we can see in Table 4.12, in this regression model, adjusted $\mathrm{R}^{2}=0.536$. This means that the eight predictors together accounted for $53.6 \%$ of variance in the outcome variable AcademicSuccess. Additionally, the difference between the observed $\mathrm{R}^{2}(0.540)$ and the adjusted $\mathrm{R}^{2}(0.536)$ was 0.004 , which was lower than 0.05 . This indicated that the cross-validation of multiple regression model 2 should not cause any concern.

Sample size. According to Field (2009), there should be at least 10 cases of data for each predictor in the regression model. Green (1991) further suggested two rules for the minimum acceptable sample size for a multiple regression model. First, to check the overall significance of the multiple regression model, the recommended minimum sample size should be $50+8 \mathrm{k}$, where $\mathrm{k}$ represents the number of predictors; Second, to check the significance of individual predictors, the recommended minimum sample size should be $104+\mathrm{k}$.

In this multiple regression model, there were 8 predictors. Thus it didn't meet the requirements of the minimum sample size $(50+8 \times 8=114)$ to check the overall significance of this regression model. And also it didn't meet the requirements of the minimum sample size $(104+8=112)$ to check the significance of individual predictors.

In summary, the results of checking the accuracy and the generalization of multiple regression model 2 told us that this regression model was stable and could be roughly generalized and the predictor variables including Gender, Age, Region, Year, AcademicSupport, EmotionalSupport, FinancialSupport and SpiritualSupport could be applied to predict the outcome variable AcademicSuccess.

\section{Research Question Three}

Research question three stated: How do international graduate students perceive their academic success and social support system at Mid-Atlantic Land Grant Research University? 
The interview questions were developed according to this research question. These interview questions were put into five categories involving international graduate students' perception of academic support, emotional support, financial support, spiritual support and GPA during their graduate study at WVU. However, these interview questions were just used to guide the conversation if it didn't go smoothly or it seemed that the interviewee tried to divert from the topic.

The transcripts were analyzed and five themes emerged based on the five categories of the interview questions. Since there were only four interviewees in this study including two male international graduate students and two female international graduate students from the areas of Europe, Africa, Asia and Latin-America, it was not difficult for the researcher to locate the dominant ideas of the interviewees about their perception of the issues belonging to the five themes.

Specifically, the descriptive phenomenological research method was conducted in this section to answer research question three which stated: How do international graduate students perceive their academic success and social support system at Mid-Atlantic Land Grant Research University? According to Welman and Kruger (1999), the aim of phenomenological research is to describe as accurately as possible the phenomenon, staying away from any presupposition, but remaining truth to the facts. They argued that "the phenomenologists are concerned with understanding social and psychological phenomena from the perspectives of people involved" (p.189). In this study, the researcher herself is an international graduate student at WVU, thus it has been difficult for her to keep herself away from the unique world of the participants. 
According to Moustakas (1994, p.103), there are six basic procedures to conduct phenomenological research method:

1. Discovering a topic and question rooted in autobiographical meanings and values, as well as involving social meanings and significance.

2. Constructing a set of criteria to locate appropriate participants.

3. Providing participants with instructions on the nature and purpose of the investigation, and developing an agreement that includes obtaining informed consent, ensuring confidentiality and delineating the responsibilities of the primary researcher and research participants, consistent with ethical principles of research.

4. Developing a set of questions or topics to guide the interview process.

5. Conducting and reporting a lengthy person-to-person interview that focuses on a bracketed topic and question.

6. Organizing and analyzing the data to facilitate development of individual textural and structural descriptions, a composite textural description, a composite structural description, and a synthesis of textural and structural meanings and essences. The six basic procedures of phenomenological research were conducted in this study as follows:

Topic selection. The researcher herself is an international graduate student. She has experienced various struggles belonging to the four categories of academic support, emotional support, financial support and spiritual support. Thus she was wondering that how other international graduate students perceive and deal with those difficulties. Within about three years, the title of The Social Support for International Graduate Students to Obtain Academic Success was finally developed and decided by the researcher. 
The researcher believed that this topic has social meaning and significance in the sense of empathizing with international graduate students for the purpose of improving their adaptation ability in a new learning environment and helping them obtain academic success.

Locating research participants. The researcher decided to choose the sampling purposively, considering the participants' gender, diversity in region, personality and so forth. Thus four participants were selected for this study. Two of them were male international graduate students, and the other two were female international graduate students. They were from four different regions including Europe, Africa, Asia and Latin-America. Also they were from different departments or colleges at WVU.

The researcher knew all of the participants before the interviews at different places. For example, she met some participants in class, and others at friends' place and so forth.

Protecting confidentiality. In order to protect the participants' confidentiality, the researcher developed a form of agreement with the participants based on Bailey's (1996) recommended items, including:

- That they are participating in research

- The purpose of the research

- The procedures of the research

- The risk and benefits of the research

- The voluntary nature of research participation

- The subjects' right to stop the research at any time

- The procedures used to protect confidentiality

Interview questions. The interview questions were put into five categories involving international graduate students' perception of academic support, emotional support, financial 
support, spiritual support and GPA during their graduate study at WVU. The following was the list of interview questions for this study:

\section{$\underline{\text { Academic support }}$}

1. Have you experienced any academic stress during your graduate study?

2. What is your perception of academic stress?

3. What types of academic issues have you encountered during your graduate study?

4. Have you received any support when you encountered these issues?

5. If yes, do you think these supports significantly affect the achievement of your academic success?

\section{Emotional support}

6. Have you experienced any emotional problems during your graduate study?

7. What types of emotional problems have you encountered?

8. Have you received any support when you encountered these problems?

9. If yes, do you think these supports importantly influence the achievement of your academic success?

\section{$\underline{\text { Financial support }}$}

10. Have you experienced any financial problems during your graduate study?

11. What types of financial problems have you encountered?

12. Have you received nay support when you encountered these problems?

13. If yes, do you think these supports significantly affect the achievement of your academic success?

\section{$\underline{\text { Spiritual support }}$}

14. Have you experienced any religious dilemma during your graduate study? 
15. What types of religious problems have you encountered?

16. Have you received any support when you encountered these problems?

17. If yes, do you think these supports importantly influence the achievement of your academic success?

\section{$\underline{\text { GPA }}$}

18. Do you think GPA is important aspect of academic success for your graduate study?

19. Do you think that social support you receive will significantly affect your GPA?

20. If yes, how social support will affect your GPA?

Conducting and reporting interviews. The researcher conducted unstructured in-depth interviews with the four participants. During this process, "bracketing" occurred for the purpose of maintaining the "pure subjectivity" of the participants in this study. The researcher focused on what's going on inside the participants and asked the participants to "describe the lived experience in a language free from the constructs of the intellect and the society as possible" (Bentz \& Shapiro, 1998. P. 96). At the same time, the researcher "bracketed her own preconceptions and entered into the participants' life world and used the self as an experiencing interpreter" (Miller \& Crabtree, 1992. P. 24).

The four interviews (totally 2 hours) took place individually in two weeks at quiet and safe places including one of the participant's office and others in the library. Unfortunately, one interview was interrupted by someone who stepped into the room. However, the interview went on and was finished eventually.

The researcher transcribed and reported all the interview data honestly and also stored them electronically on computer and flash drives in case of losing the data accidentally. 
Organizing and Analyzing data. The researcher organized and analyzed the interview data in five steps based on Hycner's (1999) phenomenological research process including:

1. Bracketing and phenomenological reduction.

2. Delineating units of meaning.

3. Clustering of units of meaning to form themes.

4. Summarizing each interview, validating it and where necessary modifying it.

5. Extracting general and unique themes from all the interviews and making a composite summary.

Bracketing and phenomenological reduction. The researcher "bracketed out" her own preconceptions or theoretical concepts and tried to enter the participants' unique world. The researcher listened to the recording of the interviews repeatedly in order to become familiar with the participants' interpretation of their lived experiences about studying abroad and maintain their own unique experiences.

Delineating units of meaning. While avoiding making inappropriate subjective judgements, the researcher carefully extracted the list of units of relevant meaning from each interview and deleted the redundant units.

Clustering of units of meaning to form themes. The researcher bracketed her own personal preconceptions again. She carefully examined the list of units of meaning and grouped them together. The researcher identified significant topics including five groups in this study and determined the central themes including Academic support and academic success, Emotional support and academic success, Financial support and academic success, Spiritual support and academic success, Social support and GPA. 
Summarizing each interview, validate and modify. The researcher went back to the participants and made sure that the essence of the interviews has been correctly summarized. General and unique themes for all the interviews and composite summary. The researcher generalized five common themes (Academic support and academic success, Emotional support and academic success, Financial support and academic success, Spiritual support and academic success, Social support and GPA) for all the interviews and wrote composite summaries.

\section{Theme one: Academic support and academic success.}

All the four participants expressed that they've experienced academic stress during their graduate study and necessary academic support would help alleviate those stresses. All the four participants believed that academic support would significantly affect the achievement of their academic success:

Participant 1: My perception of academic stress is from my standpoint would be the biggest challenge I had was the writing part because it is not my native language. The writing is done a little bit different then in Germany. You really have to shift the sentence structure, the way you set up the paper and then all the classroom discussions would be the academic stress too because in the beginning it is very hard to follow conversations but since many classrooms expect participation, you have a hard time participating if you are still trying to decipher what was talked about. It takes a while in the beginning to get quicker with the thinking and the speaking and the writing. That was for me personally my academic stress.

Participant 2: As a grad student you get a lot of work. For me, especially last year, I was during the course work for my Ph.D. and also writing my Master's thesis while being a 
teaching assistant and working on two papers in addition. It was quite stressful and a large workload.

Participant 3: I'm an international student so the system of educations is different in the requirements of students, the workload, the organization of work, and the expectations of professors. These things are different than what I am use to when I started working here. I have to combine teaching assistantship with graduate work, so I had more, not so much stress, but it takes more time. This time could be devoted to studying but instead goes towards grading and preparing for classes.

Participant 4: There was a time where I had some personal family problems and that kind of distracted me. Not because I didn't understand the material. I was just distracted that's the only time I had some stress academically.

The four participants have experienced different types of academic stress. Their descriptions about their academic stress show that international graduate students at WVU are working hard to deal with various academic difficulties:

Participant 1: In Germany it is more the lecturing types so basically you just sit there and you listen. It may have changed because I have been an international student for a while now but back when I got my undergraduate degree in Germany it was more of a lecturing style and really didn't ask questions. You took notes, you went home. You memorized them and you turned in a paper on the assignment but you didn't have a classroom discussion. It was an adjustment for me on a new learning style. It can be because if you don't understand what the discussion is about but $20-30 \%$ of your grade depends on participation, it puts pressure on you. You want to get a good grade but you don't k now how to get there. I don't understand even though you have 
good English skills but when you come here they speak so much faster and there is stuff you don't know translated and conversational English is a little different then academic English. When I came I was very good at speaking hello and how are you, talk about the weather and stuff but when you sit in a classroom and all of a sudden the academic words, it is like what do those mean? You have to learn second English I always called it. It was conversational English and then the academic English.

Participant 2: A lot of work that needed to be done. Computer science is quite a competitive field. The research is the most stressful part because anything involves a lot of work. Lots of reading, papers, and doing the research.

Participant 3: It's just my first semester in the doctorate program, and I haven't noticed any difference than with my master's program. Probably the content is different, it's broader and more vague than in my original field, I was doing Linguistics. What I like about it is that there are people with different backgrounds that I get to meet. I like that we can share experiences, that's what I really like about this doctorate program. I haven't been in the program for a long time but that is my first impression of it.

That most difficult part is that I have to start thinking about my dissertation but at the present moment I have some ideas and when I was writing my proposal I had some ideas that I thought I could develop but taking in taking the courses that I am no, they idea didn't lead me somewhere. I'm acquiring new information and I'm trying to adjust my interests to change them. My interests change every time I read an article, it's not that I change my ideas every day. I shape them differently; I try to approach my interests in different ways. It's the first semester but I know that three years will go by very fast; I have to start thinking about what I really want to do and not just waste time. 
I think you're right, I think the hardest part is to develop your interests but limit them to a specific topic. I think I need to find a professor to my interests and work with that professor to gain some experience on writing and researching. This apprenticeship is really important for masters and graduate students because we cannot just take our knowledge and experience from books and articles. We need hands on exercises like working with our particular person and seeing how that person works will probably lead you somewhere.

Participant 4: My brother passed away. My Mother was ill and struggling with it. Then his wife had their daughter after he had passed away. He was my only sibling and I had to step in to help. At that time I had a full load of courses. At the time I was struggling. I was kind of questioning whether there was point in trying to achieve all this academic stuff if you're not going to be around to benefit from it. It did affect my desire and motivation whether to continue my education, and question what the point was. That is how it affected me.

It was hard. I had to take some time off too. Even when I came back, I thought I was ready - I wouldn't say that I wasn't ready - I think my family wasn't ready because other things kept coming up. There was sadness and pain but that is part of trying to move on and the fallout of him passing away. I had to step in and help raise the daughter and provide support for my mother. It's one thing trying to deal with my emotional support, but I also had to provide emotional support for other people too. That took its toll. However, all the four participants believed that certain academic support would help relieve their academic stress, although they described the support they received differently:

Participant 1: When it comes to the speaking part, I received some support from the 
school in terms I was talking to friends and meeting a lot of people with good English and then you just practice, practice, practice just speaking. Like a friend of mine, she would read with me the local newspaper, The Dominion Post, and I would have to tell her if I didn't know certain expressions. She would explain it to me. That was very helpful. I do like to read so I think that helps a lot that I didn't mind doing all the reading like with the papers because that is how you pick up some of the words too and that helped me with the speaking part because I was getting better and better at the academic English. Within the school when it comes to speaking now because I think the school was not that aware. I can't remember that I was consciously approached and said look. Here is something that might help you. Not from the International Students Scholar office, not from the academic department and not from the University in general to my recollection and we are talking at the beginning of my program. I do know for a fact that things have changed now because they are more aware of that issue. You see more outlets addressing cultures, speaking and writing. That would be the other thing coming to the writing. There is no kind of support ever.

Not that I was aware of. So any department communication like an email to students, no and I don't think we had the Office of Communication then either so they have been working hard at addressing the writing issues now a days but I don't remember any before because I am only passed that point. The writing was also what I thought was not so good with a faculty member. When you turn in your papers, you do your best. But what I was missing was getting the feedback from the faculty. They would take the paper and you either had an A, B but you never got the paper back with either an A or B where it says you need to work on your grammar or you don't want to use this. Just some kind 
of feedback would have been really helpful. But if you did approach professors, it always sounded like they didn't have the time to do so. You either didn't find and don't worry about it but for the writing, no. I missed a lot. One of the faculty members was really good in leadership. He used a lot of red ink actually but there was only two and one of them left and the other one I only had two classes with those. It wasn't like over the course of your doctoral program you could some in the first semester and get the whole feedback but in the writing style, I never got that.

Participant 2: Yes I talked to a counselor from whatever WVU office it is and exercise usually helps also.

Participant 3: I receive great support from my colleagues. We work in the language department so we share the office with nineteen other TA's. Of course there are other people I can ask for help, in material support and spiritual support. It is important in the process and the coordinator of the Russian program I work for helps me a lot. She helps me to solve some problems if I encounter them, like managing the classroom. She gives me academic support sometimes, like how the American education system works. I can go talk to her and she can explain things to me. Probably the international office, I should mention it because I think it is doing a great job with integrating international students. With my first year in my master's program it helped me a lot. I am not that active with it because I do not have time, but I constantly receive emails about different trips, gatherings, and dinners. So I think it is a great support for international students to feel at home because a lot of them do not have their families here. They are doing a great job. Participant 4: Not really, because I didn't talk to people a lot, I dealt with it myself. Even my roommates didn't know. Culturally we don't talk a lot. We hide a lot of stuff. I 
didn't get any support but not because of the lack of it.

The participants believed that academic support would significantly affect the achievement of their academic success:

Participant 1: I can see it for people who really need it. I was just able then to work with what I had and then I had an internal drive to see if I could do better even though I don't have the help but I think if I would have had it would it have helped. It would have helped with the easing of the stress not necessarily I would have done better because I have grades that are ok but I think it would have helped especially when you get to the stage where you are writing your doctoral dissertation, all of a sudden you realize that it would have been really helpful if you would have had continued writing feedback. Because now you are writing 250 pages and you are like oh my, this is hard. No I am thinking he would have told me about the grammar. I wish they would have said you still think German but you need to write English. No I think it would have affected my academic success but the lack of support did not affect the academic success but I think it would help relieve some of the academic stress.

Participant 2: The support, yes I think it is beneficial. It helps you to deal with stress and how to deal with the increased workload.

Participant 3: I think so. There is no family around me that I can ask for help. Of course international office, my colleagues, my coordinator, or whoever any person needs this support. We are not robots. We have emotions. We are humans. This support is really important. Sometimes when you are really frustrated, it is important to realize that there is a person who can help you and that you are not alone in your troubles. The academic load itself can be very heavy and stressful, so consequently, all that 
external support is very important to help international students focus on their program and study.

Participant 4: Back home we didn't interact that much with professors as we do here. They had a scary demeanor. Here there a professors I go out with, play soccer with. Back home I didn't do that. I guess maybe it was because back home I was still young then, in my late-teens. Here, a couple of professors are in my same age group. That might have helped me interact with them. Interactions are much better here.

\section{Theme two: Emotional support and academic success.}

Concerning emotional problems, the participants seemed to shun from topics that deeply explore this category. However, they all agreed that appropriate emotional support would help alleviate those stresses. All the four participants believed that emotional support would significantly affect the achievement of their academic success:

Participant 1: No actually not but I think because I have a very good external emotional support group, if that makes sense. I knew academia was not going to be easy but I didn't really let it affect me emotionally and when I left the classroom I was able to meet with friends who were being supportive like reading the Dominion Post and lets work through the English. I never had any emotional problems. The only thing would be you do miss your family if that counts? Because taking classes in a different language and that stuff. It is hard. Sometimes you wish you were closer to your family but I have to say that the technology elevated it. You can Skype and you can use your phone and you can email and get instant answers instead of having to wait a month until a letter comes. Like in the old, old days. I was appreciative of that. I would have to say no emotional issues except home sickness. That I knew if you go 3 or 4,000 miles away from home 
you are bound to miss your family. Culture wise I can say it was not too different because I am from Germany so we are similar to the U.S. White Caucasian, a lot of traditions they come from Europe are very similar so I didn't have to adjust. I know from other people who come from Asia or Africa, that is a whole different world. So I know their emotional distress was much higher. I did not come straight as a student at WVU. I was only one year in Morgantown. I was able to create friendships, getting to know the program, getting to know a little bit about WVU a year prior so when I came back as a student all I had to focus on was my program and I happened to have a graduate assistantship before working there. But I know a lot of students who come to Morgantown as a student and they deal with a lot more stress. They don't know anybody. They don't know the town. That might had been more difficult and my advantage was I was already here a year so I think sometimes that helped the emotional part. It may have helped the language part a little bit more because you get to practice your conversational English already for a year. It made me a little bit more confident using the language instead of coming straight from Germany studying the doctoral program. I had a year of a cushion. I think that helped a lot.

Participant 2: Yes, but I think that is normal in life. You have trouble with friends and people you are dating, but that is quite normal. I don't think that I have had any significant emotional distress - just the normal stuff. Nothing significant like losing someone or things like that.

Participant 3: I have and it is mostly related to not being able to the readings. It is related to my academic life. Physically I was not able to do the readings on a particular day and I am perfectionist. If the readings are assigned, the professor is getting you ready 
for the class - it is homework. I think there is a cultural aspect as well. It's not related to academics or the university but just being in another culture and finding yourself in a foreign environment. Sometimes you can just get disappointed or frustrated because people see you in a different way while you were expecting a different reaction from them. Being Teaching Assistant, there were some moments of emotional disturbance while I was teaching. I teach Americans, so I question myself whether they could understand me and if I was clear enough with explaining some rules or requirements in their native language. I think this all might go under the emotional category. So not being accepted, being laughed at because of the accent, not being able to understand or explain something.

The participants have received more or less emotional support during their graduate study at WVU, and they described this type of support in different ways:

Participant 2: Usually my friends are my support for things like that. It's good to have friends for a supportive network.

Participant 3: My colleagues, most of them are international students and share our experiences and sometimes those pieces of advice might be very helpful. I have never talked to an authority, I've never talked to the office of Social Justice. I've never gone that far because I didn't have very serious issues like when I was emotionally exhausted and couldn't do anything. Just colleagues and other internationals I would say.

Participant 4: Personally, no. By the time I came to WVU I knew the culture. Clearly I come from a different country but I had been living here for a while. There wasn't that adjustment as if I had come fresh from the plane. I didn't need that support. But I also had a lot of family here at that time. In that sense I would say that I am not your typical 
international student. I had friends that came here straight from my country and they had no one. People like that are better candidates for support systems like that.

However, the participants believed that certain emotional support would help them achieve their academic success more effectively:

Participant 1: Yes. Thinking back on the other question you asked, emotional support for sure helped me with the academic success. It did so in this case because knowing back when I had any problems whatsoever, I knew I could go and have people to talk to. These people were very confident that I could master my education. They always reaffirmed we can get that taken care of or whatever the issue was so once you have the affirmation that people believe in you, it is good. Sometimes I am not sure like when you write a paper and you are not sure, they are always there and they say you can do it. That helps, believing in yourself. I think if you believe in yourself it will affect your academic success. Language wise, that was ok. But if you have believing in you, I do think that. Participant 2: Yes, it is important to have friends that will support you when you need them. I had a very homophobic housemate who said offensive stuff, but my other housemates supported me.

Participant 3: I think it is significantly related. When a person feels good, life is good and our attitude is good meaning we can achieve more and get more in. We are more inspired, enthusiastic, ready to do something, and move forward. But when we are in an emotional hole, emotional disorder, you don't want to do anything and your academic life goes down. The general attitude towards everything we do in life also goes down.

Theme three: Financial support and academic success. 
All the participants expressed more or less financial problems or difficulties. However, they've all received necessary financial support from the university, and they all believed that financial support would significantly affect the achievement of their academic success:

Participant 1: The only thing probably would be when we get tuition waivers as a graduate assistant but then what happens is the fee went up. Like the student fees and the salary never went up proportioned with the increase of the fee. Toward the end of the term it was very hard to like pay $\$ 900$ or $\$ 1000$ to pay for nine credits. That is hard to find $\$ 1,000$ at the beginning of the semester although they do offer you can sign up for a loan. That is why I know and not only me but other international students that I happen to know that this is a burden because a lot of them only have nine months position. For them they have their nine month salary to cover three months of summer and have to find in August another $\$ 1000$ and in January another $\$ 1000$. I knew some of them would love to go home more often but they don't have parents who could pay the airfare. They cannot possibly save the money out of their nine month salary because a doctor's life can be very expensive. I have parents who would pay for this. Within a student budget you wish I could buy this but this is about wants not necessarily what I need so I was ok. I was fortunate to have the twelve month graduate assistantship and that covered me all year around. I didn't have to worry about layoffs where they couldn't continue the GA. I worked in three different offices.

Participant 2: Currently I don't have any money to pay my financial fees. Debt is a problem, I owe the university $\$ 1,400$. I don't see it as a big problem, because I will pay it off by the end of the semester. That's one thing bad about the Computer Science Department because the fees are actually quite high. This semester it was $\$ 1,400$ for one 
semester. We have really high tuition fees.

Participant 3: As I mentioned before, if we had more it would be better. Life is expensive and it is getting more expensive. It also depends on a person's qualities, my mother taught me how to save money and not throw it towards the wind. I would say that the payment that we receive is not enough because half of it is rent. It is not enough for the work we do. Everybody would be happy if it was little bit more. When it comes to money, there is never enough. But with the expenses that every person has, it is not enough especially considering that there is no opportunity to earn another income because there is physically not enough time to work extra hours. We cannot find jobs as internationals because we can only work 20 hours a week. The stipend that we get every two weeks is not enough.

Participant 4: We have all had some money problems, but nothing too serious like bankruptcy or real debt. Once in a while you do spend all your money and wonder where it all went. I went to a school in Boston and my family paid my tuition. Now, being older I figured I had to make my own way and luckily I got an assistantship and I've had that the whole time I've been at WVU.

All the participants have been financially supported in one way or another:

Participant 1: . I probably should say I was very fortunate that I have graduate assistantships at the very beginning. When I came here, I already had a twelve month graduate assistantship so it already covered me all year around. Now you just learn how to adjust within those means you earn although I do have to say that I was fortunate my parents were willing like paying for tickets to come home. I didn't have to take that money out of my expenses. If I wanted to travel they were very helpful in giving me 
money. I am fortunate to have parents that I could rely on. Even if a situation would have happened, I knew I could have called them. And then you learn to live with whatever your monthly income is. I never owned a car because that would have pushed it too high. I could not afford that. You can get a new car but affects how you get around Morgantown because it would have been easier if I had a car but at the same time you have to be financially sensible.

You kind of know at the end and you have to go somewhere else. I think it was before the economy went down. There was somehow less students applying for GA positions though it was possible for everyone but once the economy went down I know that I heard from people like thirty or forty people but now like fifty, sixty people, everyone is looking for getting their tuition taken care of because a lot more applied who usually would have gotten a loan but now don't want to get a loan so it has gotten harder to get a GA position. But during my program there was not a problem at all.

Participant 2: No, because I already get funded as a research assistant or as a teaching assistant. Currently, I'm a teaching assistant because the project that I was working on as a research assistant ended. But I don't think I'll have a problem because if there is no research assistantship, I will always get a teaching assistantship. I have good grades, relations with the students I teach, and recommendations from the professors so it isn't really a problem to get a teaching assistantship. The computer science department is good with that because a lot of the professors do research so they have a lot of funding plus there are a lot classes that they teach. So there are a lot of positions for teaching assistants. They also give priority to Ph.D. students. I think all the Ph.D. students have an assistantship and most of the Masters students. So I think it really depends on the 
department.

Participant 3: I'm an graduate assistant, so my tuition is covered and my insurance is covered. But for the amount of work that has to be done, I would say that the monthly scholarship is not enough. We do a lot of stuff. I wouldn't say there has been a time when I didn't have any money. I would say no.

Participant 4: It's been secure, every once in a while trouble seems to find me. But it's been secure. When I first came in I had a different assistantship, but I had to leave it when my problems started. So this has been my second assistantship at WVU.

All the participants believed that necessary financial support would significantly affect the achievement of their academic success:

Participant 1: I think so because that was actually the only way I would go to a university here because in Germany for the longest time we don't pay any tuition. The government paid for your education and you only paid student fees which were minimal. Like $\$ 100$. They just now, a couple of years ago started to charge no more than $\$ 500$ per semester but still it is cheap compared to here. I always knew when I came here I had to have some kind of financial support because I didn't want to take out a loan so I got that one and by having these GA positions I didn't have to worry how would I pay the next semester. How do I live? It would be a daily struggle if you work at an hourly job and you don't know how many hours they give you to work or you don't know how secure the job is. I never had to deal with these issues and I was pretty sure I didn't have to worry about how to pay for my education. I would not have been this good focusing on my academic success because it takes energy and attention away from me so I didn't have to. 
Participant 2: For sure, because if you can't finance yourself then it will be a big problem. It helps a lot when you don't have to worry about paying your rent or buying food. As a graduate assistant you always get the full tuition waivered, so that's great.

Participant 3: I think yes, because even when you want to buy books for the course they are expensive - and you'll have to share a book. If you wanted to travel to a conference, you cannot afford it because the university does not reimburse it. You have to pay for the ticket, registration, and accommodations. Sometimes the university helps, but it is just a very limited sum of money. I think it impedes academic life. You're a grad student - you are expected to do research. You're a scholar - you cannot be limited to the boundaries of your university. You need some outreach. That is why many people just don't go to conferences because it tends to be unaffordable. I think it is one of the main, not problems, but concerns that I have about the academic life of grad students here. They are not able to go to conferences to learn from others. It is an important part of graduate life.

Participant 4: Yes, it does. If you don't have to worry about your tuition and taking out loans it does help you. There are two sides to it. Some people might say that if you take loans out it might make you more serious. With an assistantship it isn't coming out of your pocket so you are less serious. But with an assistantship you also have to do well academically, so it balances itself out. You don't have to worry about having rent to pay, or find a time to study; it doesn't cut into class time. It provides you with an opportunity to do better, but it depends on the individual and if they take that opportunity.

\section{Theme four: Spiritual support and academic success.}


All the participants had different spiritual experiences. They expressed their spiritual confusion or difficulties in different way. However, they all believed that it is important for international graduate students to feel ease and safe spiritually in order to achieve academic success:

Participant 1: I am Lutheran, Christian basically. I tried for years because I usually go in my home town to a church and I like a minister there and I tried to find something similar here but I have not been able to because I am coming with a certain expectation. How my worship should be like, how I want the minister to be like and I have not been able to find that. But it was not an issue for me because in my opinion spirituality is not defined that I get to go every Sunday to church. It was more defined by that I find good people and I can talk to God anytime because you don't have to have a Sunday worship to talk to him. You can put in a prayer any time you want to and you can seek support any time you want to. I miss the teachings about the Bible. When you read it yourself it is hard sometimes to understand the contexts of these times but lately I found a church and the minister does a great job. It is small. It is denominational and that helps because they accept anybody which I like because in Germany we aren't as structured as they are here. There are many, many subgroups although we all believe in the same being, right but in Germany we are either Catholic or Lutheran. I don't have Baptists, I don't have Methodists. It gets very confusing so I like the denominational church. They do a good job. In the beginning basically it would have been nice to have had a church to go to. I never clicked with them but at the same time that doesn't make me feel less spiritual or was having problems. No because like I said you can talk to God any time. But now I do appreciate the fact that I do have a church again. 
Participant 2: Well, not really because I am an atheist. I have always been an atheist. I have never had to deal with that or issues with that.

That is how they get people to go to church and convert. I don't like that, I've had that problem with the Office of International Students because they are promoting their Christian missions in life and so I reported them to the Social Justice Department because I don't find it nice and that church is not nice at all - The Christian Missionary Alliance. They are very homophobic and practice conversion therapy for gay people, which is very offensive. That is why I have problems with that church. I agree a lot of the churches here are targeting international students and trying to get them to go to their church especially since you feel lonely at the beginning - you don't have friends and you're alone. They try to be your friends in order to get you to go to church.

Most of it is actually how they are just presenting themselves because they want to get the people. If there is something that I don't like about that West Virginia, or I think it is America in general, it is that there are a lot of people that are doing that trying to convert them to their religion.

Maybe it is because you come from a country where the majority of people are atheist. Unlike in my country where the majority is Christian, I grew up knowing Christianity. About 20\% are Muslim, so I've known about that. In middle school I decided I was an atheist. That's why I think I don't have problem is because I've seen the different religions and I've made up my mind that I don't believe in anything and at this point in my life I don't really need it.

I guess because I really believe in science. A lot of the religions all have different interpretations of the Bible, Quran, etc. and yet it is a bunch of stories that don't make 
much sense. It says god created Adam and Eve, but there is the evolution theory. I believe that because there is scientific evidence for it. I don't really like any organized religion because most of the time they are using you for political purposes. For controlling people and that was one of the reasons Christianity became popular in Europe. At the beginning it wasn't accepted. But when one of the kings realized that it was very useful for controlling the people, he converted to Christianity and that is what it started to become popular in Europe. I see organized religion as a way of controlling people. As believing in a higher force, I think that can be helpful for some people if they want to believe. I don't have anything against that.

Participant 3: I haven’t because I am Russian Orthodox, so I don't have to go to church every Sunday. It's my own approach to religion, it's to faith. I'm a believer, but in my own way. Here there is no Orthodox church, I think there is one but it isn't Russian Orthodox, so it's not really my religion or confession. For some people who come from religious environments, like people from the Middle East, it is very important for them. It is a part of their culture and everyday life. They would probably experience a disturbance and problem if they couldn't practice. On my part, I haven't been impacted. If I want to pray, I can pray wherever. Personally, I haven't experienced this. But on Christmas I like going to different churches. I like going to different churches because it is a cultural interest for me. I want to see how people practice it, what traditions they have, and why it is important they do it that way. Behind every religious practice there is culture and it tells me much more about the people I am interacting with and the world I am living in. It broadens my world outlook. So far I have been to Baptist church, Protestant church, Catholic Church, and a Mosque. I was invited during Ramadan, during fasting breaks 
Friday night. I just joined my friend because I am trying to be open to different religions. It's not that I am practicing them, but I just wanted to see. Here people celebrate Christmas on the $25^{\text {th }}$ of December, but in Russia people celebrate it in January. I feel that it is important for me to hear singing and Christmas carols. It's like a Christmas concert for me, not that I am practicing.

Participant 4: I grew up in a very religious family. My mother is still very religious. But the older I got, I just noticed that I became less religious. I am more spiritual than I am religious. People are free to practice their religion - it really doesn't matter to me. I have my own beliefs, but religion just wasn't paramount to me. I think people should do what they feel is best for them as long as they respect others. I go to church when I feel like it, sometimes I do sometimes I don't; before I used to go all the time. Some things at church I don't agree with.

I don't have a religion to criticize. If I was anything I would say I'm Christian. But I have my own issues with Christianity, so if they criticize it, I can't stop them. I think that is what people should do, they should question things. There are things about Islam that I question. I question Judaism. You can't just be gullible and accept everything that is told to you. If something doesn't make sense, you should be able to question it, but that is just my belief.

The participants believed that being spiritually ease and safe is very important for them to achieve academic success:

Participant 1: I don't know if it is directly related. It could indirectly be because if you center it within yourself and you are at peace that perhaps would have an effect of I am more relaxed and therefore if I am more relaxed because I am more at ease and peace in 
myself then I think it may affect. I can say directly as much as it is my emotional support. That is where I think it has a greater impact then the spiritual support. That is interesting because I never thought of it that way. I practice the spiritual support anyway even though I did not have a church so probably I should say perhaps it did. Yes because it kind of centered.

And you could venture the thought that it relates to academic success. Yes. I think yes because I never see it directly. It does. I think so. It helps create a more helpful for seeking a more spiritual environment for you to concentrate on your work and helps you succeed.

Participant 3: It affects, it also addresses personal feelings in those people who don't have the opportunity to practice their religion. I think there is a grain of worry when they cannot do what they are used to doing or culturally supposed to do. It might affect academic life. I think everything affects academic life. If you feel comfortable and safe in a place, if we are emotionally stable, it affects our academic life because it impacts of interactions and approach to different problems. If there is trouble, we cannot read the book, we concentrate on that problem and nothing else. We go unprepared to class and to take the test.

\section{Theme five: Social support and GPA.}

The participants agreed that GPA is a necessary indicator of academic success for international graduate students. However, they argued that there are more important factors that contribute to academic success for graduate study. They believed that all the discussed types of social support may affect the success in GPA:

Participant 1: It should always be like a benchmark. You can always see where you are 
proceeding to. Like I would need some kind of feedback in moving towards am I good. Can I get better and get a lot better whether it be A or A+ or B or B+. Something like that. I want to see yes I am moving in the right direction. However a GPA is not indicative of a lot of skills and traits. You need for yourself need to develop during the course of studies like it is great if you can write papers well but that doesn't mean necessarily, you could problem solving skills. You are a patient person. There are a lot of things a GPA won't address. That just tells me you are good at writing papers or participating in a class. But even that is not a given because sometimes professors allow you to make up a grade if you slacked off or you were lazy and then you were like at the end of the semester can I have extra credit. The professor means well so they give you extra credit but technically what it does, it doesn't tell whoever is looking at the GPA that this person isn't a quick worker, they are procrastinators maybe. Then at the last minute they can fix the problem but in real life if your boss tells you this is due, you better finish the project on time. You don't get an extension. Sometimes the GPA yes it gives me an idea at moving towards something but it needs to have a different type of assessment to find out how rich the community the students really are or how well do they communicate. How much of a self starter are they. How much do they care about other people to make sure they are moving along or are they self centered. These little things. It is a lot to put on an educational plate. I am not sure they will ever be addressed because a lot of factors are not only addressed in the classroom but that would depend on the students, the parents, the support group because if they don't share the same educational values, it might be hard for a student to succeed if you don't have the support. Emotionally, spiritually. The GPA is one way but there should be other ways. 
Participant 2: Yes, it is. If you're capable of getting A's in your classes that means you understand the material and comprehend it well. I guess it also involves good time management to be able to handle all of your projects on time and successfully. So yes, I do think that it is important.

It might not be as important, but I do think that it is still important. Another important part of it is being able to perform research independently - to put effort into that. But I think that those two things complement each other because often the classes you are taking as a grad student a lot of them are elective and you're taking classes that are useful for you research and helps you by taking them. Everything helps you be able to do more research. If you understand it, put in the effort, and you get a low grade it means it will make it more difficult for your research because a lot of times in the end you will have to learn that stuff even if you don't learn it in the class. I think that it is still an important part, not nearly as important as in your undergrad because that is the only thing considered in your undergrad as you don't have research.

Participant 3: That's a very controversial question for me because it is good to maintain 4.0. If you want to go further, they look at your GPA and sometimes grades do not reflect the knowledge you have. If they only thing you do in your life is read books and take tests, but it doesn't tell anything about your strivings and your ability to develop ideas. There are some people who find it uninteresting to go a certain class that is a requirement, yet they are very bright people. They are good in their own field. GPA does not reflect whether you are a successful student or not. There are many factors that affect it. Professors are different. Their approach to teaching is different. If the student finds the connection ok, they will do what the teacher wants the test will be fine and the GPA will 
be high. But there is also the extracurricular work that factors in. It is a very hard question.

To get the Teaching Assistantship, you only need B's and A's. You have to keep your GPA up. If you go to grad school, you are expected to put effort into everything that you do, so it's not that if you have one C that grad school isn't for you. You want to learn something, you want to contribute, and you want to share.

Participant 4: Undergraduate, yes. Graduate, not so much. As an undergraduate if I didn't get an A, or a high B I would be concerned. But now it matters less to me. It's all about the experience and what I get out of it. At some level, especially at the doctorate level, it shouldn't be a GPA factor any more. It should be the quality of work, "did you do it well enough to be satisfactory?" Before being accepted at the doctoral level you should have the quality based on your GPA, but at that point there are other things. Now if I get a B it doesn't bother me as much as it did if I believe I got quality experience from that class. If I got an A and I did not learn anything from that class, that bothers me. I feel how I will be judged with my graduate degree will not be what GPA I have but on my thought process. If I speak intelligently and am analytical with my reasoning and if it makes sense, that will prove the quality of my knowledge. I know people who have a 4.0 GPA and they can't hold a conversation. Let's be honest, there are lots of ways to get an A without being knowledgeable. So I don't really put a lot of emphasis on it anymore. If I were to hire someone I would not look at the GPA. I would sit them down for a half hour discussion and from there I would decide if they are good enough to hire. That's my philosophy.

Experience that you get out of it. If I were teaching at a doctoral program, I probably 
wouldn't give tests or papers. At the end of the course I would sit down one on one with students and discuss things from the class and see how they have evolved it. It could be just on one topic from class. That's what the real world would do. A lot of graduate students work full time. A lot of them may not have the time to do what it takes to get an A, but that doesn't mean that they don't know the stuff. If I have a discussion with you about some issue, I will know for a fact how well you know it. If I give you a paper, how will I know you wrote the paper? Anyone can write a paper. If I sit down with you, I'll learn more about what you know than what any paper could tell me.

However different that the participants have perceived the importance of GPA for graduate study, they all agreed that all those types of social support (Academic support, Emotional support, Financial support and Spiritual support) may affect the achievement of GPA in one way or another:

Participant 1: If any of them affect my GPA, probably I would say the academic support because that would help with the grammar, the writing and things like that because that is always something you can polish and that directly influences the GPA. The question would probably be how would you define academic success because with you separating in with GPA success so success if you want to construed it with a graduate program, I feel like it made me a more well rounded person and I have a GPA I am ok with. Everybody has different expectations about their GPA. Using only the GPA I would say the academic support because that directly translates into a GPA. Emotional support not always translates into a GPA because that just helps me be a good person, not necessarily make me want better. Financial support because I have more peace in my mind to write and not owing money perhaps if that could translate perhaps into a GPA but I think the 
most would be the academic support.

Participant 2: Well, if you don't get any support and you need it, it might affect it negatively. If you don't need support and you get it, it might affect it positively.

Yes, you would have to worry about that too much and get a job that isn't related to your school at all. Maybe you'll end up working at a bar or something that doesn't help you work on your graduate studies.

Participant 3: Yes, of course. Those social supports will affect GPA directly and indirectly.

Participant 4: Yes, it does affect. I don't want to say that it does, it can affect your GPA if you let it. For the financial aspect if you really need money, you'll need two or three jobs and that will impact your GPA. It will affect your ability, especially if you have to write a twenty page paper. If you use my method, through a discussion you might be able to learn more instead of going to look for papers and research articles. If you're dealing with emotional problems, you've never been away from home, you had to leave your country and leave your family, or if you just got married and had kids then had to leave them to go to school, and you take a while to adjust - longer than someone who doesn't have a wife and kids. It might affect your GPA. It's too distractive and you can't concentrate. 


\section{Chapter V}

\section{Discussion}

\section{Summary}

The purpose of this study was to investigate the relationship between the social support perceived by the international graduate students and their academic success in Mid-Atlantic Land Grant Research University. This study conducted multiple regression models to examine the correlation between GPA and social support, Academic success and social support to answer research questions 1 and 2. This study also applied phenomenological research to investigate international graduate students' perception of the relationship between academic success and social support. The goal of this study was to help higher educational administrators and researchers to further understand the problem of international graduate students' adjustment in Mid-Atlantic Land Grant Research University so that more measures would be taken to facilitate international graduate students' adjustment to the new learning and living environment at MidAtlantic Land Grant Research University.

100 international graduate students from five regions (South-East Asia, Middle-East, Europe, Africa, Latin-America) in Mid-Atlantic Land Grant Research University (WVU) participated in the survey titled International Graduate Students Social Support Survey for this research. The list of the contact information of international graduate students was obtained from the Office of International Students and Scholars (OISS). The survey was also delivered to international graduate students during the international activities, events and festivals organized by the Office of International Students and Scholars (OISS).

4 International graduate students (including 2 male and 2 female) from Europe, Africa, Asia and Middle-East countries were interviewed for the qualitative part of this research. Among 
these participants, one is in the first year of a doctoral program in C\&I (Curriculum \& Instruction). She just achieved her master's degree in linguistics at WVU and decided to further her study in a different but related field. One participant is in the second year of a doctoral program in computer science. One is in the third year of a doctoral program in educational technology. The other is in her last year of a doctoral program in EDLS (Educational Leadership Studies). She just found a full-time job at WVU, and in the meantime works on her dissertation.

For the quantitative part of this research (research question one and two), the independent variables (predictors) were Gender, Age, Region, Year, AcademicSupport, EmotionalSupport, FinancialSupport and SpiritualSupport. The dependent variables were GPA and AcademicSuccess.

In order to answer research question one and two, the standard multiple regression analysis was conducted to build up two multiple regression models. The summarization of the findings was listed below.

\section{Findings of research question one}

Research question one stated: Can we predict international graduate students' GPA from measures of gender, age, region, year, academic, emotional, financial and spiritual support?

With six independent variables (Age and Region were deleted from multiple regression model 1 because the results of the Pearson correlation analysis (see Table 4.3) told us that there was no significant correlation between the predictor variable of Age and the outcome variable GPA with $r=.14, p>.05$; and there was also no significant correlation between the predictor variable of Region and the outcome variable GPA with $r=.16, p>.05$.) served as predictors, GPA served as outcome variable, it was found that: (1) the significant regression model to estimate GPA was: 
$\mathrm{GPA}=0.872+0.20$ gender +0.18 year +0.86 academicsupport +0.28 emotionalsupport +0.54 financialsu pport+0.12spiritualsupport $(\mathrm{F}=30.56, p<.01)$; (2) the most significant predictor to estimate GPA was Academic Support $(\beta=.45, p<.01)$, followed by Financial Support $(\beta=.30, p<.05)$, Year $(\beta=.28, p<.05)$, Emotional Support $(\beta=. .26, p<.05)$, Gender $(\beta=.18, p<.05)$ and Spiritual Support $(\beta=.12, p<.05) ;(3)$ other two independent variables (Age and Region) were not significantly associated with GPA.

Academic support. The implication of the finding that the predictor of Academic Support had a big size of regression correlation with the outcome variable GPA, with $\beta=.45, \mathrm{t}$ $(100)=4.889, p<.01$ was that academic support would significantly affect international graduate students' achievement of GPA. The more academic support international graduate students receive from the university, the higher GPA they may achieve. On the contrary, the less academic support international graduate students receive from the university, the lower GPA they may achieve. This indicated that the lack of essential academic support may significantly affect the achievement of international graduate students' GPA.

Financial support. The implication of the finding that the predictor of Financial Support had a small size of regression correlation with the outcome variable GPA, with $\beta=.30, \mathrm{t}(100)=$ $3.822, p<.05$ was that financial support would significantly affect international graduate students' achievement of GPA. However, the regression correlation between predictor variable Financial Support and outcome variable GPA was relatively small. This indicated that the lack of financial support may significantly but not necessarily affect the achievement of international graduate students' GPA.

Year. After the second operation of multiple regression model, the predictor of Year had a small size of regression correlation with the outcome variable GPA, with $\beta=.28, \mathrm{t}(100)=$ 
$3.745, p<.05$. This indicated that in different school year, the achievement of international graduate students' GPA may be different. Take the researcher herself for example, in the later years she obtained higher GPA than in the earlier years. This made sense at one point that international graduate students need time to adjust to the new learning and living environment, and this may affect the achievement of international graduate students' GPA.

Emotional support. The implication of the finding that the predictor of Emotional Support had a small size of regression correlation with the outcome variable GPA, with $\beta=.26, \mathrm{t}$ $(100)=3.609, p<.05$ was that emotional support would significantly affect international graduate students' achievement of GPA. However, the regression correlation between predictor variable emotional support and outcome variable GPA was relatively small. This indicated that the lack of financial support may significantly but not necessarily affect the achievement of international graduate students' GPA.

Gender. The implication of the finding that the predictor of Gender had a small size of regression correlation with the outcome variable GPA, with $\beta=.18, \mathrm{t}(100)=0.822, p<.05$ was that gender would significantly affect international graduate students' achievement of GPA. In this study, the researcher found out that female international graduate students had relatively higher GPA than male international graduate students. However, the regression correlation between predictor variable Gender and outcome variable GPA was very small. This indicated that the lack of financial support may significantly but not necessarily affect the achievement of international graduate students' GPA.

Spiritual support. The implication of the finding that the predictor of Spiritual Support had a small size of regression correlation with the outcome variable GPA, with $\beta=.12, \mathrm{t}(100)$ $=0.904, p<.05$ was that spiritual support would significantly affect international graduate 
students' achievement of GPA. However, there was a very small size of regression correlation between predictor variable Spiritual Support and outcome variable GPA. This indicated that the lack of financial support may significantly but not necessarily affect the achievement of international graduate students' GPA.

\section{Findings of research question two}

Research question two stated: Can we predict international graduate students' academic success from measures of gender, age, region, year, academic, emotional, financial and spiritual support?

With eight independent variables served as predictors, AcademicSuccess served as outcome variable, it was found that: (1) the significant regression model to estimate AcademicSuccess was:

AcademicSuccess $=0.967+0.28$ gender +0.14 age +0.17 region +0.26 year +0.89 academicsupport +0.3 6emotionalsupport+0.48financialsupport+0.26spiritualsupport $(\mathrm{F}=32.056, p<.01)$; (2) the most significant predictor to estimate Academic Success was Academic Support $(\beta=.56, p<.01)$, followed by Financial Support $(\beta=.28, p<.01)$, Emotional Support $(\beta=.24, p<.01)$, Year $(\beta=.19$, $p<.05)$, Region $(\beta=.19, p<.05)$, Gender $(\beta=.14, p<.05)$, Age $(\beta=.10, p<.05)$ and Spiritual Support $(\beta=.10, p<.01) .(3)$ however, the predictors of Gender, Age and Region had a significant regression correlation with the outcome variable AcademicSuccess only after the second operation of multiple regression model 2.

Academic support. The finding that the predictor of Academic Support had a big size of regression correlation with the outcome variable AcademicSuccess, with $\beta=.56, p<.01$ indicated that international graduate students' academic success would be significantly influenced by academic support. If international graduate students didn't receive sufficient support concerning 
course registration, writing skill improvement, academic advice and so forth, they might encounter significant difficulties on their way to achieve academic success. This indicated that the lack of essential academic support may significantly affect the achievement of international graduate students' academic success.

Financial support. The finding that the predictor of Financial Support had a small size of regression correlation with the outcome variable AcademicSuccess, with $\beta=.28, p<.01$ indicated that financial support would significantly affect international graduate students' achievement of academic success. If there were not sufficient support for international graduate students involving the aspects of tuition waiver, graduate assistantship, part-time jobs and so forth, it might be very difficult and distractive for international graduate students to achieve their academic success. However, the regression correlation between predictor variable Financial Support and outcome variable AvademicSuccess was relatively small. This indicated that the lack of financial support may significantly but not necessarily affect the achievement of international graduate students' academic success.

Emotional support. The finding that the predictor of Emotional Support had a small size of regression correlation with the outcome variable AcademicSuccess, with $\beta=.24, p<.01$ indicated that emotional support would significantly affect international graduate students' achievement of academic success. However, there was a relatively small regression correlation between the predictor variable of emotional Support and the outcome variable of AcademicSuccess. This indicated that the lack of emotional support may significantly but not necessarily affect the achievement of international graduate students' academic success.

Year. The finding that the predictor of Year had a small size of regression correlation with the outcome variable AcademicSuccess, with $\beta=.19, p<.05$ indicated that in different school 
year, international graduate students may achieve their academic success differently. This is possible because in different school years there may be different factors that affect international graduate students' achievement of academic success. However, the regression correlation between predictor variable emotional Support and outcome variable AcademicSuccess was very small. This indicated that the predictor of Year may significantly but not necessarily affect the achievement of international graduate students' academic success.

Region. The predictor of Region had a small size of regression correlation with the outcome variable AcademicSuccess, with $\beta=.19, p<.05$ only after the second operation of multiple regression model. This indicated international graduate students from the five regions (South-East Asia, Middle-East, Europe, Africa and Latin-America) perceived the achievement of their academic success differently. In different region, the achievement of international graduate students' academic success may be different. However, the regression correlation between the predictor variable Region and the outcome variable AcademicSuccess was very small. This indicated that the predictor of Region may significantly but not necessarily affect the achievement of international graduate students' academic success.

Gender. The finding that the predictor of Gender had a small size of regression correlation with the outcome variable AcademicSuccess, with $\beta=.14, p<.05$ indicated that gender would significantly affect international graduate students' achievement of academic success. In this study, the researcher found out that female international graduate students had more positively perceived their academic success than male international graduate students. However, the regression correlation between predictor variable Gender and outcome variable AcademicSuccess was very small. This indicated that the predictor of Gender may significantly but not necessarily affect the achievement of international graduate students' academic success. 
Age. The implication of the finding that the predictor of Age had a small size of regression correlation with the outcome variable AcademicSuccess, with $\beta=.10, p<.05$ was that age would significantly affect international graduate students' achievement of academic success. In this study, the researcher found out that older international graduate students tended to be more positive than younger international graduate students when they perceived the achievement of their academic success. However, the regression correlation between predictor variable Age and outcome variable AcademicSuccess was very small. This indicated that the predictor of Age may significantly but not necessarily affect the achievement of international graduate students' academic success.

Spiritual support. The finding that the predictor of Spiritual Support had a small size of regression correlation with the outcome variable AcademicSuccess, with $\beta=.10, p<.01$ indicated that spiritual support would significantly affect international graduate students' achievement of academic success. Although spiritual support was not necessarily a decisive factor in the achievement of international graduate students' academic success, international graduate students did report that spiritual support may influence their academic success. However, the regression correlation between predictor variable Spiritual Support and outcome variable AcademicSuccess was very small. This indicated that the lack of spiritual support may significantly but not necessarily affect the achievement of international graduate students' academic success.

\section{Findings of research question three}

Research question three stated: How do international graduate students perceive their academic success and social support system at Mid-Atlantic Land Grant Research University? 
Based on research question three, interview questions were designed to investigate international graduate students' (2 male and 2 female from Europe, Africa, Asia and LatinAmerica respectively) perception of the relationship between social support and academic success when they study at Mid-Atlantic Land Grant Research University. The findings were listed in the table below:

Table 5.1

Development of Themes

\begin{tabular}{|c|c|c|}
\hline Transcribed Interviews & Themes & Findings \\
\hline Writing & Academic Support and & All the participants expressed \\
\hline Large Workload & Academic Success & that necessary academic \\
\hline Expectation of Professors & & support would help alleviate \\
\hline New Learning Style & & academic stress; \\
\hline English Skills & & All the participants believed \\
\hline Research & & that academic support would \\
\hline Dissertation & & significantly affect the \\
\hline Acquiring New Information & & achievement of their academic \\
\hline Full Load of Courses & & success. \\
\hline Talking to Friends and & & \\
\hline Meeting People & & \\
\hline International Students and & & \\
\hline Scholars Office & & \\
\hline Academic Department & & \\
\hline Feedback & & \\
\hline
\end{tabular}




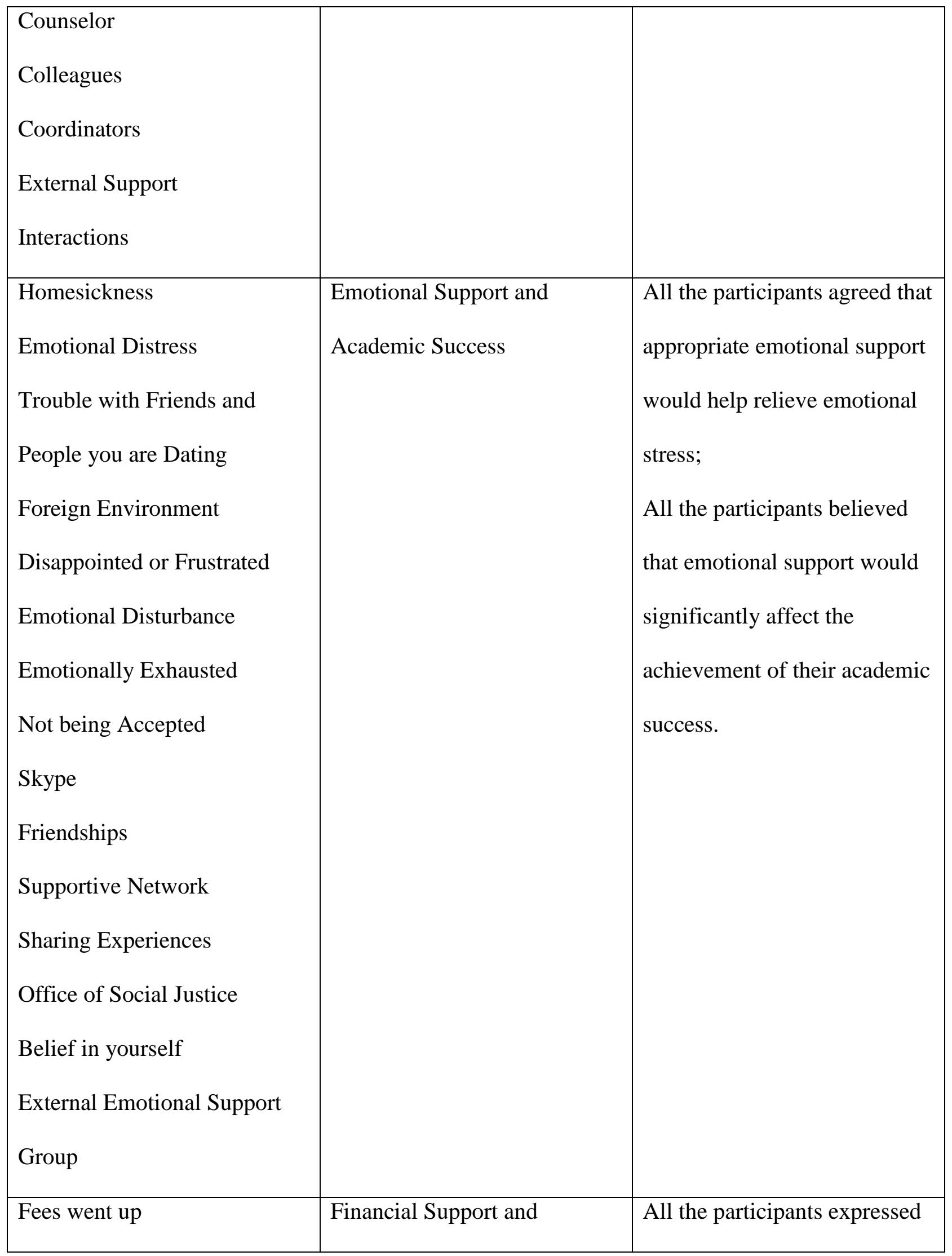




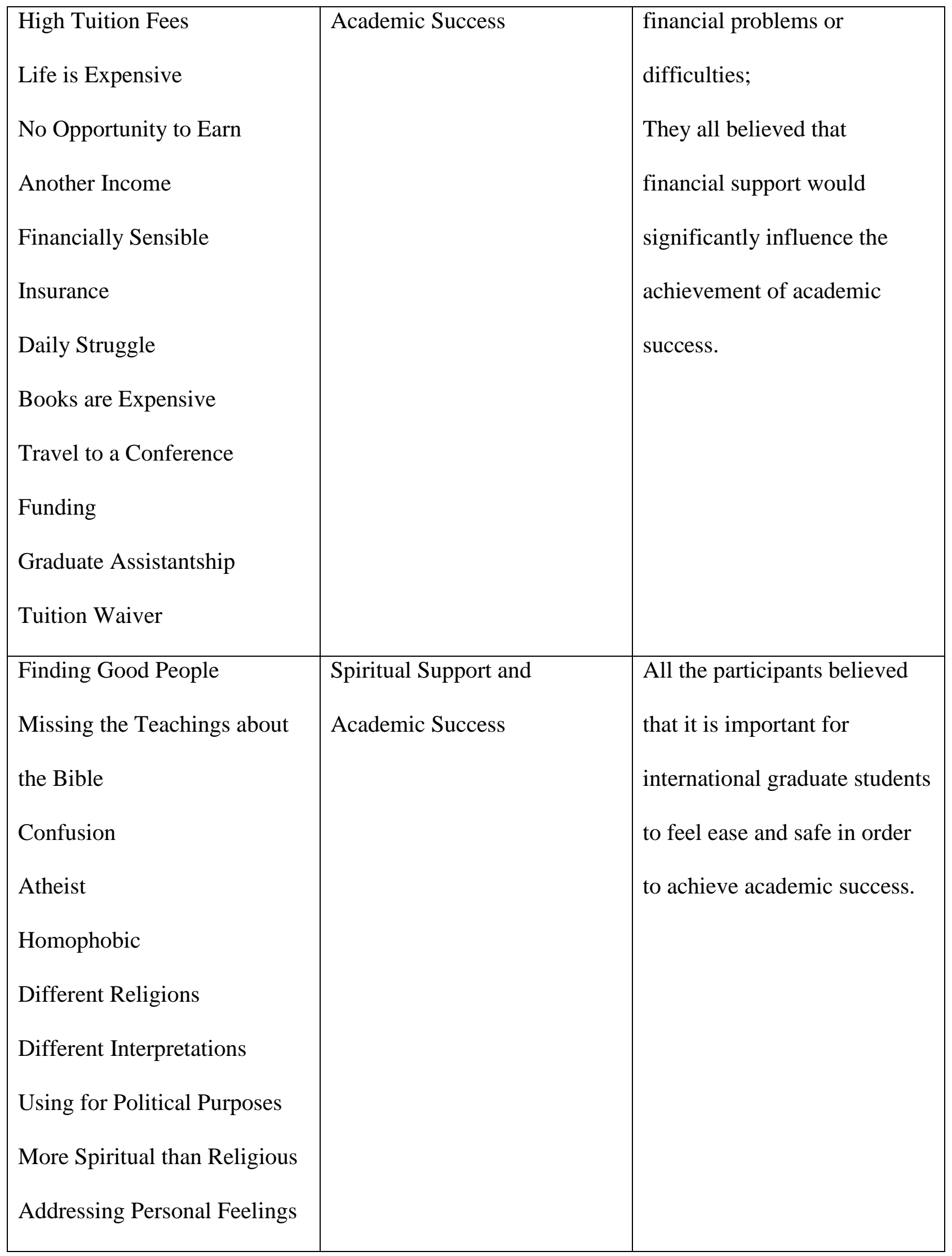




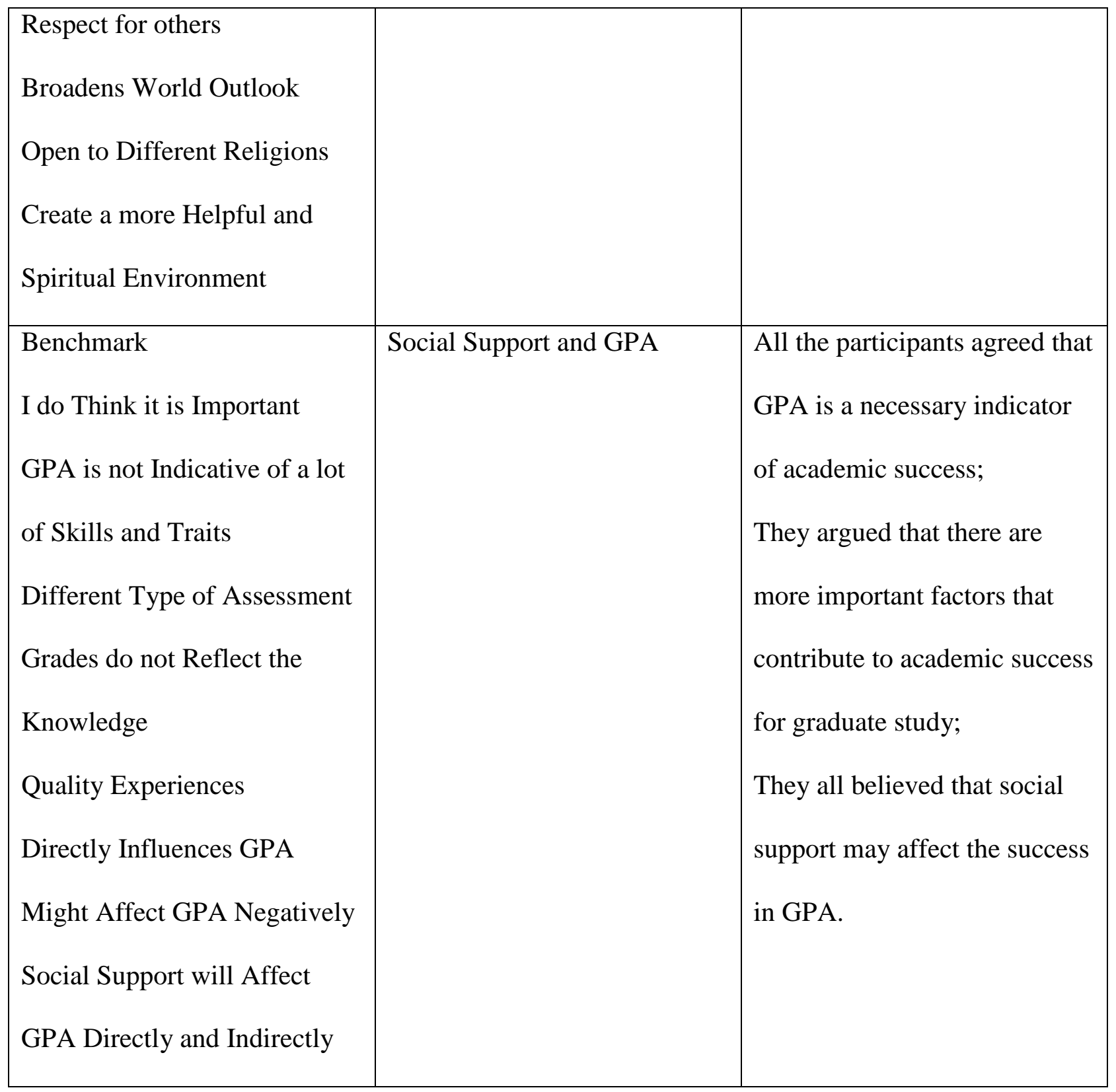

\section{Conclusions}

This study has examined the relationship between social support and academic success for international graduate students at Mid-Atlantic Land Grant Research University through quantitative and qualitative research methods. For research questions one and two, multiple regression models were established to assess the correlations between predictor variables 
(Gender, Age, Region, Year, Academic support, Emotional support, Financial support and Spiritual support) and outcome variables (GPA and Academic success). For research question three, phenomenological research method was conducted to investigate the relationship between social support and academic success for international graduate students.

A number of conclusions were listed below according to the results of the data analysis:

1. The significant multiple regression model to predict GPA is: $\mathrm{GPA}=0.872+0.20$ gender +0.18 year +0.86 academicsupport +0.28 emotionalsupport +0.5 4financialsupport+0.12spiritualsupport.

2. The most significant predictor to estimate GPA is Academic Support, followed by Financial Support, Year, Emotional Support, Gender and Spiritual Support. Other two independent variables (Age and Region) are not significantly associated with GPA.

3. The significant multiple regression model to predict Academic success is: AcademicSuccess $=0.967+0.28$ gender +0.14 age +0.17 region +0.26 year +0.89 academics upport+0.36emotionalsupport+0.48financialsupport+0.26spiritualsupport.

4. The most significant predictor to estimate Academic Success is Academic Support, followed by Financial Support, Emotional Support, Year, Region, Gender, Age and Spiritual Support.

5. All the participants in this study for the qualitative part believe that different levels of social support including academic support, emotional support, financial support and spiritual support will significantly affect the success in GPA and academic achievement for international graduate students directly or indirectly.

\section{Recommendations}

\section{Recommendations for future studies.}


1. This study was applied through a relatively small sample size (the quantitative part involved 100 international graduate students from five different areas; the qualitative part involved 4 international graduate students from four different areas). Future studies may include bigger sample size either through quantitative or qualitative research methods in order to examine the relationship between social support and academic success more accurately.

2. This study was limited to only one institution (Mid-Atlantic Land Grant Research University). Future study may include different higher educational institutions to see if different patterns will come out related with the relationship between social support and academic success for international graduate students.

3. Future studies that consider applying statistical research methods may include different approaches besides multiple regression model. In this study, it was found out that some predictor variables had a significant Pearson correlation with the outcome variable, but no significant regression correlations with the outcome variables. Thus different statistical approaches may yield different results.

\section{Recommendations for college and university administrators.}

1. Higher educational institutions' leaders and administrators should consider all the factors including gender, age, region, year, academic support, emotional support, financial support and spiritual support when they attempt to facilitate international graduate students' academic success.

2. Higher educational institutions' leaders and administrators should keep in mind that results obtained from this study may be helpful to understand their institution's 
current situation about international graduate students' academic success, but not to judge the success or failure in this aspect. 


\section{References}

Al-Jenaibi, B. (2011). The practice of public relations departmets in increasing social support in the diverse workplaces of the United Arab emirates. Cross-Cultural Communication, 7, 41-54.

Altbach, P. (1998). Comparative Higher Education : Knowledge, the University, and Development. Greenwich, CT : Ablex Publishing Corporation.

Ashforth, B.E. \& Mael, F. (1989). Social identity theory and the organization. The Academy of Management Review, 14, 20-39.

Baqutayan, S. (2011). Stress and social support. Indian Journal of Psychological Medicine, 33, 29-34.

Bartell, M. (2003). Internationalization of universities: A university culture-based framework. Higher Education, 45, 43-70.

Bauman, Z. (1999). Culture as Praxis. London: Sage.

Berkman, L.F., Glass, T., Brissette, I. \& Seeman, T.E. (2000). From social integration to health: Durkheim in the new millennium. Social Science \& Medicine, 51, 843-857.

Blau, P.M. (1960). A theory of social integration. American Journal of Sociology, 65, 545-556.

Bochner, S. (1982). The social psychology of cross-cultural relations. In S. Bochner (Ed.), Cultures in contact: Studies in cross-cultural interactions. London: Pergamon.sn.

Bowlby, J. (1969). Attachment and Loss. London: Hogarth Press.

Bowlby, J. (1979). The Making and Breaking of Affectional Bonds. New York: Tavistock Publications.

Bresnahan, C.G. \& Mitroff, I.I. (2007). Leadership and attachment theory. American Psychologist, 25, 607-608. 
Brown, L. (2009). International students in England: Finding belonging through Islam. Journal of Muslim Minority Affairs, 29, 57-67.

Carr, J. L., Koyama, M \& Thiagarajan, M. (2003). A women's support group for Asian international students. Journal of American College Health, 52, 131-134.

Chang, M.J. (2001). The positive educational effects of racial diversity on campus. In G. Orfield and M. Kurlaender (Eds.), Diversity challenged: Evidence on the impact of affirmative action, (pp. 175-186). Cambridge, MA: Harvard Education Publishing Group.

Chao, R.C. (2011). Managing stress and maintaining well-being: Social support, problemfocused coping and avoidant coping. Journal of Counseling \& Development, 89, 338348.

Chen, Y. \& Li, S.X. (2009). Group identity and social preferences. American Economic Review, 99, 431-457.

Cohen, S. (2004). Social relationships and health. American Psychologist, 20, 676-684.

Cohen, S. (2004). The Pittsburgh Common Cold Studies: Psychosocial predictors of susceptibility to respiratory infectious illness. Keynote Presentation at the 8th International Congress of Behavioral Medicine; Mainz, Germany, August 25-28, 2004. Department of Psychology. Paper 274. http://repository.cmu.edu/psychology/274

Cohen, S. \& Mckay, G. (1984). Social support, stress and the buffering hypothesis: A theoretical analysis. In A. Baum, S.E. Taylor \& J.E. singer (Eds.), Handbook of Psychology and Health (pp. 253-267). Hillsdale, NJ: Lawrence Erlbaum Associates.

Cohen, S. \& Wills, T.A. (1985). Stress, social support and buffering hypothesis. Psychological Bulletin, 98, 310-357. 
Connors, M.E. (2011). Attachment theory: A "secure base" for psychotherapy integration. Journal of Psychotherapy Integration, 21, 348-362.

Dao, T.K., Lee, D \& Huang, L. C. (2006). Acculturation level, perceived English fluency, perceived social support level and depression among Taiwanese international students. Higher Education, 35, 56-68.

Durkheim, E. (1951). Suicide. New York: Free Press. (Original work published in 1897)

Ellingboe, B.J. (1998). Divisional strategies to internationalize a campus portrait: Results, resistance, and recommendations from a case study at a U.S. university. In Mestenhauser, J.A. and Ellingboe, B.J. (Eds), Reforming in Higher Education Curriculum: Internationalizing the Campus. Phoenix, AZ: American Council on Education and Oryx Press.

Featherston, M. (1990). Global culture: An introduction. Theory, Culture and Society, 7, 1-14.

Feizi, K. (1990). The correlation between academic success and problems perceived by international graduate students. University of San Francisco.

Fernandez, M.S. (1988). Issues in counseling Southeast-Asian students. Journal of Multicultural Counseling and Development, 16, 157-166.

Field, A. (2009). Discovering Statistics Using SPSS. London: SAGE Publications Ltd.

Fife, J., Adegoke, A., Mccoy, J. \& Brewer, T. (2011). Religious commitment, social support and life satisfaction among college students. College Student Journal, 45, 393-400.

Fletcher, J.F. \& Stren, R.E. (1989). Language skills and adaptation: A study of foreign students in a Canadian university. Curriculum Inquiry, 19, 293-308. 
Frey, L. L. \& Roysircar, G. (2006). South Asian and East Asian international students' perceived prejudice, acculturation, and frequency of help resource utilization. Multicultural Counseling and Development, 34, 208-222.

Furnham, A. (2004). Foreign students: Education and culture shock. Psychologist, 17, 16-19.

Furnham, A. \& Bochner, S. (1986). Culture Shock: Psychological Reactions to Unfamiliar Environments. London: Routledge.

Garber, J. \& Seligman, M.E.P. (1980). Human helplessness: Theory and applications. New York: Academic Press.

Goldlust, J. \& Richmond, A.H. (1974). A multivariate model of immigrant adaptation. International Migration Review, 8, 193-224.

Gumport, P. J. \& Sporn, B. (1999). Institutional adaptation: Demands for management reform and university administration. Higher Education: Handbook of Theory and Research, 14, 103-145.

Habu, T. (2000). The irony of globalization: The experience of Japanese women in British higher education. Higher Education, 39, 43-66.

Hardy, C.L. (2006). Theory of social integration. Retrieved from http://www.ehow.com/about5399164 theory-socail-integration.html\#ixzz1mCJkC5Rv.

Harris, R. (1995). Overseas students in the United Kingdom university system. Higher Education, 29, 77-92.

Hind, D. and Moss, S. (2011). Employability Skills. 2nd Edition. Sunderland, Business Education Publishers.

Hlebec, V., Kogovsek, T. \& Ferligoj, A. (2011). The influence of social support and personal networks on doctoral student performance. Metodoloski zvezki, 8, 157-171. 
Huntington, S. (1993). The clash of civilizations? Foreign Affairs, 72, 3.

Institute of International Education. (2004). Open doors 2004: Report on international educational exchange. New York: Author.

Kagan, H. \& Cohen, J. (1990). Cultural adjustment of international students. Psychological Science, 1, 133-137.

Klineberg, I. (1980). Stressful experiences of foreign students at various stages of sojourn: Counseling and policy implications. In C. V. Coelho and P. I. Ahmed (Eds.), Uprooting and Development Dilemmas of Coping with Modernization. New York: Plenum Press.

Lakey, B., \& Cohen, S. (2000). Social support theory and selecting measures of social support. In S. Cohen, L.U. Gordon \& B.H. Gottlieb (Eds.). Social Support Measurement and Interventions: A Guide for Health and Social Scientists. New York: Oxford.

Lazarus, R.S. (1976). Patterns of Adjustment. New York: McGraw-Hill.

Lazarus, R.S. (1981). The stress and coping paradigm. In Eisdorfer, Cohen, Kleinman and Maxim (Eds.), Theoretical Bases for Psychopathology. New York: Spectrum.

Lazarus, R.S. \& Cohen, J. (1977). Environmental stress. In J. Altman and J. Wohlwell (Eds.), Human Behavior and Environment: Advances in Theory and Research. New York: Plenum Press.

Lazarus, R.S. \& Folkman, S. (1984). Stress, Appraisal and Coping. New York, NY: Springer. Lee, M.Y., Abd-Ella, M. \& Burks, L.A. (1981). Needs of Foreign Students from Developing Nations at U.S. Colleges and Universities. Washington, DC: National Association of Foreign Student Affairs. 
Liebler, G. \& Sandefur, C. (1998). Exchanging social support with friends, neighbors and coworkers. Retrieved on May 14, 2010 from http:// www.ssc.wisc.edu/cde/cdewp/9819.pdf.

Loewenberg, P. (1969). Emotional problems of graduate education. The Journal of Higher Education, 40, 610-623.

Mallinckrodt, B. \& Leong, F. T. (1992). Social support in academic programs and family environments: Sex differences and for conflicts for graduate students. Journal of Counseling \& Development, 70, 716-723.

Maulik, P.K., Eaton, W.W. \& Bradshaw, C.P. (2010). The effect of social networks and social support on mental health services use, following a life event, among the Baltimore epidemiologic catchment area cohort. Journal of Behavioral Health Services \& Research, 6, 29-50.

McClure, J.W. (2007). International graduates' cross-cultural adjustment: Experiences, coping strategies, and suggested programmatic responses. Teaching in Higher Education, 12, $199-217$.

McKinlay, N. J., Pattison, H. M. \& Gross, H. (1996). An exploratory investigation of the effects of a cultural orientation program on the psychological well-being of international university students. Higher Education, 31, 379-395.

Mcleod, S.A. (2008). Simply Psychology; Social Identity Theory. Retrieved from http://www.simplypsychology.org/social-identity-theory.html.

Mendoza, R.H. \& Martinez, J.L. (1981). The measurement of acculturation. In A. Baron Jr. (Ed.), Explorations in Chicano Psychology (pp. 71-82). New York: Praeger. 
Milem, J. (2003). The educational benefits of diversity: Evidence from multiple sectors. In M.J. Chang, D. Witt, J. Jones and K. Hakuta (Eds.), Compelling interest: Examining the evidence on racial dynamics in colleges and universities, (pp. 126-169). Stanford: Stanford University Press.

Olmedo, E.L. (1979). Acculturation: A psychometric perspective. American Psychologist, 34, 1061-1070.

Ozkahraman, S., Yildirim, B. \& Altun, O.S. (2011). Perceived social support level and related factors in Turkish university students. Health MED, 5, 1033-1037.

Perrucci, R.\& Hu, H. (1995). Satisfaction with social and educational experiences among international graduate students. Research in Higher Education, 36, 491-508.

Pistole, M.C. (1989). Attachment: Implications for counselors. Journal of Counseling and Development, 68, 190-193.

Prevatt, F., Li, H. J., Welles, T., Festa-Dreher, D., Yelland, S. \& Lee, J. (2011). The academic success inventory for college students: Scale development and practical implications for use with students. Journal of College Admission, 211, 26-31.

Prior, V. \& Glaser, D. (2006). Understanding Attachment and Attachment Disorders: Theory, Evidence and Practice. London and Philadelphia: Jessica Kingsley Publishers.

Rahman, O. \& Rollock, D. (2004). Acculturation competence and mental health among south Asian students in the United States. Journal of Multicultural Counseling and Development, 32, 130-142.

Riggs, S.A., Jacobvitz, D. \& Hazen, N. (2002). Adult attachment and history of psychotherapy in a normative sample. Psychotherapy: Theory/Research/Practice/Training, 35, 604-625., 
Rosenthal, D.A. \& Hrynevich, C. (1985). Ethnicity and ethnic identity: A comparative study of Greek, Italian and Anglo-Australian adolescents. International Journal of Psychology, 20, 723-742.

Rose-Redwood, C. R. (2010). The challenge of fostering cross-cultural interactions: A case study of international graduate students' perceptions of diversity initiatives. College Student Journal, 44, 23-34.

Rosenthal, L., London, B., Levy, S.R. \& Lobel, M. (2011). The roles of perceived identity compatibility and social support for women in a single-sex STEM program at a coeducational university. Sex Roles, 65, 725-736.

Rust, R. (1960). Epidemiology of mental health in college. Journal of Psychology, 49, 235-248.

Salvini, A. (2010). Symbolic interactionism and social network analysis: An uncertain encounter. Symbolic Interaction, 33, 364-388.

Sam, D. L. (2001). Satisfaction with life among international students: An exploratory study. Social Indicators Research, 53, 315-337.

Sarkodie-Mensah, K. (1998). International students in the U.S. : Trends, cultural adjustment, and solutions for a better experience. Journal of Education for Library and Information Science, 39, 214-222.

Smith, K. P. \& Christakis, N. A. (2008). Social networks and health. The Annual Review of Sociology, 34, 405-429.

Social Support.(2004). In Encyclopedia of Applied Psychology. Retrieved from http://www.credoreference.com/entry/estappliedpsyc/social_support

Speck, M. \& Knipe, C. (2005). Why can't we get it right? Designing high-quality professional development for standards-based schools (2nd ed.). Thousand Oaks: Corwin Press. 
Storti, C. (1990). The art of crossing cultures. Yarmouth, Maine: Intercultural Press.

Swagler, M.A. \& Ellis, M. V. (2003). Crossing the distance: Adjustment of Taiwanese graduate students in the United States. Journal of Counseling Psychology, 50, 420-437.

Tajfel, H. (1978). Differentiation between Social Groups. London: Academic Press.

Tajfel, H. \& Turner, J. (1979). An integrative theory of intergroup conflict. In S. Worchel \& W. Austin (Eds.), The Social Psychology of Intergroup Relations. Monterey, CA: Brooks/Cole.

The Office of International Students and Scholars, WVU (2012). http://oiss.wvu.edu, 2012 Trepte, S. (2006). Social identity theory. In J. Bryant \& P. Vorderer (Eds.), Psychology of Entertainment (pp 255-271). Mahwah, NJ: Lawrence Erlbaum Associations Publishers.

Verheijden, M.W., Bakx, J.C., Van Weel, C., Koelen, M.A. \& Van Staveren, W.A. (2005). Role of social support in lifestyle focused weight management interventions. European Journal of Clinical Nutrition, 59, 179-186.

Volet, S. E. \& Ang, G. (1998). Culturally mixed groups on international campuses: An opportunity for inter-cultural learning. Higher Education Research and Development, 17, $5-23$.

Walton, B. (1968). Foreign students in perspectives. International Education and Culture Exchange, 3, 55-60.

Wan, T., Chapman, D.W. \& Biggs, D.A. (1992). Academic stress of international students attending U.S. universities. Research in Higher Education, 33, 607-623.

Ward, C. \& Kennedy, A. (1996). Crossing cultures: The relationship between psychological and socio-cultural dimensions of cross-cultural adjustment. In Pandey Sinha, J. D. and Bhawuk, D. (Eds), Asian Contributions to Cross-Cultural Psychology. New Delhi: Sage. 
White, A.J., Brown, S.E. \& Suddick, D. (1983). Academic factors affecting the scholastic performance of international students. College Student Journal, 17, 268-272.

Wilks, S.E. \& Spivey, C.A. (2010). Resilience in undergraduate social network students: Social support and adjustment to academic stress. Social Work Education, 29, 276-288.

Wimberley, D.W., McCloud, D.G. \& Flinn, W.L. (1992). Predicting success of Indonesian graduate students in the United States. Comparative Education Review, 36, 487-508.

Wiseman, H. (1997). Far away from home: the loneliness experience of overseas students. Journal of Social and Clinical Psychology, 16, 277-298.

Yang, B. \& Clum, G. A. (1995). Measures of life stress and social support specific to an Asian student population. Journal of Psychopathology \& Behavioral Assessment, 17, 51-67.

Yildirim, I. (1998). Akademik basari duzeyleri farkli olan lise ogrencilerinin sosyal destek duzeyleri. Psikolojik Danisma Ve Rehberlik Dergisi, 2, 33-38.

Ying, Y. \& L. H. Liese. (1991). Emotional well-being of Taiwan foreign students in the US: An examination of pre- to post-arrival differential. International Journal of Intercultural Relations, 15, 345-366.

Ying, Y. W. (2005). Variation in acculturative stressors over time: A study of Taiwanese students in the United States. Journal of Intercultural Relations, 29, 59-71. 
Appendices A,B,C, D, E. 


\section{Appendix A}

\section{International graduate students' cover letter}

April, 2012

Address

City, State, Postal Code

Dear international graduate student (Last Name)

Social support is considered to very important predictor of international graduate students' academic success. However very limited research has been done to measure the correlation between international graduate students' perceived social support and perceived academic success. Therefore, I'm requesting your participating in this research concerning international graduate students' academic success in Mid-Atlantic Land Grant Research University.

Enclosed is a brief international graduate student social support survey. It will take approximately ten minutes of your time to complete. The survey will be submitted to the Institution Review Board (IRB) regarding the protection of human subjects. Hence, all names of participants will remain anonymous in the final research report. If you decide to participate in this research, please respond to the questionnaire in two weeks from the day you receive the survey. If you decide not to participate, please email me at phe1@ mix.wvu.edu. If you have any question, please feel free to contact me with this email address.

I appreciate your time and cooperation.

Best Regards 
Sincerely,

Ping He

Doctoral Candidate

Educational Leadership Studies 


\section{Appendix B}

\section{International Graduate Students’' Reminder}

May, 2012

Dear international graduate student (Last Name),

This is a reminder of the international graduate student social support survey that was sent to you approximately two weeks ago. If you have already responded to the survey, a lot of thanks to you. If you have not, I will really appreciate your feedback which is essential to the completion of this research.

Please feel free to contact by at phe1@mix.wvu.edu or you can also reach me by phone at (304)-906-8062.

Thank you very much for your cooperation.

Best Regards

Sincerely

Ping $\mathrm{He}$

Doctoral Candidate

Educational leadership studies

Mid-Atlantic Land Grant Research University 


\section{Appendix C}

Survey Instrument

\section{International Graduate Students Social Support Survey}

Please read these directions carefully:

You are not being tested. There are no right or wrong answers. This is a list of statements about situations that occasionally trouble (perturb, distress, grieve, annoy or worry) students from other countries who are attending colleges in the United States. The statements are related to areas of admissions, academic work, language, religion, and so forth.

Please follow these directions:

Read the questions carefully, pause at each question, choose the answer that you think best describes your situation.

Demographic Information

1. What is your gender?

Female

C Male

2. How old are you? 
Less than 25

25 to 35

C 35 to 40

c. More than 40

3. Which area are you from?

South-East Asia

Middle-east

C Europe

(C) Africa

Latin-America

4. How many years have you been in U.S.?

\begin{tabular}{l|l}
$\mathbf{C}$ & Less than 1 year \\
$\mathbf{C}$ & 1 to 2 years \\
$\mathbf{C}$ & 3 to 5 years \\
& More than 5 years
\end{tabular}

5. To the best of your knowledge, what is your current grade point average (GPA)?

2.5 to 3.0

C 3.0 to 3.5

3.5 to 4.0

C. 4.0

\section{Perceived Social Support}

On the 1 to 5 point scale, please choose the number that best describes your level of agreement or disagreement with the following statements. 
1=Strongly disagree, 2=Disagree, 3=Neutral, 4=Agree, 5=Strongly agree

\section{Academic Support}

1. I find it easy to register for my courses through WVU STAR system.

12345

2. There' s ample support for me to enhance my English language skills in WVU.

12345

3. I have not been able to find an international support group to talk about my exam anxiety. 12345

4. Because English is my second language, I have a concern about my writing ability.

12345

5. The academic advising for international Graduate students is excellent in WVU. 12345

\section{Emotional Support}

1. I do not think that WVU has considered the emotional support that international graduate students would need.

12345

2. Because of the cultural differences, many international graduate students would feel inferior to others.

12345 
3. WVU allows international graduate students

to network and finding friends is easy.

12345

4. Sexual customs in U.S. are less conservative and many international graduate students would feel uneasy.

12345

5. Before I came to WVU, the transition program helped me to build a good relationship with my roommate $(\mathrm{s})$.

12345

\section{Financial Support}

1. The tuition waiver program for international graduate students at WVU is excellent.

12345

2. There are ample graduate assistantships for international graduate students at WVU.

12345

3. There' s a shortage of part-time jobs on campus for international graduate students at WVU.

12345

4. There are a good number of part-time jobs off campus for international graduate students at WVU. 12345

5. Most international graduate students barely have enough money to meet expenses.

12345 


\section{Spiritual Support}

1. Accesses to multiple faiths of the world are limited in and around WVU.

12345

2. The multiple faiths of U.S. make it confusing for international graduate students.

12345

3. I find it difficult to hear people criticize a certain faith in an open discussion.

12345

4. I would not know where to go if I need any spiritual guidance.

12345

5. I think people are very accepting at WVU of the multiple religions of the world.

12345

\section{Perceived Academic Success}

1. What's your description of the level of your knowledge learning through your program in WVU (Mid-Atlantic Land Grant Research University)?

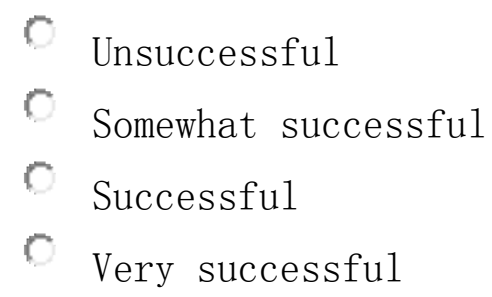


2. How would you like to describe your research skills?

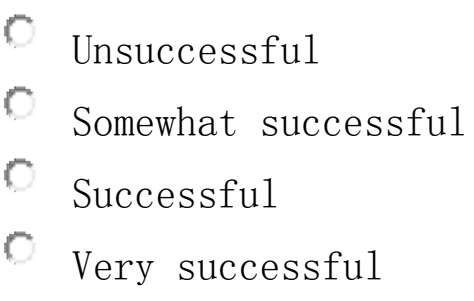

3. What do you think about your program in WVU to improve your employability?

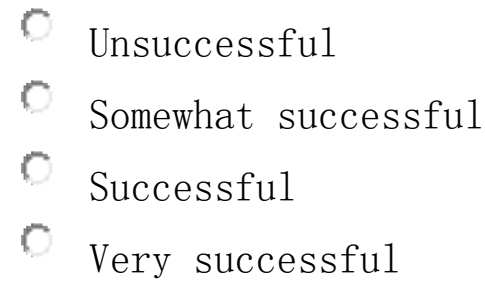

4. What's your opinion on your study in WVU related to your potential earning?

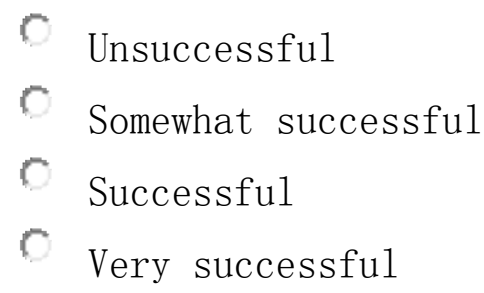

5. How would you like to describe your study in WVU related to your professional development?

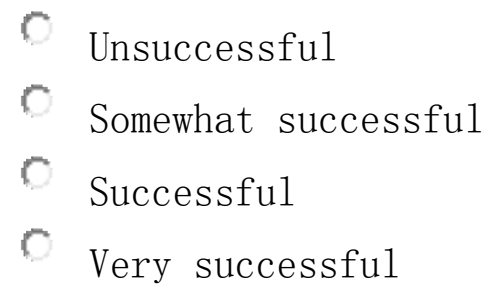

6. Compared to other international graduate students, what do you think about your study in WVU?

\section{Unsuccessful}




\section{Somewhat successful \\ Successful \\ V Very successful}




\section{Appendix D}

\section{Interview Questions}

$\underline{\text { Academic support }}$

1. Have you experienced any academic stress during your graduate study?

2. What is your perception of academic stress?

3. What types of academic issues have you encountered during your graduate study?

4. Have you received any support when you encountered these issues?

5. If yes, do you think these supports significantly affect the achievement of your academic success?

\section{$\underline{\text { Emotional support }}$}

6. Have you experienced any emotional problems during your graduate study?

7. What types of emotional problems have you encountered?

8. Have you received any support when you encountered these problems?

9. If yes, do you think these supports importantly influence the achievement of your academic success?

$\underline{\text { Financial support }}$

10. Have you experienced any financial problems during your graduate study?

11. What types of financial problems have you encountered?

12. Have you received nay support when you encountered these problems?

13. If yes, do you think these supports significantly affect the achievement of your academic success?

\section{$\underline{\text { Spiritual support }}$}

14. Have you experienced any religious dilemma during your graduate study? 
15. What types of religious problems have you encountered?

16. Have you received any support when you encountered these problems?

17. If yes, do you think these supports importantly influence the achievement of your academic success?

$\underline{\text { GPA }}$

18. Do you think GPA is important aspect of academic success for your graduate study?

19. Do you think that social support you receive will significantly affect your GPA?

20. If yes, how social support will affect your GPA? 


\section{Appendix E}

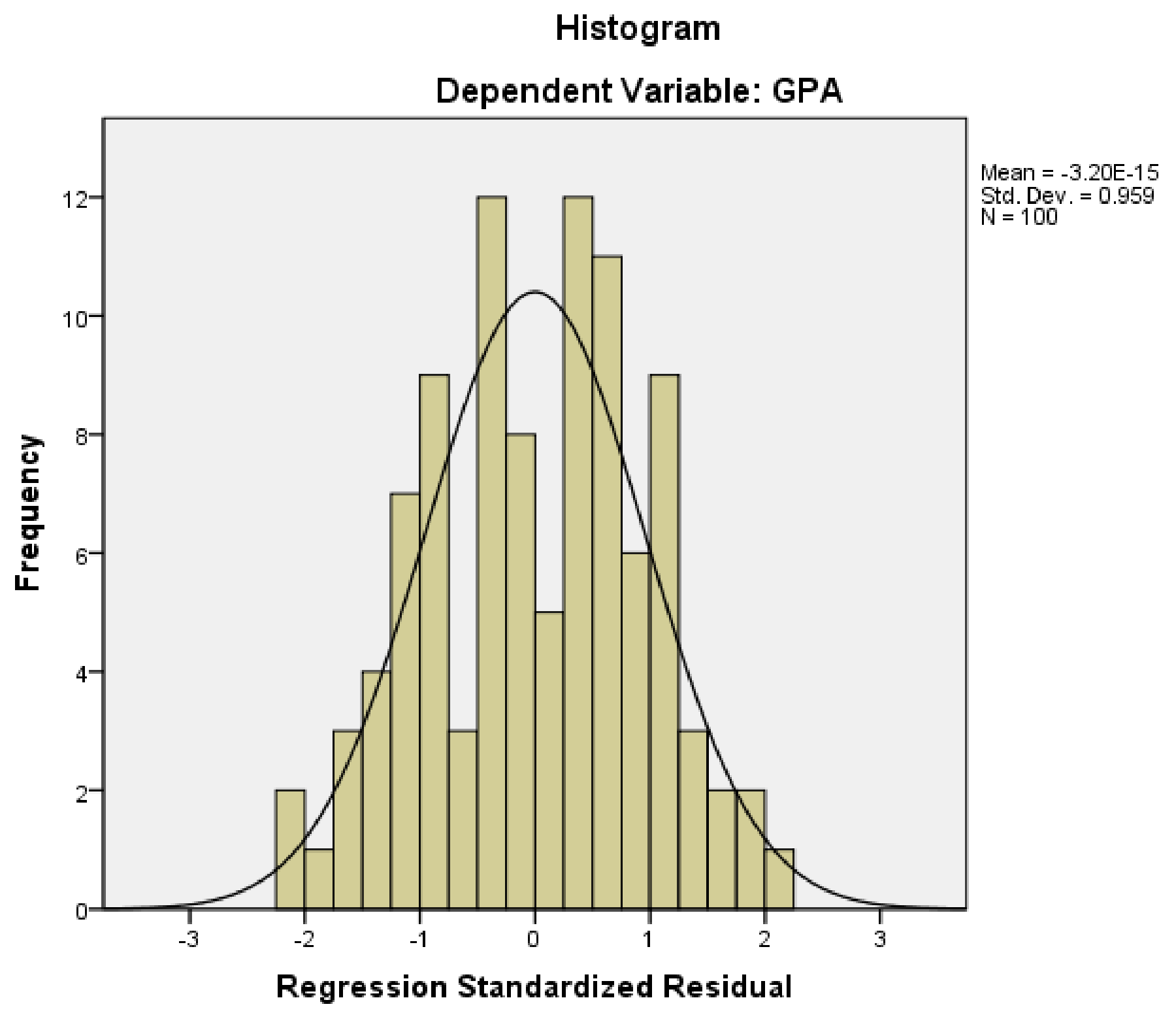




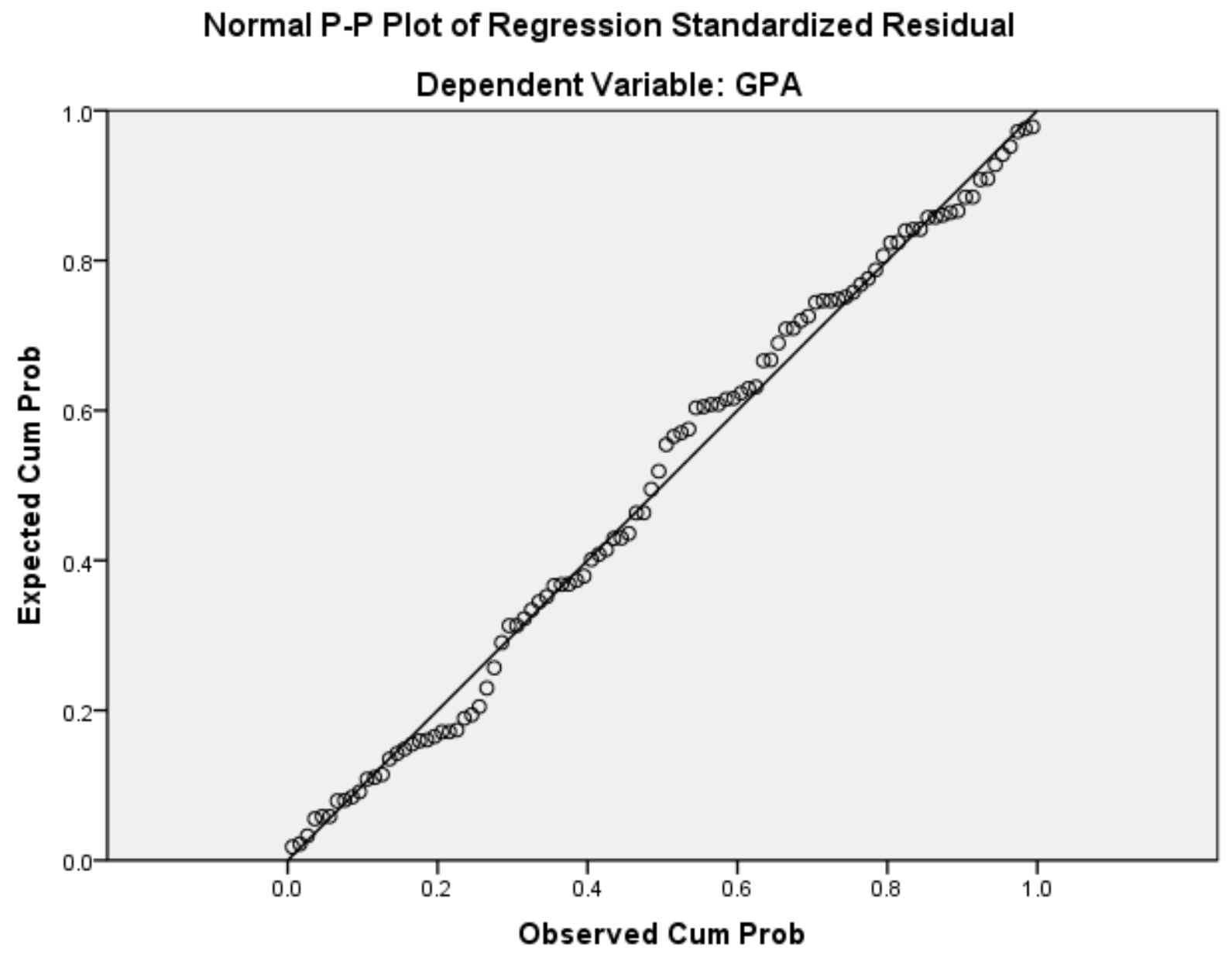




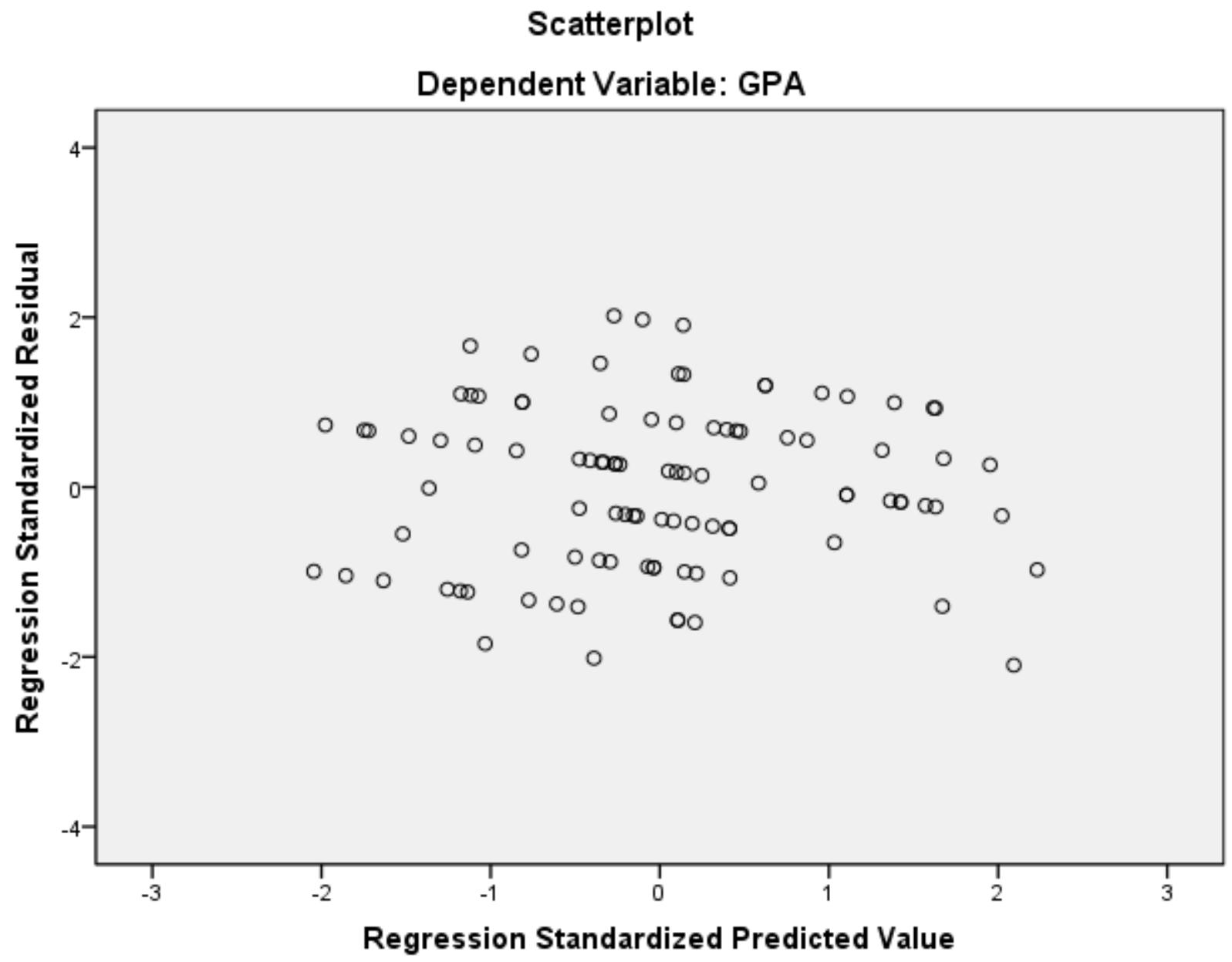




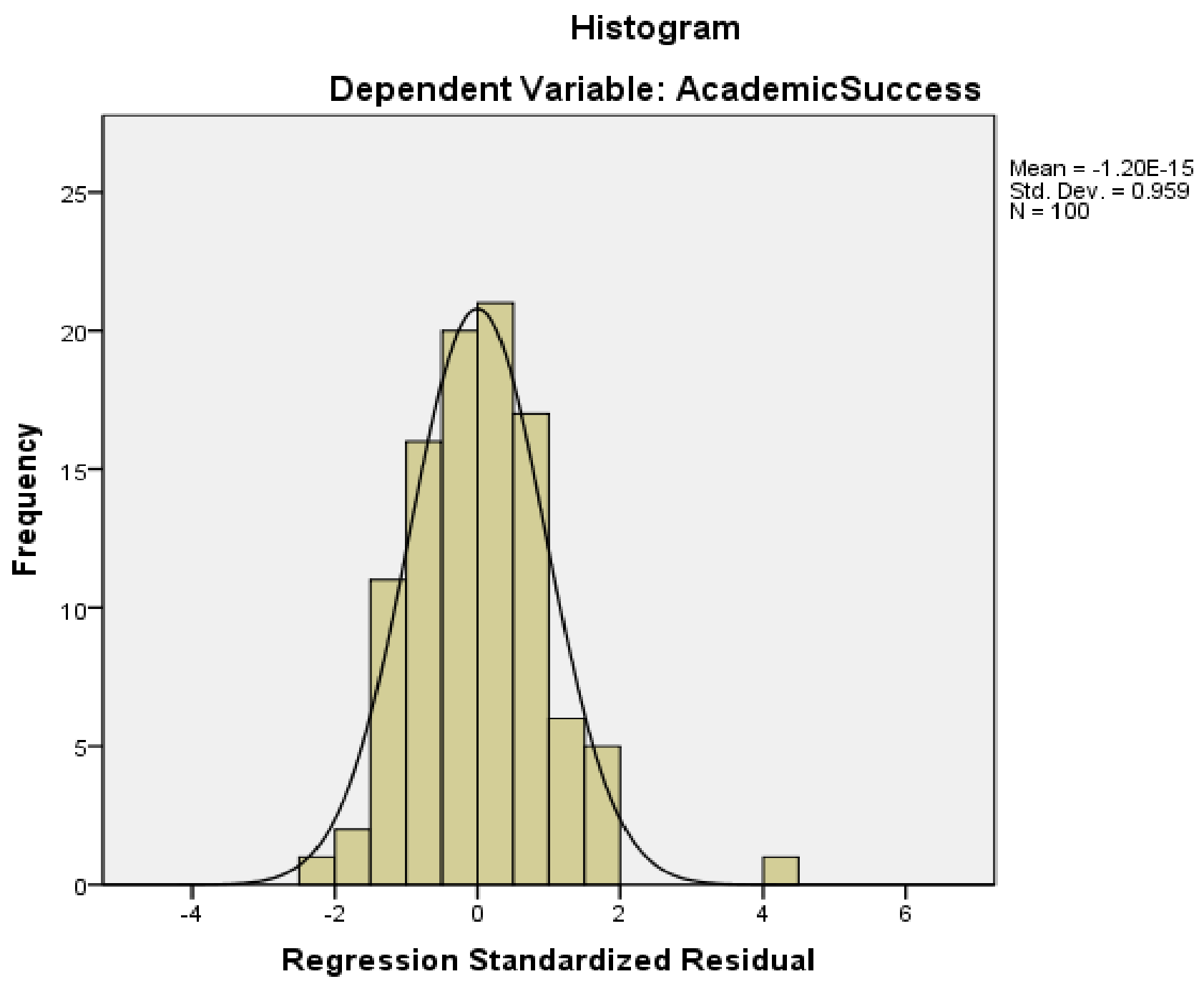




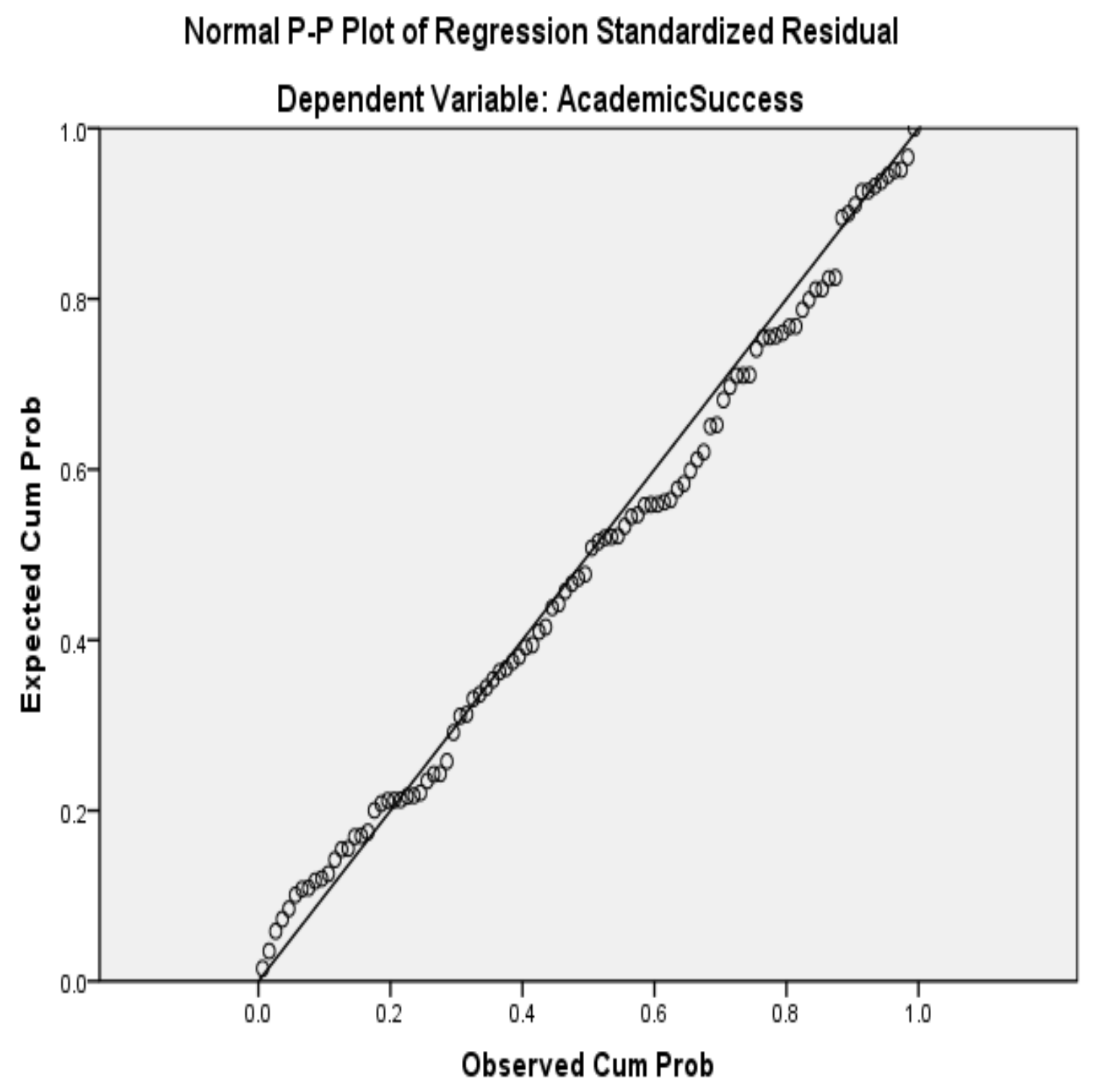




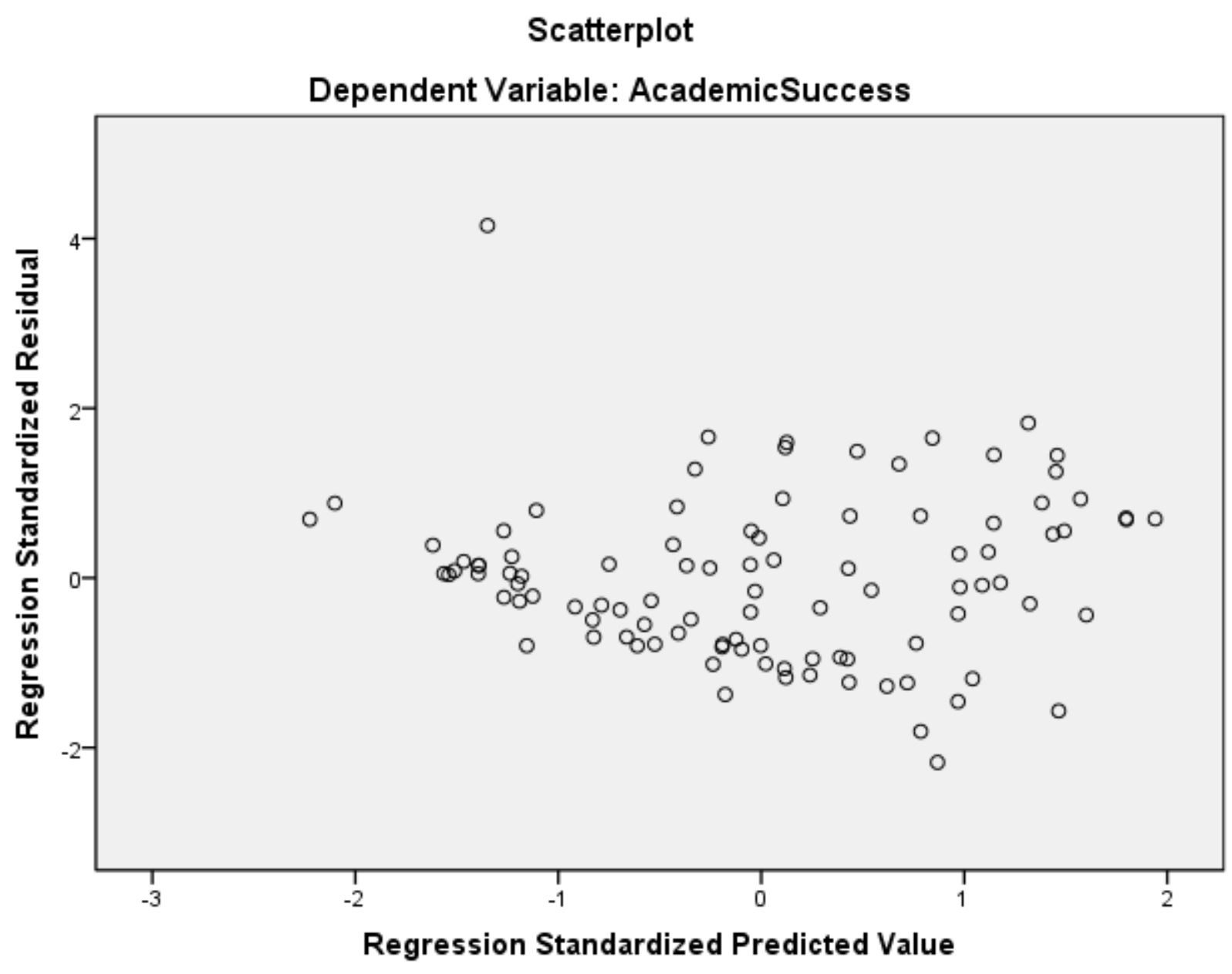




\section{Footnotes}

${ }^{1}$ Figure 1. Two points at which social support may interfere with the hypothesized casual link between stressful events and illness. Adapted from "Social Support Measurement and Intervention: A guide for health and social scientists" by Sheldon Cohen, Lynn G. Underwood and Benjamin H. Gottlieb, 2000, Copy right 2000 by Oxford ; New York : Oxford University Press. Adapted with permission. 